\title{
Stability studies of coal liquid products using high performance liquid chromatography
}

\author{
Lawrence P. Norcio \\ West Virginia University
}

Follow this and additional works at: https://researchrepository.wvu.edu/etd

\section{Recommended Citation}

Norcio, Lawrence P., "Stability studies of coal liquid products using high performance liquid chromatography" (1999). Graduate Theses, Dissertations, and Problem Reports. 2303.

https://researchrepository.wvu.edu/etd/2303

This Dissertation is protected by copyright and/or related rights. It has been brought to you by the The Research Repository @ WVU with permission from the rights-holder(s). You are free to use this Dissertation in any way that is permitted by the copyright and related rights legislation that applies to your use. For other uses you must obtain permission from the rights-holder(s) directly, unless additional rights are indicated by a Creative Commons license in the record and/ or on the work itself. This Dissertation has been accepted for inclusion in WVU Graduate Theses, Dissertations, and Problem Reports collection by an authorized administrator of The Research Repository @ WVU.

For more information, please contact researchrepository@mail.wvu.edu. 
Stability Studies of Coal Liquid Products Using

High Performance Liquid Chromatography

\author{
Lawrence P. Norcio \\ Dissertation submitted to the \\ College of Engineering and Mineral Resources \\ at West Virginia University \\ in partial fulfillment of the requirements \\ for the degree of
}
Doctor of Philosophy
in
Engineering

\author{
Edwin L. Kugler, Ph. D., Chair \\ John W. Zondlo, Ph. D. \\ Peter G. Stansberry, Ph. D. \\ Peter A. Hesbach, Ph. D. \\ Alfred H. Stiller, Ph. D.
}

Department of Chemical Engineering

Morgantown, West Virginia

1999

Keywords: Stability, Coal Liquids, HPLC, Molecular Weight Distribution Copyright 1999 Lawrence P. Norcio 


\author{
ABSTRACT \\ Stability Studies of Coal Liquid Products Using \\ High Performance Liquid Chromatography \\ Lawrence P. Norcio
}

The storage stability of coal liquids is of great importance if coal liquefaction products are to replace petroleum products. It has been reported that gum formation occurs in coal-derived liquids during storage to a level at which it becomes a handling problem. The aging of coal hydropyrolysis liquids has been studied. Coal liquid products were placed in preweighed sample vials and stored under light, in the presence of air, at different storage temperatures $\left(2,21\right.$, and $\left.32{ }^{\circ} \mathrm{C}\right)$. The weight of gum formed was determined at specified sampling times. High performance liquid chromatography (HPLC) was used to monitor the changes in hydrocarbon class composition with storage time. The changes in boiling point distribution were also determined using simulated distillation with an evaporative light-scattering detector.

Results show that the rate of gum formation is fastest at the highest storage temperature and slowest at the lowest storage temperature. The gum formed increased significantly at the early stage of storage and then remained constant with time. It was found that the saturate compound class remained constant with time at all storage conditions. However, both the polar and aromatic compound classes decreased significantly at the early stage of storage and then remained constant with time. The polar compounds were separated from the aromatic and saturate compound classes using solid phase extraction. No gum formation was observed for both the polar compounds and the aromatic-saturate mixture. Results from HPLC and simulated distillation method show no signs of changes in their chemical composition and boiling point distribution. It was concluded that the main reactants in gum formation in coal liquids are the aromatic and polar compound classes. Results from GC/MS and FTIR show that the polar compounds consist mainly of phenols. The affects of inert gas on the stability of coal liquids were determined by storing coal liquid samples in the presence of argon gas. It was then concluded that the presence of inert gas does not prevent the formation gum; instead, it slows down the rate of gum formation. A kinetic model was developed that describes the expermental data. 


\section{Dedicated Ta Hnei Li Chin with all my heart}




\section{ACKNOWLEDGMENTS}

I would like to thank Dr. Edwin L. Kugler, my research advisor, for his continuous guidance, support, and encouragement. Acknowledgement is also extended to Dr. Alfred H. Stiller, Dr. John W. Zondlo, Dr. Peter G. Stansberry, and Dr. Peter A. Hesbach, members of the research committee, for all their help and suggestions.

My appreciation is extended to Dr. Richard Turton and Dr. Dady Dadyburjor for their help with the reaction kinetics.

I would like to acknowledge partial support from the Consortium for Fossil Fuel Liquefaction Science, U.S. DOE Contract DE-FC 93PC93-53, and the National Research Center for Coal and Energy at West Virginia University.

I wish to thank Linda Rogers and Bonnie Helmick for secretarial work and Jim Hall for technical support.

I want to thank my friends, Zhu, Somjai, Swetha, Oguz, Bala, Adam, William, Venkat, David, Ramesh, and Yue who in a way helped me in this research. Thanks to Bhagat and Prasanna for the company during my overnight stays at the graduate room while typing this paper. Thanks to Huei Li for her help with some of the typing work and for her love and support. Finally, my sincerest thanks to my family and old friends for their affection, inspiration, encouragement, and unfailing support. 


\section{Table of Contents}

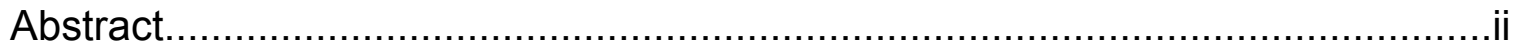

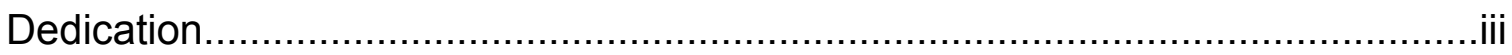

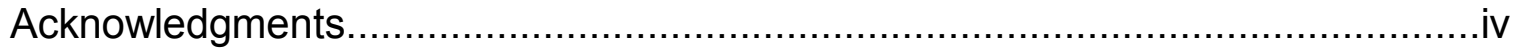

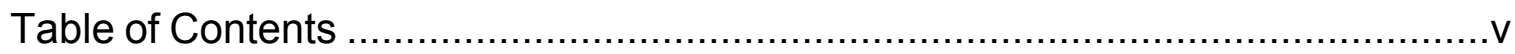

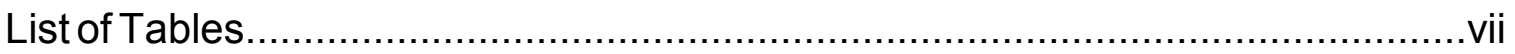

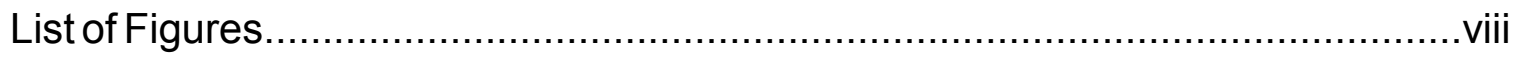

1.0 Introduction

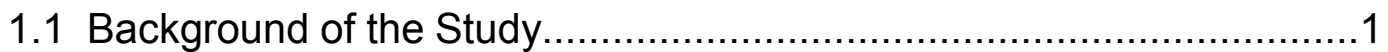

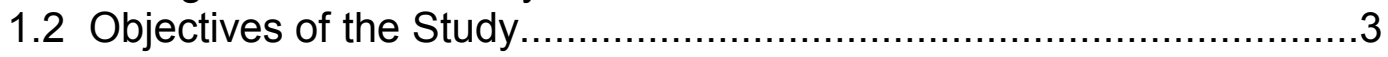

2.0 Review of Literature

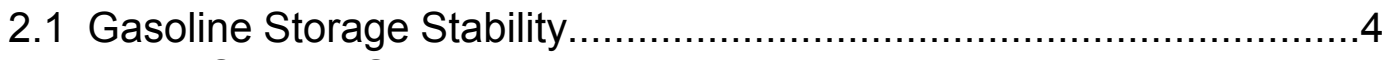

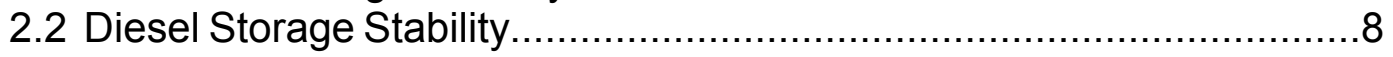

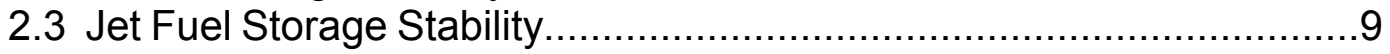

2.4 Fuel Oil Storage Stability ........................................................ 10

2.5 Coal Liquid Storage Stability.................................................. 12

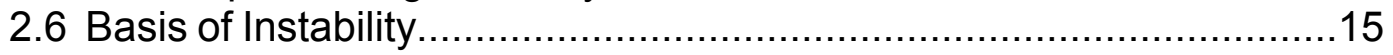

2.7 Filtrate Analysis Over Gum Analysis....................................... 16

3.0 Experimental Methods

3.1 Simulated Distillation by ELSD ................................................17

3.2 HPLC Hydrocarbon Compound Class Analysis................................21

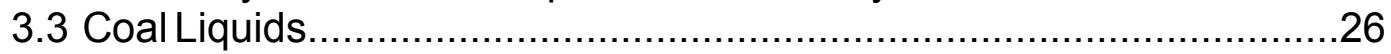

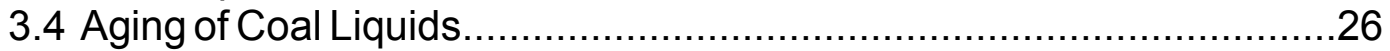

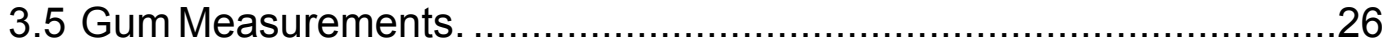

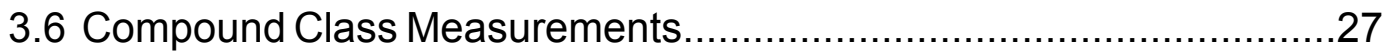

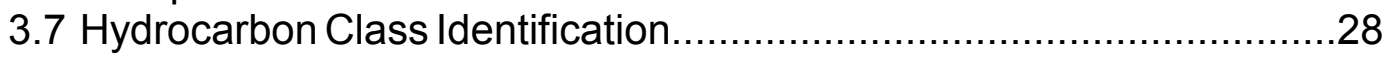


4.0 Results and Discussion

4.1 Molecular Weight Distribution Method ........................................29

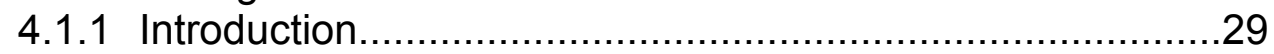

4.1.2 Determination of the MW Detection Limits........................30

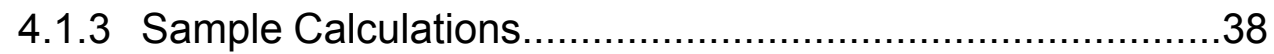

4.1.4 Results from MWD Test..............................................40

4.1.5 Conclusions for the MWD Method.................................. 42

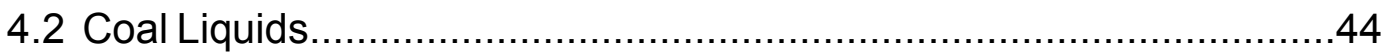

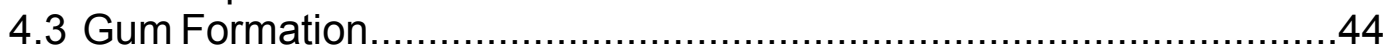

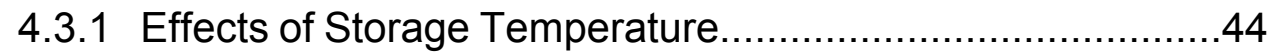

4.3.2 Gum Measurements.................................................53

4.4 HPLC Hydrocarbon Compound Class Analysis............................... 54

4.4.1 Compound Class Separations.........................................54

4.4.2 Changes in Coal Liquid Composition...............................59

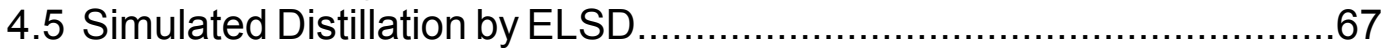

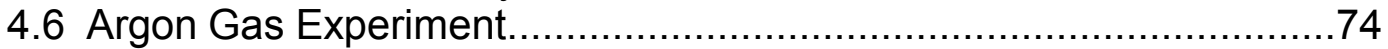

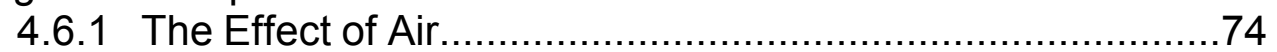

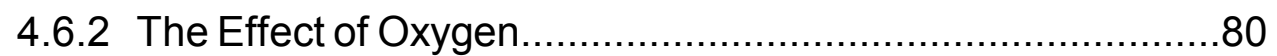

4.7 Main Reactants in Gum Formation................................................ 81

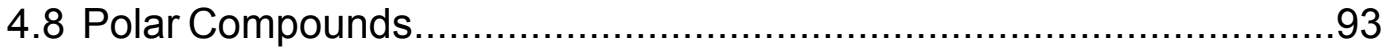

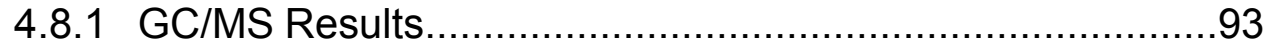

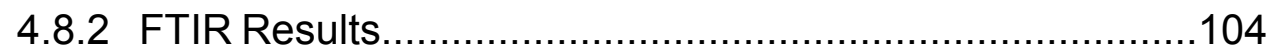

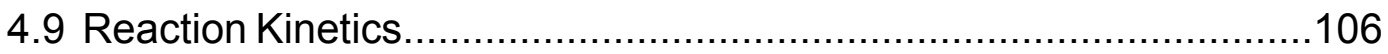

4.9.1 Overview of the Degradation Reaction............................106

4.9.2 The Proposed Kinetic Model........................................108

4.8.3 Developing the Kinetic Model.......................................109

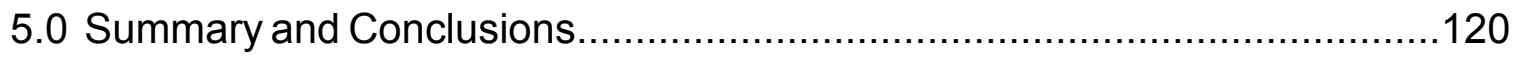

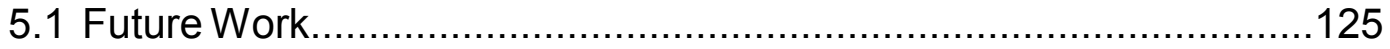

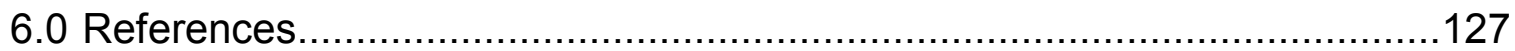

Appendix $1 \quad$ Procedure Used in Coal Liquefaction...............................134

Appendix $2 \quad$ Sample Calculation of the Total Conversion......................137

Appendix 3 Three-Dimensional UV Spectrum Plot of the Aromatic Standards Used.........................................139

Appendix $4 \quad$ Kinetic Data for Coal Pyrolysis Liquids..............................141

Appendix $5 \quad$ Data for Arrhenius Plots..............................................145

Appendix $6 \quad$ GC/MS Methods Used for the Analysis.........................147

Appendix $7 \quad$ Conditions Used in HPLC Analysis.................................150

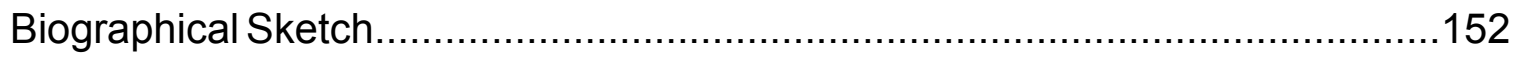




\section{List of Tables}

Table 3.1 Columns and Solvent Compositions Used for Hydrocarbon Compound Class Analysis. Solvent Flowrate is at $1.2 \mathrm{~mL} / \mathrm{min}$

Table 4.1 The Alkane Compounds Used in Obtaining the Data in Figure 4.3

Table 4.2 The Aromatic Compounds Used in Obtaining the Data in Figure 4.4

Table 4.3 The MW Detection Limits for the Alkane and Aromatic

Series.

Table 4.4 Results from Testing the Standard Mixtures at $40^{\circ} \mathrm{C}$

Table 4.5 Initial Rates of Gum Formation of Coal Liquids at

Different Storage Temperatures.

Table 4.6 Retention Times of Pure Standards for the HPLC

Hydrocarbon Compound Class Analysis.

Table 4.7 Typical Boiling Point Distribution Range of a Fresh Coal Liquid Sample....

Table 4.8 Results from the Hydrocarbon Compound Class Analysis and Weight Percent of Gum Produced in the Absence of Air

Table 4.9 List of Polar Compounds Identified by GC/MS ...........................103

Table 4.10 Reaction Rate Constants Obtained from Equation 4.7 at Different Storage Temperatures.

Table 4.11 Equilibrium Constants Obtained from Equation 4.13 at Different Storage Temperature. 


\section{List of Figures}

Figure 3.1 High Performance Liquid Chromatography (HPLC) Used

in the Stability Analysis of Coal Pyrolysis Liquids.

Figure 3.2 The Boiling Point Distribution Setup .........................................19

Figure 3.3 Block Diagram of the Evaporative Light-Scattering Detector.........20

Figure 3.4 The Arrangement of the Three Columns in the HPLC System

Figure 4.1 Responses of Different Types of Compounds with

Different MW

Figure 4.2 Responses of $\mathrm{C}_{22}$ in Different Solvents.

Figure 4.3 Responses of a Series of n-Alkanes as a Function

of MW (Arrows Represent the MW Detection LImits)

Figure 4.4 Responses of a Series of Aromatics as a Function of MW (Arrows Represent the MW Detection LImits).

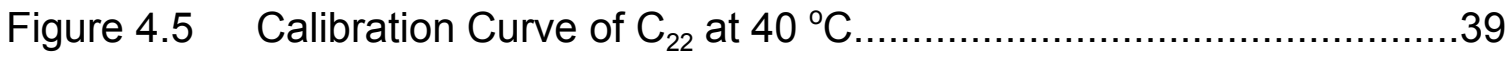

Figure 4.6 MW Detection Limit as a Function of Detector

Temperature.

Figure 4.7 (a) Picture of Fresh Coal Liquid Sample;

(b) Picture of Aged Coal Liquid Sample

Stored at $21^{\circ} \mathrm{C}$ for 72 hours.

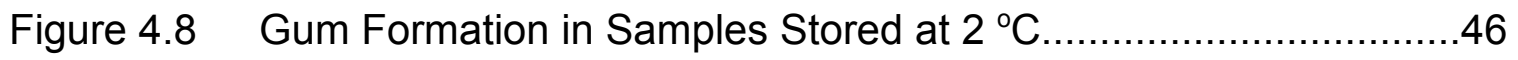

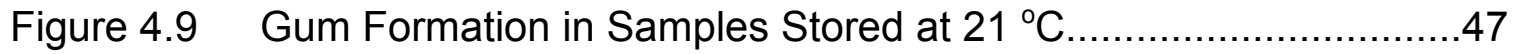

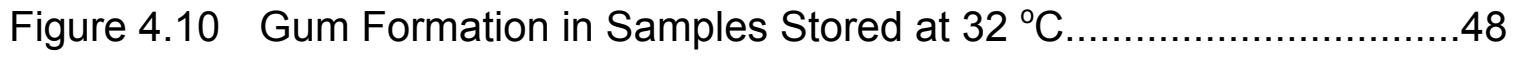

Figure 4.11 The Effect of Temperature on the Rate of Gum Formation.

Figure 4.12 A Three-Dimensional UV Spectrum Plot of

a Fresh Coal Liquid Sample. 
Figure 4.13 Absorbance Plot of the Same Data Shown in Figure 4.12

for 2-, 3-, and 4-Ring Aromatics at $259 \mathrm{~nm}$.

Figure 4.14 ELSD Chromatogram of a Fresh Coal Liquid Sample....................58

Figure 4.15 Changes in Weight Percentage Saturate Compound

Class Stored at $2,21,32^{\circ} \mathrm{C}$ (Data Obtained from ELSD)

Fiigure 4.16 Changes in Weight Percentage Aromatic Compound

Classs Stored at $2,21,32^{\circ} \mathrm{C}$ (Data Obtained from ELSD)

Figure 4.17 Changes in Weight Percentage Polar Compound Class

Stored at 2, $21,32{ }^{\circ} \mathrm{C}$ (Data Obtained from ELSD).

Figure 4.18 ELSD Chromatogram of Fresh and Aged (72 hours) Coal

Liquids Showing the Changes in Saturates and

1-Ring Aromatics.

Figure 4.19 ELSD Chromatogram of Fresh and Aged (72 hours) Coal

Liquids Showing the Changes in Multi-Ring Aromatics.

Figure 4.20 ELSD Chromatogram of Fresh and Aged (72 hours) Coal Liquids Showing the Changes in Polar Compounds.

Figure 4.21 A Typical ELSD Chromatogram Obtained from

the Simulated Distillation Method.

Figure 4.22 Changes in Weight Percent Coal Liquid from ELSD

with Boiling Point Between $315^{\circ} \mathrm{C}$ and $380^{\circ} \mathrm{C}$ Stored at

Different Temperatures

Figure 4.23 Changes in Weight Percent Coal Liquid from ELSD

with Boiling Point Between $380{ }^{\circ} \mathrm{C}$ and $435^{\circ} \mathrm{C}$ Stored at

Different Temperatures.

Figure 4.24 Changes in Weight Percent Coal Liquid from ELSD

with Boiling Point Between $435^{\circ} \mathrm{C}$ and $482{ }^{\circ} \mathrm{C}$ Stored at

Different Temperatures.

Figure 4.25 Changes in Weight Percent Coal Liquid from ELSD

with Boiling Point Greater than $482{ }^{\circ} \mathrm{C}$ Stored at

Different Temperatures.

Figure 4.26 Comparison of Weight Percent Saturates Stored at $21^{\circ} \mathrm{C}$ With and Without Air. .76 
Figure 4.27 Comparison of Weight Percent Aromatics Stored at $21^{\circ} \mathrm{C}$

With and Without Air.

Figure 4.28 Comparison of Weight Percent Polars Stored at $21^{\circ} \mathrm{C}$

With and Without Air.

Figure 4.29 Comparison of Weight Percent Gum Formed when

Stored at $21{ }^{\circ} \mathrm{C}$ With and Without Air....

Figure 4.30 Solid Phase Extraction Tube Separating Polar

Compounds from Saturate-and-Aromatic Compounds.

Figure 4.31 Fresh Saturates-and-Aromatics, and Polar Compounds

After Separation by Solid Phase Extraction.

Figure 4.32 Changes in Saturates-and-Aromatics Stored at $2,21,32^{\circ} \mathrm{C}$

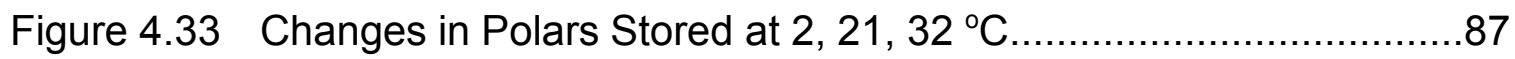

Figure 4.34 Changes in Boiling Point Distribution in $315^{\circ} \mathrm{C}>\mathrm{BP}>380^{\circ} \mathrm{C}$ for Saturates-and-Aromatics.

Figure 4.35 Changes in Boiling Point Distribution in $315^{\circ} \mathrm{C}>\mathrm{BP}>380^{\circ} \mathrm{C}$ for Polar Compounds

Figure 4.36 Changes in Boiling Point Distribution in $380^{\circ} \mathrm{C}>\mathrm{BP}>435^{\circ} \mathrm{C}$ for Saturates-and-Aromatics.

Figure 4.37 Changes in Boiling Point Distribution in $380^{\circ} \mathrm{C}>\mathrm{BP}>435^{\circ} \mathrm{C}$ for Polar Compounds

Figure 4.38 Changes in Boiling Point Distribution in $435^{\circ} \mathrm{C}>\mathrm{BP}>482^{\circ} \mathrm{C}$ for Saturates-and-Aromatics.

Figure 4.39 Changes in Boiling Point Distribution in $435^{\circ} \mathrm{C}>\mathrm{BP}>482^{\circ} \mathrm{C}$ for Polar Compounds.

Figure 4.40 Changes in Boiling Point Distribution in BP $>482{ }^{\circ} \mathrm{C}$ for Saturates-and-Aromatics.

Figure 4.41 Changes in Boiling Point Distribution in $\mathrm{BP}>482{ }^{\circ} \mathrm{C}$ for Polar Compounds. 
Figure 4.42 GC/MS Total Ion Chromatogram of Polar Compounds

(Masses of Prominent Peaks are Shown)

Figure 4.43 (a) Mass Spectrum of Sample,

(b) Possible Match of Phenol from MS Library

Figure 4.44 (a) Mass Spectrum of Sample,

(b) Possible Match of 2 - methyl phenol from MS Library.

Figure 4.45 (a) Mass Spectrum of Sample,

(b) Possible Match of 3 - methyl phenol from MS Library

Figure 4.46 (a) Mass Spectrum of Sample,

(b) Possible Match of 2, 3 - dimethyl phenol from MS Library.

Figure 4.47 (a) Mass Spectrum of Sample,

(b) Possible Match of 2 - ethyl phenol from MS Library.... 100

Figure 4.48 (a) Mass Spectrum of Sample,

(b) Possible Match of 2, 4 - dimethyl phenol from MS Library

Figure 4.49 (a) Mass Spectrum of Sample,

(b) Possible Match of 3 - ethyl - 5 - methyl phenol from MS Library.

Figure 4.50 Infrared Spectrum of the Polar Compound Class. 105

Figure 4.51 Plot of the Rate of Gum Formation from Experimental

Data versus Kinetic Model at $\mathrm{T}=2^{\circ} \mathrm{C}$

Figure 4.52 Plot of the Rate of Gum Formation from Experimental Data versus Kinetic Model at $\mathrm{T}=21^{\circ} \mathrm{C}$

Figure 4.53 Plot of the Rate of Gum Formation from Experimental Data versus Kinetic Model at $\mathrm{T}=32^{\circ} \mathrm{C}$

Figure 4.54 Plot of Gum Formation from Experimental Data versus Data from the Model at 2,21 , and $32^{\circ} \mathrm{C}$

Figure 4.55 Arrhenius Plots for the Forward and Backward Reactions of Coal Pyrolysis Liquids Coal Liquids 


\subsection{INTRODUCTION}

\subsection{Background of the Study}

World petroleum reserves are being rapidly consumed at a rapid rate $[1,2$, $3,4,5]$. This situation led to research on alternative liquid fuel sources as a substitute for petroleum. One of these alternative new sources is liquid fuel from coal.

All fuels have chemical instability problems. Fuel stability is defined as the general resistance to change $[1,9]$. A measure of stability is the ability of fuel to remain in storage over extended periods of time without significant deterioration [1,

$9,11]$. Problems associated with storage instability of fuels include formation of sediments and gummy materials $[1,2,6,7,8,9,10]$, production of coke or fouling materials $[1,9]$, color changes $[1,9,10]$, deterioration of their physical and chemical characteristics, composition, and quality [1,9]. The formation of sediments may result in fuel filter and pipe lines clogging $[1,9]$. Moreover, these deposits attract and bind dirt, rust and other corrosive products found in fuel systems [9]. Microorganisms may also grow given a suitable environment leading to microbial contamination, deterioration and corrosion [9].

Gum content measures the quality of the fuel and may be used as a guide for future behavior of synthetic fuels (synfuels) in storage [9]. Insoluble gum will clog screen and engine filters and will eventually cause fuel injectors to become inoperable $[1,9]$. Coking and fouling materials in fuel will cause engine operating 
difficulties [9]. Engine noise, excessive smoke, loss of power, poor fuel economy, degraded emissions, and poor drivability have been attributed to coking [9].

A color change into darker or deeper color than the original is an indication of instability and is not desirable for marketing purposes [1,9]. Clear, light colored fuel products are preferable in the market.

The changes in physical properties like viscosity and density tend to reduce the quality of synfuels and lead to the off specification of the product [9]. In addition, degraded fuel tends to increase maintenance problems and costs, equipment vulnerability and decreased reliability. Chemical changes will also reduce the quality of fuels. Formation of peroxides or hydroperoxides will damage elastometric gasket materials in the engine combustion system $[9,10]$. Changes in combustion properties may be troublesome in engine performance and combustion efficiency [9].

There has been much research on coal-derived liquids, but most of these studies focused on the upstream processing technology. Only a small number of studies have been reported regarding the analysis of the stability of coal liquid products. Thus, stability studies on coal liquids are a vital necessity. This will provide information essential in the assessment of the storage quality of the product. Understanding stability of coal liquids is important before undertaking rational ways of preventing instability problems. 


\subsection{Objectives of the Study}

The main objectives of this study are to monitor the changes of coal liquid composition with time using high performance liquid chromatography (HPLC), and to develop a kinetic model that will describe the rate of gum formation. This will involve the observation of coal liquid products with time, the development of a correlation between the changes in coal liquid composition with the rate of gum formation, and the separation and quantification of coal liquid components using HPLC. 


\subsection{REVIEW OF LITERATURE}

Liquid fuels from alternative sources could alleviate fuel shortages caused by the decreasing supply of petroleum. However, studies on stability characteristics of alternative fuels are scarce. As a result, stability guidelines are based on previous work with petroleum stability studies [1, 12].

\subsection{Gasoline Storage Stability}

Gasoline is a mixture of hydrocarbons which contains small amounts of sulfur and nitrogen compounds [18]. These compounds react with oxygen in the air and with one another during storage to produce high molecular weight substances called gums $[1,2,7,8,9,10,18]$.

Bowden [19] developed storage stability data on available fuels to serve as reference data. He used ten samples each of unleaded gasoline, leaded gasoline, No. 2 diesel fuel, and JP-4 jet fuel. He evaluated each sample for physical properties, then stored ten $280 \mathrm{~mL}$ portions of each sample in $32 \mathrm{oz}$. amber glass bottles at $43{ }^{\circ} \mathrm{C}$. The bottles were aerated every four weeks. Two bottles of each sample were removed from storage and analyzed by NMR, UV spectroscopy and elemental analysis for soluble and insoluble gum precipitate after 4, 8, 16 and 32 weeks of storage. Deterioration of sample was estimated by the increase of gum formation.

A similar method was done by Nixon et al. [20] when they studied the effects of a number of different alkyl phenols on various gasoline properties including 
storage stability. They stored gasoline samples in amber bottles at either $60^{\circ} \mathrm{C}, 70$ ${ }^{\circ} \mathrm{C}$ or $43{ }^{\circ} \mathrm{C}$ in an atmosphere of oxygen.

Schwartz and Ward [21] performed storage tests at $43^{\circ} \mathrm{C}$ with mixtures of sulfur compounds and reactive hydrocarbons in $n$-heptane. The bottles were opened and the air replenished periodically during storage. They found that periodic aeration resulted in the formation of larger amounts of gum compared to sealed storage. Elemental analysis of the gums showed that they were similar to those from full-boiling range fuels.

In another experiment, Schwartz and Ward [22] stored a series of gasolines and gasoline blending stocks at $43^{\circ} \mathrm{C}$ in sealed glass bottles and in glass bottles aerated periodically, and in stainless steel bombs aged at $93{ }^{\circ} \mathrm{C}$ and at $121^{\circ} \mathrm{C}$. They found that maintaining excess oxygen on storage increased gum formation compared to sealed storage. The bomb tests at $93^{\circ} \mathrm{C}$ and $121^{\circ} \mathrm{C}$ correlated with the $43^{\circ} \mathrm{C}$ storage results.

A standard test method for fuel storage stability at $43^{\circ} \mathrm{C}$ is available (ASTM D4625) [15]. Filtered fuel samples are aged in borosilicate glass containers at 43 ${ }^{\circ} \mathrm{C}$ for periods of $0,4,8,12,18$, and 24 weeks. After aging for the selected period of time, the sample is removed from storage and allowed to cool to $21^{\circ} \mathrm{C}$ to $27^{\circ} \mathrm{C}$ in a dark enviroment. Cooling would take 4 to 24 hours. After cooling, the sample is filtered through a tared Gooch crucible containing glass-fiber filters. The bottle containers are rinsed with isooctane to remove all traces of fuel from the bottle. The rinsings are also filtered through the Gooch crucible. The crucible is dried in the oven for 4 hours, cooled, and weighed. This gives the weight of filterable 
insolubles. The adherent gum in the bottle container was dissolved by the adherent insoluble solvent (mixture of equal amounts of acetone, methanol and toluene). The washings are placed in a preweighed beaker. The beaker is dried at $160^{\circ} \mathrm{C}$, cooled, and weighed. This gives the weight of adherent insolubles. The total insolubles are calculated as

$$
T=\frac{(A+B)}{4}
$$

where $T=$ total insolubles $(\mathrm{mg} / 100 \mathrm{~mL}), A=$ weight of filterable insolubles $(\mathrm{mg})$, and $B=$ weight of adherent insolubles $(\mathrm{mg})$. It has been shown that aging fuel at $43^{\circ} \mathrm{C}$ is equivalent to four-fold acceleration of the degradation at ambient temperature of $21^{\circ} \mathrm{C}[15,17]$. Depending on fuel composition, a week of $43^{\circ} \mathrm{C}$ storage is approximately equivalent to one month storage at ambient temperatures [15].

Other accelerated methods for deterioration of fuel involved storing them at even higher temperatures than $43^{\circ} \mathrm{C}$. Kawahara [23] produced the first gum by aging naphtha in steel drums at $60^{\circ} \mathrm{C}$ for 12 weeks and produced the second gum by aging naphtha in steel drums for two years at ambient conditions. The gums were analyzed and both gums contained thioether, dialkyl peroxide, and other groups, as well as ester, carbonyl, acid, hydroperoxide, and hydroxyl groups.

Schwartz et al. [24] described a rapid test for predicting gasoline stability. They stored a gasoline sample in a sealed glass bottle at $93{ }^{\circ} \mathrm{C}$ for 16 hours. The amount of gum formed, and the amount of oxygen consumed by the gasoline was used to predict the amount of gum and inorganic residue that would be formed in 
$43{ }^{\circ} \mathrm{C}$ storage. The storage performance of motor gasoline for extended periods could be estimated at any temperature up to $43^{\circ} \mathrm{C}$.

Gums have been characterized using spectrophotometric, chromatographic, NMR, and other methods. Bassler and Smith [25] studied gum formation in gasoline and in pure alkane and alkenes by accelerated storage using irradiation by ultraviolet (UV) light. Infrared analysis of the gum showed the presence of carboxyl, hydroxyl, carbonyl, and ester functional groups.

Schwartz and Ward $[26,27]$ used a spectrophotometric method to detect insoluble gums. This method measured the darkening of the ageing fuel by using a colorimeter. A decline in light transmission with time suggested that the fuel is becoming darker and forming significant amounts of gum.

In another study, Schwartz and Ward [28] used UV irradiation to accelerate gum formation. Aromatic, olefinic and paraffin-naphthene hydrocarbons were separated by silica gel chromatography from each of the fuel samples and distilled to produce distillation fractions of each silica gel portion. The gum content before and after storage of each fraction was determined and the most unstable fractions were included in the olefinic and aromatic portions.

Schwartz and Ward [29] also studied the changes in aromatics, sulfur and nitrogen contents of fresh and aged gasoline samples using UV irradiation to accelerate gum formation. It was found that elemental sulfur, hydrogen sulfide, mercaptans, sulfides and disulfides reacted in gasoline aged by UV irradiation. Other types of sulfur compounds did not react and they concluded that sulfur compounds are not essential for gum formation. Similarly, they also concluded that 
nitrogen compounds are not essential for gum formation. The only nitrogen compounds involved in gum formation were pyridines, quinolines, anilines, and amines. However, they found that aromatics reacted to form gums and the decreasing order of reactivity was: alkyl benzenes, tetralins, indans, and naphthalenes.

Allbright et al. [30] used a radioactive tracer technique to determine the types of compounds that cause gum formation in gasoline during storage. They labelled selected organic compounds with tritium. After storage at $43^{\circ} \mathrm{C}$ for 16 weeks, the gums were analyzed. The amount of test compound converted to gum was determined. The compounds listed in order of decreasing percentage of each in the gum are: mono-olefins, alkyl benzenes, sulfur compounds, polycyclic hydrocarbons, nitrogen compounds and diolefins.

\subsection{Diesel Storage Stability}

Specification of diesel fuels may have many and varying combinations of physical and chemical properties. Color of diesel fuel is measured by the ASTM color test (ASTM D1500) [14]. The sample is placed in a test container and compared with colored glass disks ranging from 0.5 to 8.0 . The determination is made when the glass disk matches the color of the sample. In general, a highly degraded product will have the darkest color.

The application of light scattering to diesel fuel stability studies has been reported. This technique examines the growth of small particles which are precursors to deposit formation. It is based on the principle that as fuel ages, particles will grow in size and scatter light $[9,18]$. 
Christian et al. [31, 32] stored diesel fuels in borosilicate and soft glass bottles at $54{ }^{\circ} \mathrm{C}$ and $98^{\circ} \mathrm{C}$. Degradation was measured by the change in light transmission and light scattering and by the amount of insoluble residue and soluble gum formed. In addition, they found that diesel fuel stored in borosilicate glass bottles deteriorated much more rapidly than the same fuel stored in soft glass bottles. Their experiment showed that soft glass inhibited fuel degradation while borosilicate glass was essentially inert toward the fuel. They have noted that the effect of soft glass varied with the fuel. It may improve stability of some fuels but has almost no effect on other fuels. Therefore, using soft glass bottles as storage containers could give misleading results in assessing the stability of fuels.

\subsection{Jet Fuel Storage Stability}

The influence of a few nitrogen compounds on storage stability of jet fuels was studied by Worstell and Daniel [33] using the microscope cover slip method. The four classes of heterocyclic nitrogen compounds that they evaluated were: pyrroles, indoles, pyridines, and quinolines. The nitrogen compounds were dissolved in tetrahydrofuran (THF) to the desired nitrogen concentration and added to the jet fuel samples. The mixtures were stored in an oven at $120{ }^{\circ} \mathrm{C}$ with a microscope cover slip in each bottle. After 168 hours, the bottles were taken out from the oven and the cover slips were removed, dried, and weighed. They found that pyrrole, indole, pyridine, and quinoline produced a very small increase in gum formation compared to the jet fuel sample alone. Moreover, they found that steric hindrance at the nitrogen atom reduced effectiveness in promoting gum formation. 
The effect of sulfur-containing compounds on the storage stability of Jet A fuel was investigated by Heneman [34] using the microscope cover slip method developed by Worstell [33]. It was found that thiols and thiophene derivatives increased the rate of gum formation compared to that of pure jet fuel. Alkyl sulfides and disulfides decreased the rate of gum formation. The inhibiting mechanism of alkyl sulfides appeared to be a result of sulfur's reactivity with intermediate soluble precursors.

Nixon et al. [35] measured total and insoluble gum of jet fuels stored in steel drums for four years. The straight run fuel was the most stable, thermally cracked fuel was the least stable and catalytically cracked fuel was intermediate in stability. A small apparatus for measuring filter ability of jet fuels was developed in their laboratory. They found no correlation between filter-plugging tendency and insoluble gum content of a fuel. Using the electron microscope, it was found that the filter behavior of insoluble gums is related to their character. For example, an amorphous type deposit rapidly plugged the filter, while a crystalline type deposit did not.

\subsection{Fuel Oil Storage Stability}

Gyrath et al. [36] performed a one day stability test of fuel oil. They placed one liter of oil in a glass bottle along with several steel strips. The bottle was purged with oxygen, sealed, and placed in an oven at $100^{\circ} \mathrm{C}$ for 24 hours. At the end of the test, the oil was cooled and filtered, and the deposit on the filter was dried and weighed. The results of the one day test were correlated with the results 
of storage tests in which oil samples were stored for 6 months at $29^{\circ} \mathrm{C}$ in 30 gallon steel drums or in glass bottles containing steel strips.

Konrad et al. [37] developed an accelerated filter plugging test for measuring the storage stability of distillate fuels. The test oil was placed in a tin-plated steel can and heated for 16 hours where upon the oil temperature started at $23^{\circ} \mathrm{C}$ and reached $112^{\circ} \mathrm{C}$ by 16 hours. The oil was then cooled and filtered through a woven felt filter. Pressure drop across the filter was measured and the sediment deposited on the filter was weighed. Both parameters were related to the stability of the fuel. Loveland et al. [38] stored fuel oils and electrical oils in glass bottles at $43^{\circ} \mathrm{C}$. The aged and fresh fuels were examined by light scattering methods. However, when large quantities of sludge were formed, leading to aggregation of small particles into large particles, the light scattering method was no longer valid. They suggested that light scattering would be a good application for gum determination after short aging times only.

Milsom and Rescorla [39] studied the storage stability of several fuel oils stored in glass bottles at four different temperatures--- $43^{\circ} \mathrm{C}, 65^{\circ} \mathrm{C}, 82^{\circ} \mathrm{C}$, and 100 ${ }^{\circ} \mathrm{C}$. The bottles were opened and aerated each day. The amount of insolubles formed after each storage period was measured. Correlations and equations were developed by which time of storage and temperature up to $100{ }^{\circ} \mathrm{C}$ could be converted to equivalent time of storage at any other temperature. 
Walker and Stanton [40] used microscopic examination of oil to estimate the storage stability of the oil. The number of particles in the liquid oil were measured with a light microscope. The increase in the number of particles in the oil after storage was correlated with the storage stability of the oil.

\subsection{Coal Liquid Storage Stability}

With petroleum as a nonrenewable natural resource, synfuels from coal, for example, become of increasing importance. However, coal-derived liquids are complex in that it is difficult to define the mechanisms, reactive compounds and methods to stabilize these materials [18].

Brown and Karn [41] performed stability studies of raw coal liquids obtained from the one-half-ton-per-day process development unit at Pittsburgh Energy Technology Center. The samples were obtained from process runs operated at 27.6 Mpa pressure and $450{ }^{\circ} \mathrm{C}$. The liquid samples were aged under various combinations of temperature, gaseous environment (nitrogen, air or oxygen), mechanical agitation, and the absence or presence of ambient light. Degradation was measured primarily by viscosity. The viscosity increased with aging of all samples. The rate of viscosity increase of the samples stored under oxygencontaining gases was faster than those stored under inert (nitrogen) environment. Moreover, the rate of degradation is faster at higher storage temperatures. They concluded that oxygen has a deleterious effect on the stability of coal liquids. Solvent analysis showed that the samples aged under oxygen had a significant increase in the amount of benzene- insoluble material, a decrease in oil components and a relatively constant amount of asphaltenes. Samples aged under 
nitrogen showed very little change. Solvent analysis of fresh and oxygen-aged samples showed a decrease in aromatic, acids, bases and resin content. They hypothesized that the oxidative degradation is probably preceded by autoxidation through a free radical mechanism. But they acknowledged that coal-derived liquids are so complex that it may be futile to attempt to define the actual mechanism.

In another study, Karn et al. [42] obtained similar results when they studied the aging characteristics of raw coal liquids produced from West Virginia coal by the SYNTHIOL PROCESS. Viscosity was the primary measure of degradation. The samples were aged at various temperature, storage time, atmosphere of oxygen, nitrogen, or air, stirring or no stirring, and light or darkness. They found a similar trend for all storage temperatures, that samples stored under oxygen atmosphere had a larger viscosity increase than samples stored in nitrogen or air. They also found that light had no effect on the viscosity increase of the sample. However, unstirred samples stored under oxygen or air had a smaller increase in viscosity than stirred samples in the same atmosphere.

Solvent analysis of fresh and stored samples aged under oxygen showed a decrease in pentane soluble and benzene soluble fractions with a corresponding increase in benzene insoluble fraction. There were no changes in component distribution in samples aged under nitrogen atmosphere.

Hara et al. [43] studied two different kinds of solvent refined coal (SRC) by aging SRC II middle distillate (boiling point range 450 - $565 \mathrm{~K}$ ) and 30/70 (wt.\%) blend of SRC I (solid product) and SRC II. Viscometric, ultimate analysis, solvent separation, gel permeation chromatography and NMR measurements were used 
to monitor the aging properties of the SRC liquids. They found that the viscosity of the blend increased significantly with oxygen bubbling. They proposed that oxidative coupling of phenols is the aging mechanism for both the blend and the middle distillate.

Kershaw and Gray [44] prepared coal liquids by laboratory hydropyrolysis of coal. They separated the toluene soluble product and further divided it into oil and asphaltenes. The samples were aged in test tubes in the dark at various temperatures with either oxygen or nitrogen atmosphere. Deterioration was measured by changes in viscosity and the increase in viscosity was again found to be greater on samples stored under an oxygen atmosphere than in nitrogen.

Brinkman [45] and Brinkman and Bowden [46] studied the storage stability of a variety of fuels and distillation cuts from petroleum, coal, oil shale and tar sands. The tests were conducted at $43{ }^{\circ} \mathrm{C}$ for periods of up to 32 weeks with aeration every four weeks. Formation of gum was measured after each storage period. Three naphthas from coal-derived samples had a large increase in gum content during the first 8 weeks of storage followed by a more gradual increase to 32 weeks. Elemental analyses of gum samples showed that there was a significant increase in oxygen content of the gum over the original fraction.

Wright and Weimer [47] performed stability studies of the chemical composition and biological activity of SRC I and SRC II liquids. The concentrations of major sample components were monitored as a function of storage time, temperature, dilution, atmosphere and light. Chemical composition was monitored using high resolution gas chromatography (GC) and gas chromatography/mass 
spectrometry (GC/MS). There were no changes in chemical composition and biological activity on samples stored at $4{ }^{\circ} \mathrm{C}$, inert containers, under nitrogen atmosphere, in the dark, and stored for 2 years (unaccelerated storage). Stored samples diluted with methylene chloride, with ambient light, under air atmosphere and room temperature degraded after one year of storage. The chemical constituents degraded were hydroxylated polycyclic aromatic hydrocarbons (PAH) and hydrogenated PAH. Samples stored at higher temperatures (accelerated storage) showed significant changes in chemical composition. Components which showed consistent degradation over time were hydroxylated $\mathrm{PAH}$ and partially hydrogenated $\mathrm{PAH}$.

\subsection{Basis of Instability}

It is evident from the literature that the instability of fuel is attributed to chemical reactions such as polymerization of unsaturated hydrocarbons. Some reactive organic compounds of sulfur and nitrogen, and oxygen in the fuel are thought to be responsible for fuel degradation. These reactions are accelerated by an increase in storage temperature.

The general measure of fuel instability is the amount of gum formation. The faster the rate of gum formation, the more unstable the fuel. Other measures of instability have been developed but are still correlated with gum formation. Viscosity, for example, depends on particulate or formation of gum. As the fuel ages, soluble gum formation increases and viscosity also increases. The color of the sample or light transmission techniques depends on gum formation. The larger the amount of gum, the darker the color of the fuel, which results in a decrease in 
light transmission with time. Oxygen absorption is a good measure of instability. It was found that the rate of gum formation is a function of the amount of oxygen present in the sample [9]. Light scattering techniques are based on the principle that as fuel ages, the particle's precursors to gum formation grow and scatter light.

\subsection{Filtrate Analysis over Gum Analysis}

Almost all fuel stability studies have been focused on gum analysis. While it is very interesting to know the gum composition, it will be very helpful to obtain information about the filtrate composition during storage. The disappearance of one compound class with time corresponding to the increase in gum formation would suggest that the particular compound class may be responsible for the deposition. This information may be an important tool in storage stability studies and fuel instability control.

In this study, the chemical analyses was focused on the filtrate analysis of coal liquid products before and after aging. Since instability is based on the chemical reactions among the fuel constituents, monitoring the changes in coal liquid composition with storage time will indicate the state of stability or quality of the sample. 


\subsection{EXPERIMENTAL METHODS}

The methods developed by Padlo, Kugler and Subramanian $[48,49]$ to characterize coal liquids quantitatively were used for the present stability analysis. Simulated distillation with an evaporative light-scattering detector (ELSD) provides a direct measure of high-boiling fractions of coal-derived liquids. The HPLChydrocarbon-compound-class analysis provides an automated, one-step procedure to separate and quantify heavy coal liquids into 6 compound classes. Figure 3.1 shows a picture of the HPLC system used in this study. The details on the equipment and procedures used are provided in the following sections.

\subsection{Simulated Distillation by ELSD}

The changes in boiling point distribution of coal liquids were determined using the simulated distillation by ELSD (Varex Mark II A). The experimental setup for the simulated distillation method is shown in Figure 3.2. The mobile phase is pumped to the autosampler (Varian 9095) using a Varian 9010 three solvent delivery system. After the sample is injected by the autosampler, it passes through a PEEK tubing (Supelco). This tubing provides a back pressure for the pump in order to maintain a constant flowrate of the mobile phase. The ELSD functions by transforming the HPLC solvent stream into a solvent spray in the nebulizer. The spray passes through a heated drift tube where the solvent and light products evaporate. Higher-boiling materials remain as small, micron-sized, discrete particles and are detected by light scattering $[48,49]$. Figure 3.3 shows the block diagram for the ELSD. 


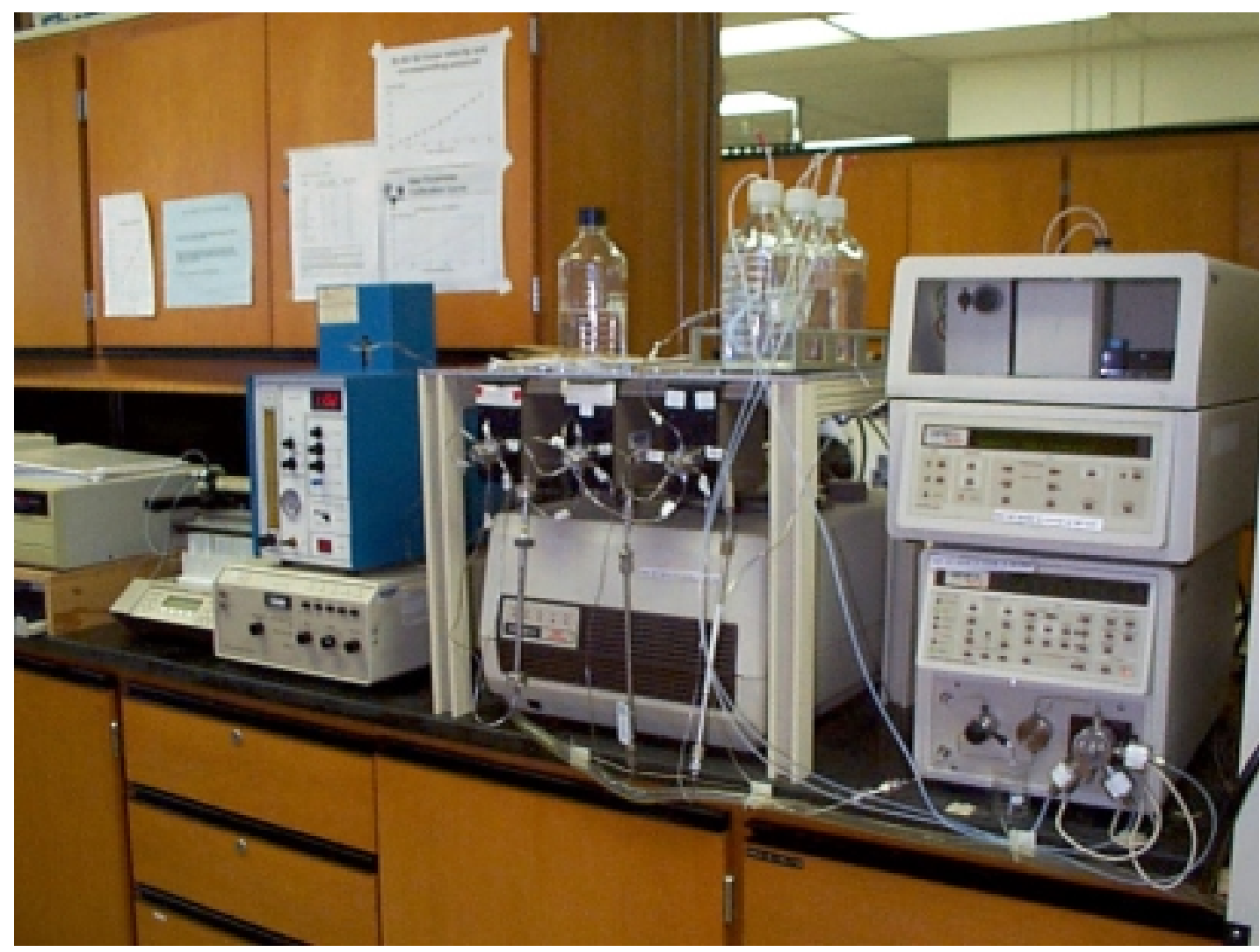

Figure 3.1 - High Performance Liquid Chromatography (HPLC) Used in the Stability Analysis of Coal Pyrolysis Liquids 


\section{Solvent \\ Reservoir}

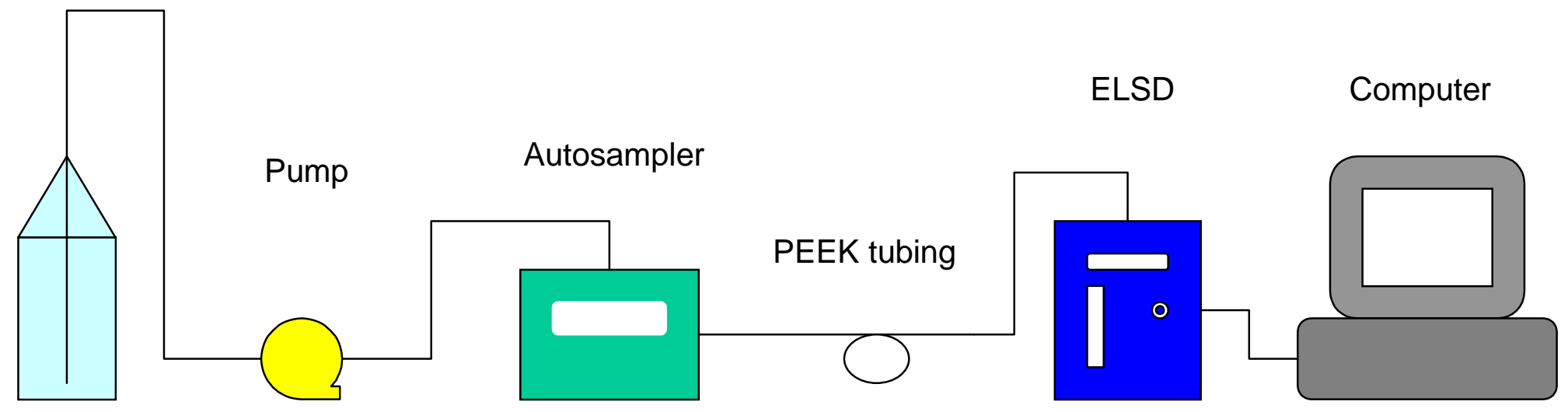

Figure 3.2 - The Boiling Point Distribution Setup 


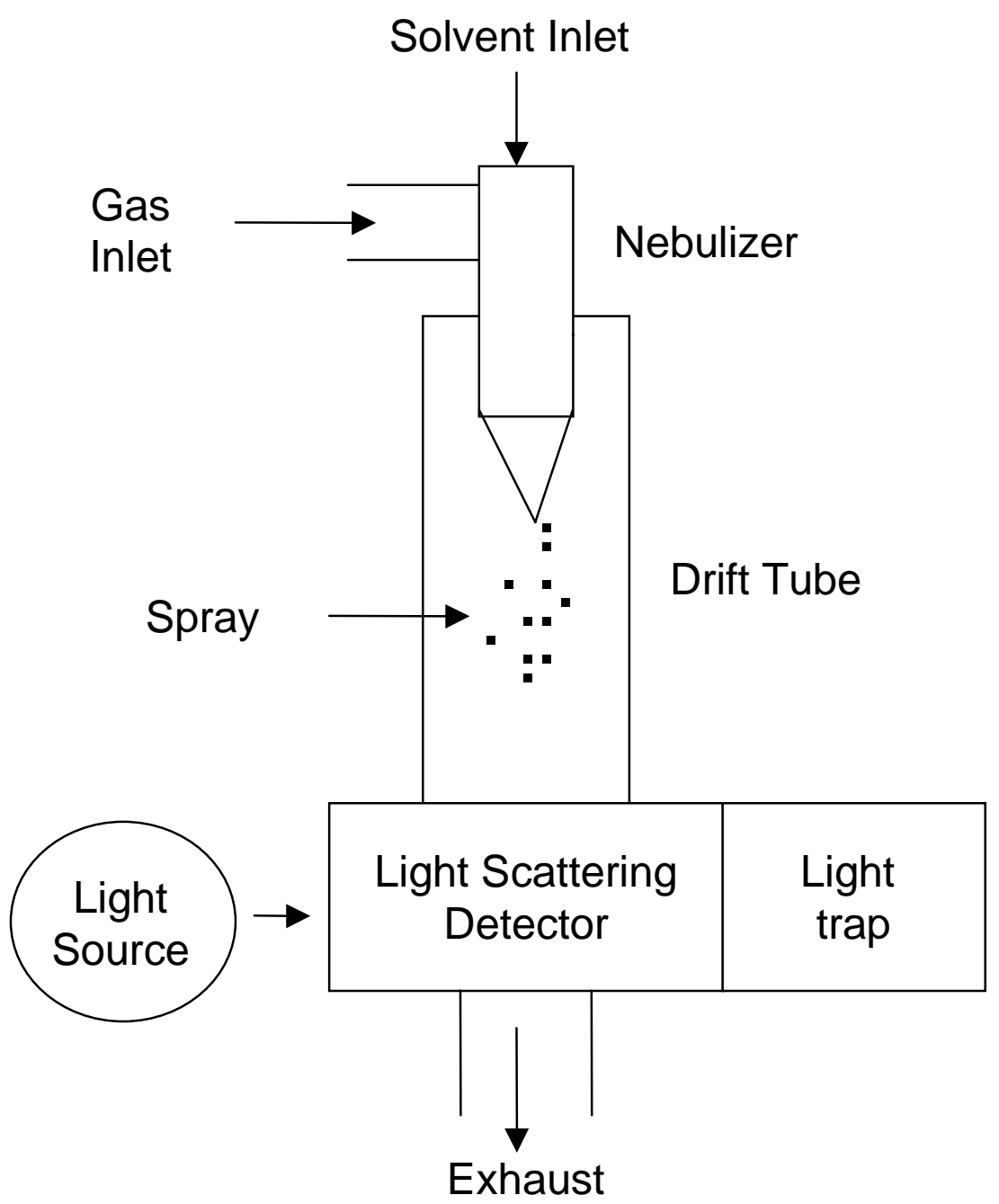

Figure 3.3 - Block Diagram of the Evaporative Light-Scattering Detector 
Padlo and Kugler [48] found that alkanes make excellent mass standards for the quantification of heavy oils using the ELSD. The alkane standards that were chosen gave a full response at a specified drift-tube temperature. When the detector is operated at $40{ }^{\circ} \mathrm{C}$, all constituents of the sample with a boiling point above $315^{\circ} \mathrm{C}$ will be detected. When operating at $80^{\circ} \mathrm{C}, 115^{\circ} \mathrm{C}$, and $150^{\circ} \mathrm{C}$, all of the constituents of the sample with a boiling point above $379^{\circ} \mathrm{C}, 435^{\circ} \mathrm{C}$, and 482 ${ }^{\circ} \mathrm{C}$, respectively, will be detected. For example, to determine the fraction of the sample having a boiling point greater than $315^{\circ} \mathrm{C}$, the drift tube temperature of the ELSD is set at $40^{\circ} \mathrm{C}$. If the sample was prepared at a concentration of $3.0 \mathrm{mg} / \mathrm{mL}$ and gave the same area counts as with the alkane standard at $2.0 \mathrm{mg} / \mathrm{mL}$, then $66.6 \%$ of the sample has a boiling point greater than $315^{\circ} \mathrm{C}$ and $33.3 \%$ of the sample has a boiling point less than $315^{\circ} \mathrm{C}$.

\subsection{HPLC Hydrocarbon Compound Class Analysis}

The HPLC hydrocarbon compound class analysis was used to monitor the changes in hydrocarbon compound class composition of the coal liquids. The method consists of a three-column high performance liquid chromatograph which separates hydrocarbon liquids into aliphatic, aromatic and polar fractions. The setup for this method is the same as the simulated distillation method shown in Figure 3.2 except that the PEEK tubing is replaced with three columns connected in series. One of the three columns used is the 2, 4-dinitroanilinopropyl silica (DNAP) column $(30 \times 0.46 \mathrm{~cm}$., ES Industries). This column separates the aromatics by ring size $[50,51]$. 
Most HPLC methods require removal of polar compounds prior to chromatography $[52,53]$. However, this three-column-HPLC technique does not need any pretreatment steps to remove polar compounds prior to HPLC analysis. The addition of a DIOL-silica column $(25 \times 0.46 \mathrm{~cm}$., Supelco $)$, which contains hydroxyl groups, placed in front of the DNAP column traps the polar compounds preventing them from being bound to the DNAP column.

The third column is the Partisil-amino-cyano (PAC) column $(25 \times 0.46 \mathrm{~cm}$., Whatman), which contains both amino and cyano functional groups. It separates saturates from the one-ring aromatics. The PAC column has stronger chargetransfer properties than the DNAP column [52].

Figure 3.4 shows the arrangement of the three columns in the operating system. Each column switching unit is composed of a 6-port valve, a column, and a bypass loop (a short segment of stainless steel tubing). The 6-port valves are controlled by relay switches found on a Varian 9010 solvent delivery system. Each valve is operated in one of the two positions: the on-line position which directs solvent flow through the column, and the bypass position which directs flow through the bypass loop.

During the first nine minutes, the solvent flows through all three columns in series (DIOL, DNAP, PAC). The polar molecules are adsorbed in the DIOL column; the multi-ring molecules are trapped in the DNAP column; and the one-ring aromatics and the saturates pass through the DIOL and DNAP columns and are separated at the PAC columns. 


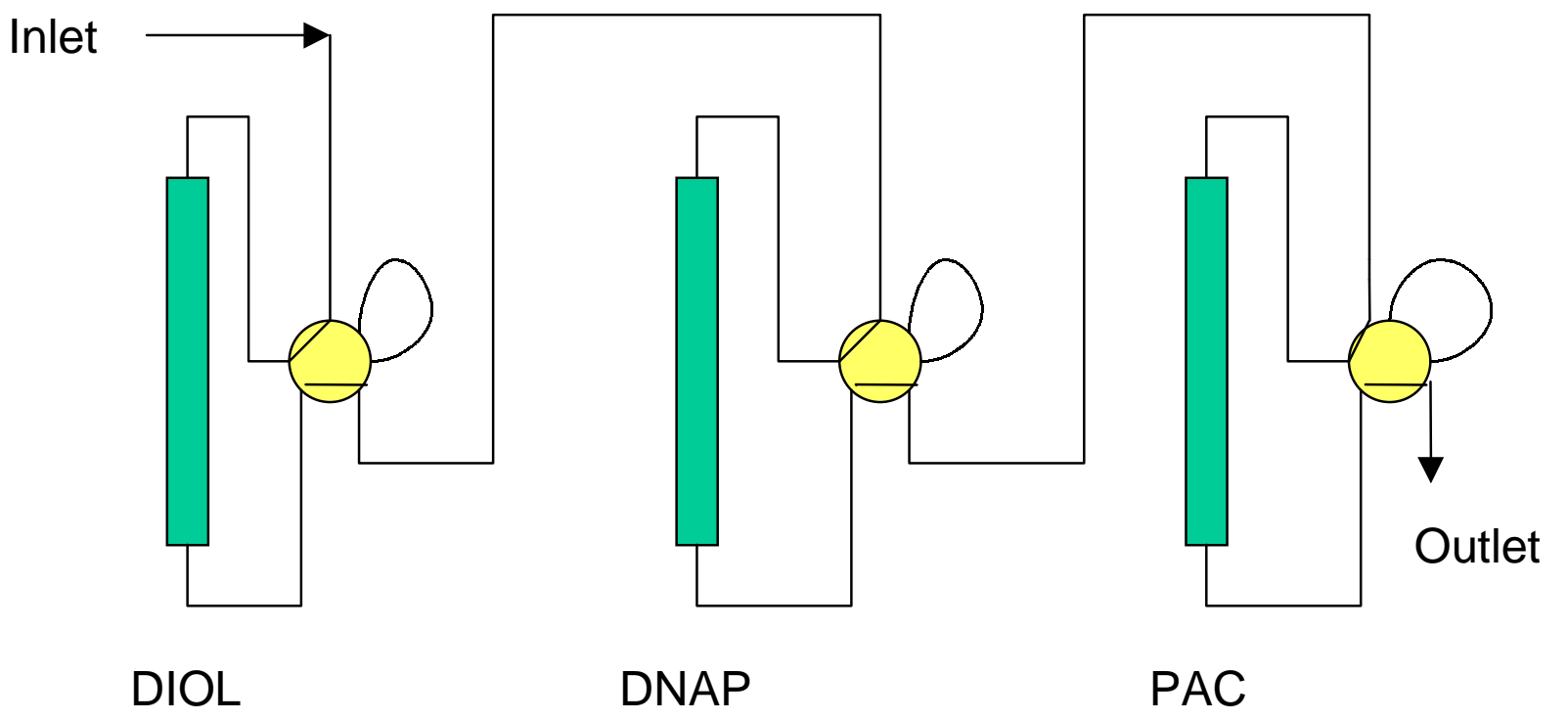

Figure 3.4 - The Arrangement of the Three Columns in the HPLC System 
The columns in series configuration are eluted one column at a time. The PAC column is eluted first with pentane solvent from 9 to 20.25 minutes giving the saturate fraction and the one-ring aromatic fraction. The DNAP column is eluted second using a pentane-to-methylene-chloride gradient from 20.26 to 56.52 minutes giving the two-, three- and four-ring aromatic fractions. The polar molecules are eluted from the DIOL column with a pentane-to-methylene-chloride / isopropanol gradient from 56.53 to 76.52 minutes. Finally, there is equilibration of all three columns with pentane from 76.53 to 85 minutes. Table 3.1 shows the timing and mobile phase composition for a pumping rate of $1.2 \mathrm{~mL} / \mathrm{min}$.

The results of Padlo, Subramanian, and Kugler [49] showed that the ELSD is a non-linear detector. The peak width has a significant influence on the area counts for a given compound. They have developed a power law model to linearize the response from the ELSD. This model was used to describe the mathematical relationship between sample concentration and detector signal for the ELSD. By using power law counts, instead of area counts obtained directly from the ELSD, the non-linear ELSD becomes a pseudo-linear detector. A computer program was created by Padlo and Kugler [48] which converts the response from the ELSD into power law counts. From the linear plot of power law counts vs concentration of alkane standards, one can determine the concentration of each of the different 6 compound classes contained in the oil sample. 
Table 3.1. Columns and Solvent Compositions Used for Hydrocarbon Compound Class Analysis. Solvent Flowrate is at $1.2 \mathrm{~mL} / \mathrm{min}$.

\begin{tabular}{|c|c|c|c|c|c|c|c|}
\hline \multirow[t]{2}{*}{ Time (min) } & \multirow[t]{2}{*}{ Columns on-line } & \multicolumn{3}{|c|}{ Initial Solvent Concentration } & \multicolumn{3}{|c|}{ Final Solvent Concentration } \\
\hline & & Pentane & $\mathrm{CH}_{2} \mathrm{Cl}_{2}$ & IPA & Pentane & $\mathrm{CH}_{2} \mathrm{Cl}_{2}$ & IPA \\
\hline 0 to 9.00 & DIOL, DNAP, PAC & 100 & 0 & 0 & 100 & 0 & 0 \\
\hline 9.00 to 14.00 & PAC & 100 & 0 & 0 & 100 & 0 & 0 \\
\hline 14.00 to 15.25 & PAC & 100 & 0 & 0 & 95 & 5 & 0 \\
\hline 15.25 to 20.25 & PAC & 95 & 5 & 0 & 95 & 5 & 0 \\
\hline 20.25 to 38.17 & DNAP & 95 & 5 & 0 & 40 & 60 & 0 \\
\hline 38.17 to 42.34 & DNAP & 40 & 60 & 0 & 0 & 100 & 0 \\
\hline 42.34 to 49.02 & DNAP & 0 & 100 & 0 & 0 & 100 & 0 \\
\hline 49.02 to 50.27 & DNAP & 0 & 100 & 0 & 100 & 0 & 0 \\
\hline 50.27 to 56.52 & DNAP & 100 & 0 & 0 & 100 & 0 & 0 \\
\hline 56.52 to 57.77 & DIOL & 100 & 0 & 0 & 0 & 95 & 5 \\
\hline 57.77 to 64.02 & DIOL & 0 & 95 & 5 & 0 & 95 & 5 \\
\hline 64.02 to 65.27 & DIOL & 0 & 95 & 5 & 0 & 100 & 0 \\
\hline 65.27 to 70.27 & DIOL & 0 & 100 & 0 & 0 & 100 & 0 \\
\hline 70.27 to 71.52 & DIOL & 0 & 100 & 0 & 100 & 0 & 0 \\
\hline 71.52 to 76.53 & $\mathrm{DIOL}$ & 100 & 0 & 0 & 100 & 0 & 0 \\
\hline 76.53 to 85.00 & DIOL, DNAP, PAC & 100 & 0 & 0 & 100 & 0 & 0 \\
\hline
\end{tabular}




\subsection{Coal Liquids}

The coal sample used in this study was Powellton Coal (WV GS 13421). Its proximate analysis by weight is as follows: $0.98 \%$ moisture, $67.87 \%$ fixed carbon, $27.96 \%$ volatile matter and $3.19 \%$ ash. The coal liquids used were obtained by hydropyrolysis of Powellton Coal using laboratory tubing bomb reactors. The reaction was carried out without a catalyst, with a hydrogen pressure of 1100 psig cold, and at a temperature of $450^{\circ} \mathrm{C}$ for one hour. The product was washed from the cooled reactor with hexane. Oil from the solid residue was extracted with boiling hexane in a Soxhlet extractor overnight. The hexane solutions were filtered and evaporated using a rotary evaporator (Buchler Instruments) to give a concentrated hexane-soluble product. The complete coal liquefaction procedure is described in Appendix 1.

\subsection{Aging of Coal Liquids}

The concentrated hexane-oil solution was filtered using a syringe filter with a $0.2 \mu \mathrm{m}$ pore PTFE membrane (Supelco). Several $10 \mathrm{~mL}$ hexane-oil samples were placed in preweighed borosilicate sample vials, sealed, and stored at 2, 21 and $32{ }^{\circ} \mathrm{C}$ under flourescent light. The samples were stored with approximately the same amount of air that is trapped in the bottle after it is sealed. The samples were not aerated during storage.

\subsection{Gum Measurements}

Stored samples were unsealed and filtered using a preweighed syringe filter. The sample vials were rinsed with hexane several times to dissolve all hexane- 
soluble oil left inside the vials. The rinse solutions were also filtered. Both the sample vial and the syringe filter were vacuum dried at room temperature until constant weight to give the weight of the hexane insolubles (gum) produced at that particular sampling time. One $10 \mathrm{~mL}$ volume taken from the bulk concentrated hexane-oil solution was vacuum dried at room temperature until constant weight to give the actual weight of the hexane-soluble oil in a $10 \mathrm{~mL}$ sample. The percentage of gum formed at a particular sampling time was obtained by determining the ratio of the weight of gum formed at that sampling time and the actual weight of oil in a $10 \mathrm{~mL}$ sample. In equation form,

$$
\% \text { weight gum }=\frac{G}{M} \times 100 \%
$$

where $\mathrm{G}=$ weight of gum formed in a $10 \mathrm{~mL}$ sample at a particular sampling time, $M=$ actual weight of oil in a $10 \mathrm{~mL}$ sample.

\subsection{Compound Class Measurements}

The ELSD responses for saturate, aromatic and polar compounds were converted into power law counts using the computer program developed by Padlo and Kugler [48]. From the standard curve, the concentration for each compound class in a $10 \mathrm{~mL}$ sample was obtained. The weight percentage of a particular compound class was determined by finding the ratio of the concentration of that compound class and the concentration of the oil in a $10 \mathrm{~mL}$ sample. In equation form, 
$\%$ weight of a compound class $=\frac{C}{D} \times 100 \%$

where $\mathrm{C}=$ the concentration of a particular compound class in a $10 \mathrm{~mL}$ sample, and $\mathrm{D}=$ the concentration of oil in a $10 \mathrm{~mL}$ sample.

\subsection{Hydrocarbon Class Identification}

Compound classes were identified using both retention data from standards and UV spectra measured on the Polychrome 9065 diode array detector. The detector has the ability to identify compound classes by measuring the UV spectrum of molecules eluting from the columns. 


\subsection{RESULTS AND DISCUSSION}

At the beginning of this study, a method for determining the molecular weight distribution of coal and petroleum-derived heavy oils was developed with a joint effort of Jisheng Zhu. The details of the Molecular Weight Distribution method are discussed in Section 4.1. Sections $4.2-4.9$ are the results obtained from the stability studies of coal liquids.

\subsection{Molecular Weight Distribution Method}

\subsubsection{Introduction}

In the analysis of coal and petroleum-derived heavy oils, the molecular weight distribution (MWD) is one of the important factors in judging their suitability as feedstocks for further processing into other products like transport fuels or raw materials for the chemical industry. Unfortunately, vapor-pressure osmometry provides the average molecular weight only. The most commonly used method for MWD is high performance size-exclusion chromatography (SEC) also known as gel permeation chromatography. In this method, the molecules of different sizes are separated according to their degree of penetration into the pores of gel packed into a column and the eluted materials are detected by using a UV or refractive index (RI) detector [56].

However, reliable MWD can only be obtained when the detector provides a similar response for each separated compounds or class of compounds [57]. None of the conventional HPLC detectors (UV, RI) meet this requirement [57]. It has been shown that there is up to $50 \%$ difference in the average $\mathrm{MW}$ observed with the 
UV detector for coal extract fractions [58]. This is true because coal molecules tend to absorb more strongly at longer wavelengths as MW increases [59]. The RI detector has a poor linearity for higher MW materials [59]. These problems led to studies of the applicability of ELSD in SEC of coal derivatives, heavy oils and polystyrenes [59, 60,61].

Bartle et al. [59] found that for MW above 300, the mass response of ELSD is almost uniform. They found that a variety of compounds like alkanes, polystyrenes and polyacenaphthylenes gave full response within $10 \%$ difference. They concluded that their ELSD was a linear mass detector for SEC and has considerable advantages over UV and RI detectors.

Although SEC is easy to use, this technique was designed for separating high-molecular-weight components $(\mathrm{MW}>2000)$ and non-ionic compounds. Moreover, SEC can be used effectively to separate simple mixtures when the components of the mixture have sufficient difference in molecular size [62]. Also, column problems like irreversible absorption are always present. These drawbacks prompted the development of a new method to measure the MWD of coal and petroleum-derived heavy oils using ELSD directly.

\subsubsection{Determination of the MW Detection Limits}

Coal or petroleum-derived heavy oils are composed of different types of compounds that have different solubilities. The responses of the different standard compounds and samples prepared in different solvents need to be considered. Figure 4.1 shows that different types of compounds give similar responses. This coincides with Bartle's [59] result. This is very important for the quantification of 


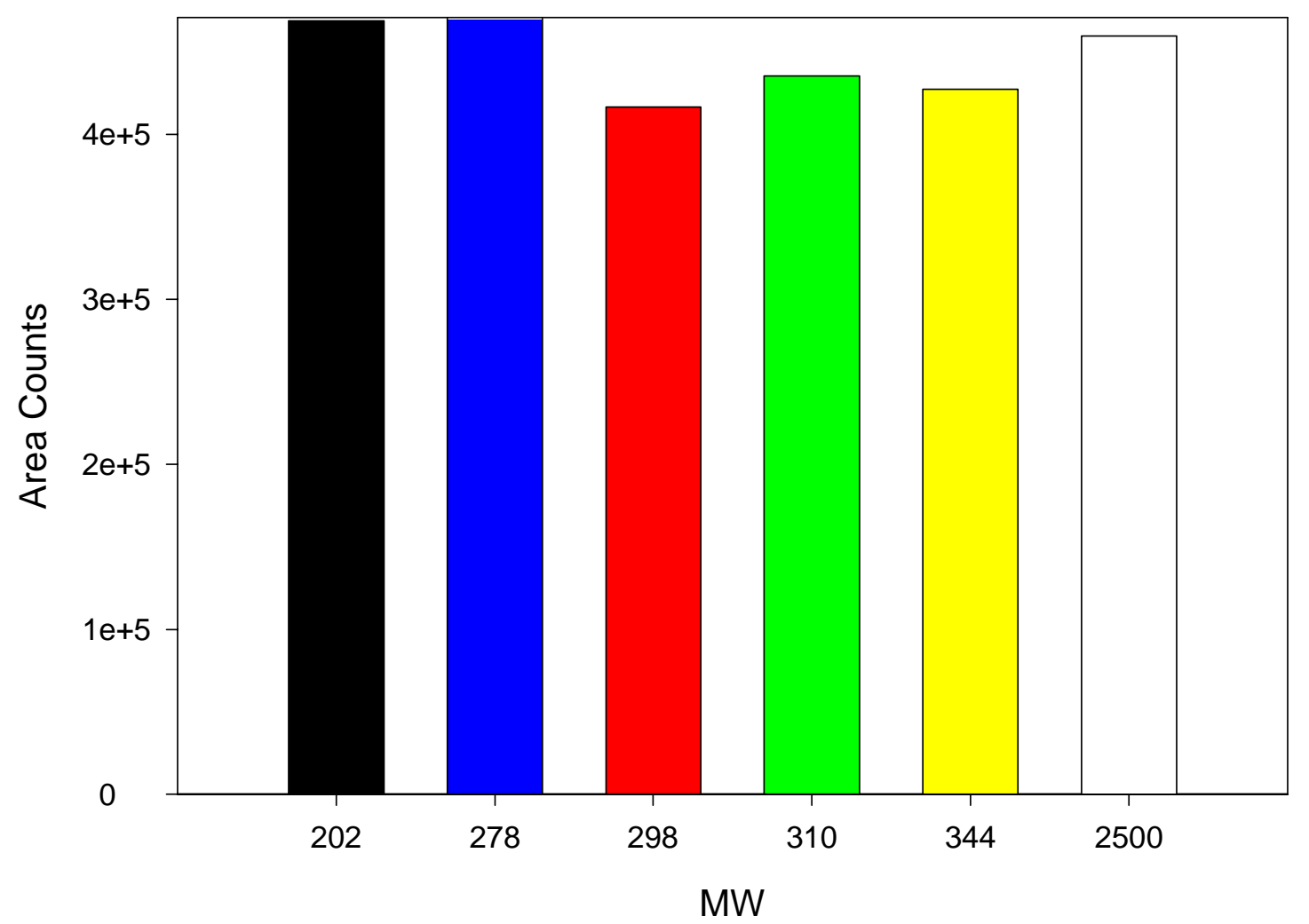

Figure 4.1 - Responses of Different Types of Compounds with Different MW 
samples because only one alkane standard was used for the calibration at each drift tube temperature setting.

Figure 4.2 shows the similar response of $n-C_{22}$ prepared using different solvents. This offers a wider range of selection of solvents for the preparation of samples.

Figure 4.3 shows the ELSD response vs. MW of an alkane series and Table 4.1 shows the alkane series used in this experiment. At $40{ }^{\circ} \mathrm{C}$, the lower MW alkanes up to $n-C_{16}(M W=226)$ showed zero response while the higher $M W$ alkanes beginning at $\mathrm{n}-\mathrm{C}_{20}(\mathrm{MW}=282)$ showed full response. $A$ transition region between $n-C_{16}$ and $n-C_{20}$ goes upward from zero response to full response. A similar trend is observed at different drift tube temperature $\left(80^{\circ} \mathrm{C}, 115^{\circ} \mathrm{C}, 150^{\circ} \mathrm{C}\right)$ settings. However, the sensitivity changes as a function of detector temperature. The detector gives a full response beginning at $n-C_{26}(M W=366)$ at $80^{\circ} \mathrm{C}, n-C_{32}$ $(\mathrm{MW}=450)$ at $115^{\circ} \mathrm{C}$, and $\mathrm{n}-\mathrm{C}_{36}(\mathrm{MW}=506)$ at $150^{\circ} \mathrm{C}$.

Figure 4.4 shows the ELSD response vs. MW for a series of aromatics and Table 4.2 shows the aromatic series used in this experiment. At $40^{\circ} \mathrm{C}$, the lower MW aromatics up to acenaphthene $(M W=154)$ showed zero response while the higher MW aromatics beginning at fluoranthene or pyrene $(M W=202)$ showed full response. A transition region between acenaphthene and fluoranthene or pyrene goes upwards from zero response to full response. A similar trend is observed at different drift tube temperature $\left(80^{\circ} \mathrm{C}, 115^{\circ} \mathrm{C}, 150{ }^{\circ} \mathrm{C}\right)$ settings. However, the sensitivity changes as a function of the detector temperature. The detector gives 


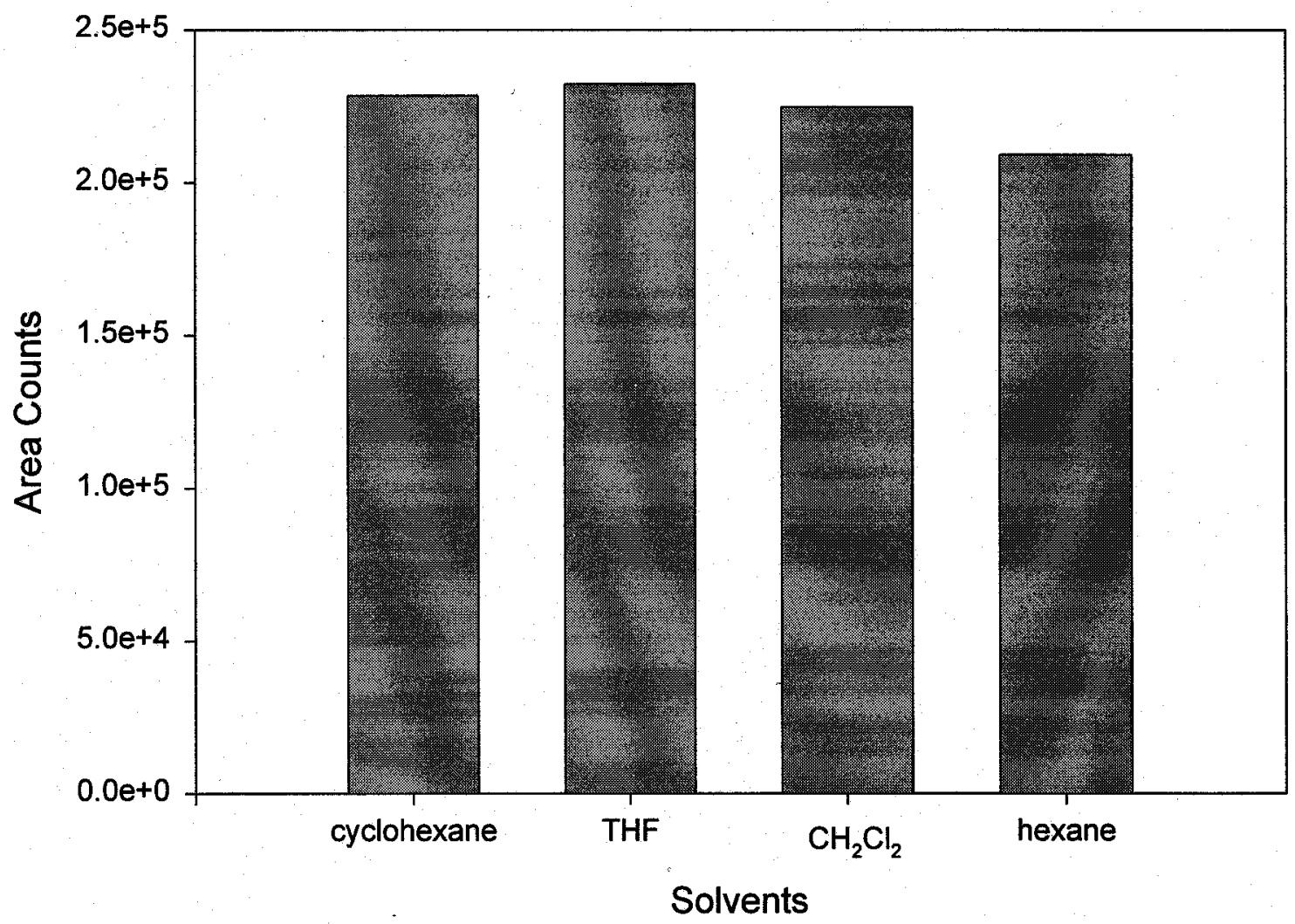

Figure 4.2 - Reponses of $\mathrm{C}_{22}$ in Different Solvents 


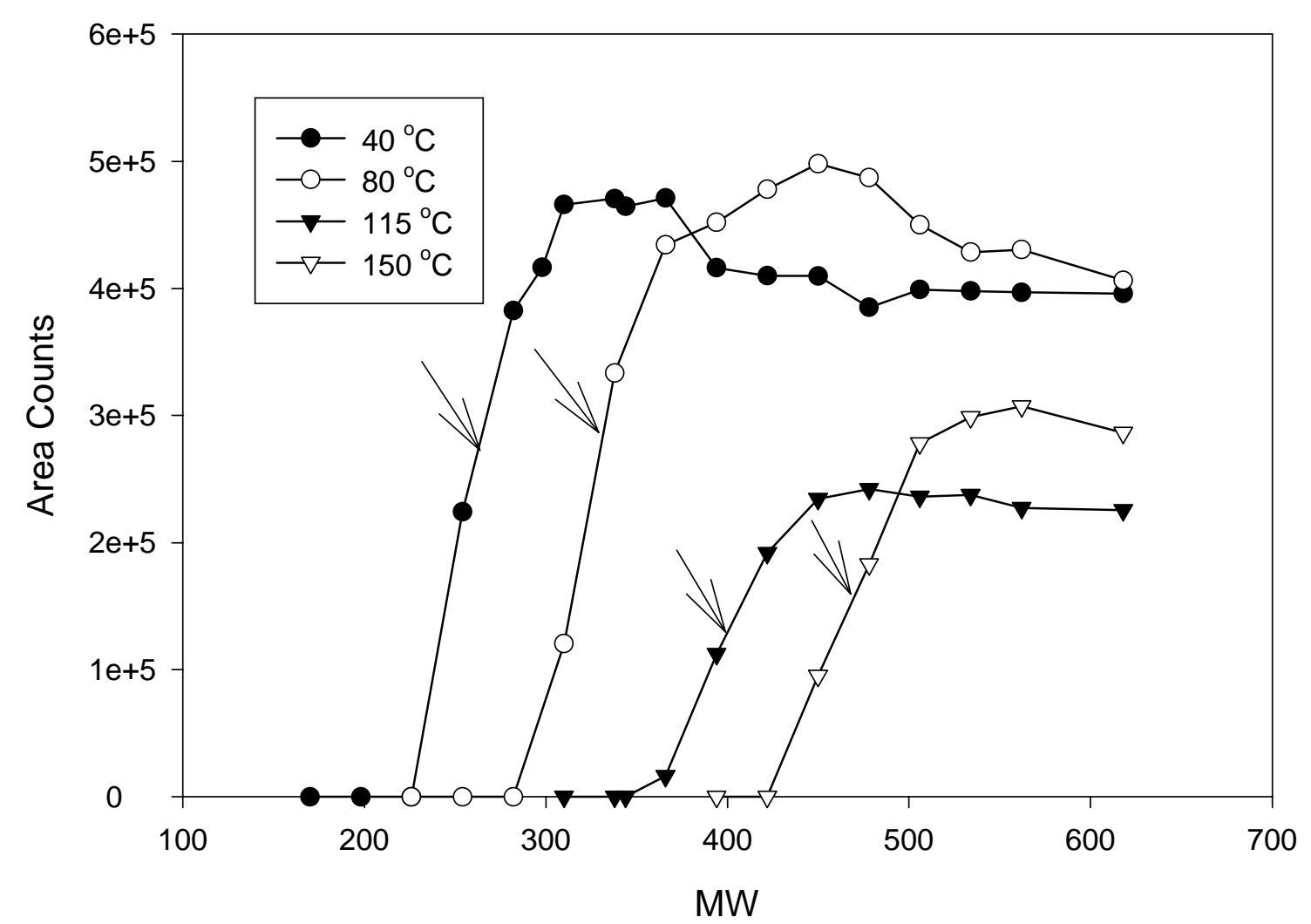

Figure 4.3 - Responses of a Series of n-Alkanes as a Function of MW (Arrows Represent the MW Detection Limits) 
Table 4.1. The Alkane Compounds Used in Obtaining the Data in Figure 4.3.

\begin{tabular}{|c|c|}
\hline Compound & MW \\
\hline $\mathrm{C}_{12}$ & 170 \\
\hline $\mathrm{C}_{14}$ & 198 \\
\hline $\mathrm{C}_{16}$ & 226 \\
\hline $\mathrm{C}_{18}$ & 254 \\
\hline $\mathrm{C}_{20}$ & 282 \\
\hline $\mathrm{C}_{22}$ & 310 \\
\hline $\mathrm{C}_{24}$ & 338 \\
\hline $\mathrm{C}_{26}$ & 366 \\
\hline $\mathrm{C}_{28}$ & 394 \\
\hline $\mathrm{C}_{30}$ & 422 \\
\hline $\mathrm{C}_{32}$ & 450 \\
\hline $\mathrm{C}_{34}$ & 478 \\
\hline $\mathrm{C}_{36}$ & 506 \\
\hline $\mathrm{C}_{38}$ & 534 \\
\hline $\mathrm{C}_{40}$ & 562 \\
\hline $\mathrm{C}_{44}$ & 618 \\
\hline & \\
\hline & \\
\hline
\end{tabular}




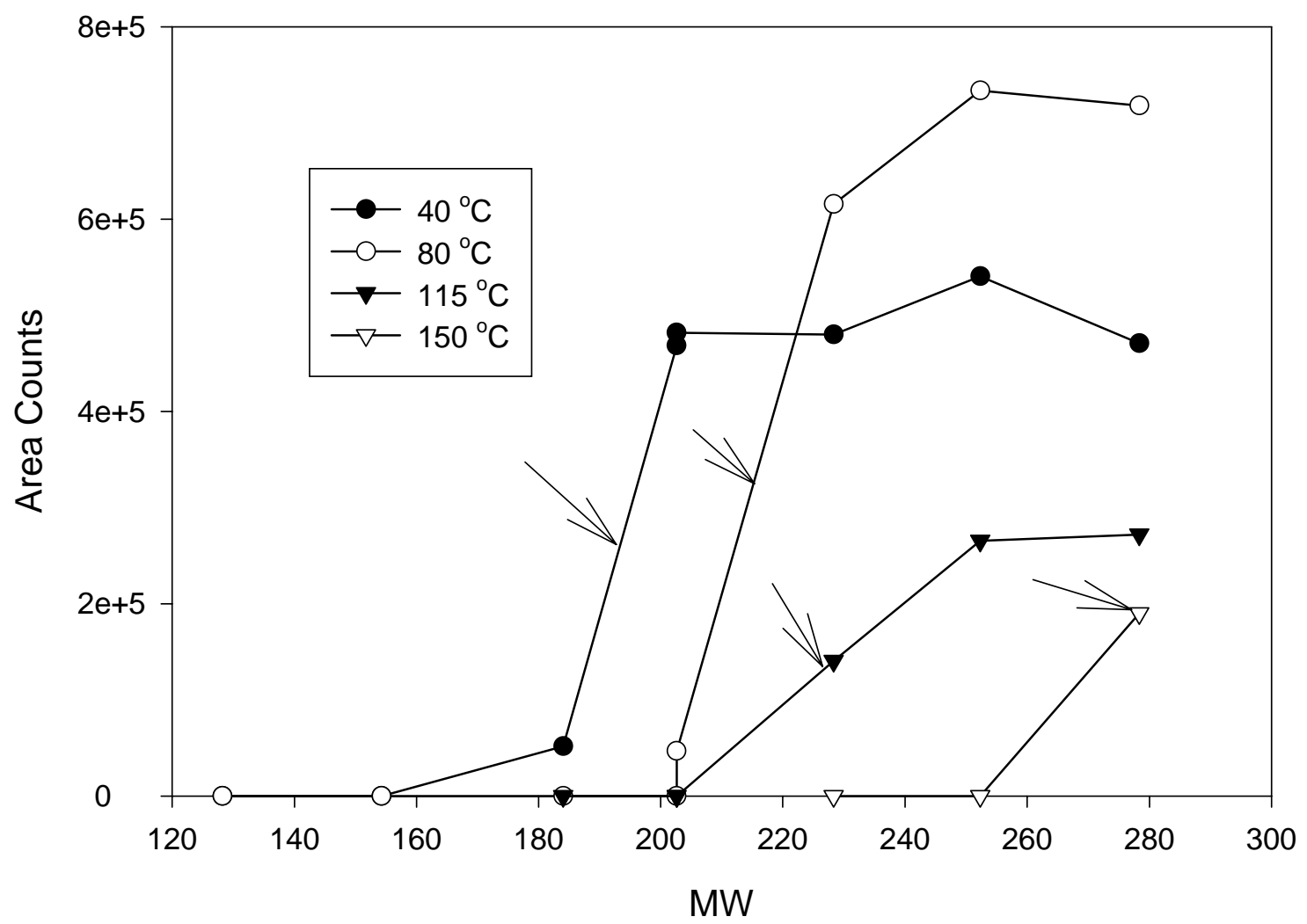

Figure 4.4 - Responses of a Series of Aromatics as a Function of MW (Arrows Represent the MW Detection Limits) 
Table 4.2. The Aromatic Compounds Used in Obtaining the Data in Figure 4.4.

\begin{tabular}{|c|c|}
\hline Compound & MW \\
\hline Naphthalene & 128 \\
\hline Acenaphthene & 154 \\
\hline Phenanthrene & 178 \\
\hline Anthracene & 178 \\
\hline Dibenzothiophene & 184 \\
\hline Pyrene & 202 \\
\hline Fluoranthene & 202 \\
\hline Chrysene & 228 \\
\hline Benzo(a)pyrene & 252 \\
\hline Dibenzo(a,h)anthracene & 278 \\
\hline
\end{tabular}

Table 4.3. The MW Detection Limits for the Alkane and Aromatic Series.

\begin{tabular}{|c|c|c|c|}
\hline $\begin{array}{c}\text { Temperature } \\
\left({ }^{\circ} \mathbf{C}\right)\end{array}$ & Alkane Series & Aromatic Series & Average \\
\hline 40 & 254 & 193 & 224 \\
\hline 80 & 324 & 215 & 270 \\
\hline 115 & 394 & 226 & 310 \\
\hline 150 & 464 & 278 & 371 \\
\hline
\end{tabular}


a full response beginning with chrysene $(\mathrm{MW}=228)$ at $80^{\circ} \mathrm{C}$, benzo(a)pyrene $(\mathrm{MW}$ $=252)$ at $115^{\circ} \mathrm{C}$, and dibenzo $(\mathrm{a}, \mathrm{h})$ anthracene $(\mathrm{MW}=278)$ at $150^{\circ} \mathrm{C}$.

The MW detection limit is defined here as the MW that is halfway between the transition region as indicated by the arrows in Figures 4.3 and 4.4. For example, for the alkane series at $40{ }^{\circ} \mathrm{C}$, the $\mathrm{MW}$ that is halfway through the transition region is 254 (the average of 226 and 282). Also, for the aromatics at 40 ${ }^{\circ} \mathrm{C}$, the MW that is halfway through the transition region is 193 (the average of 184 and 202). Similarly, the MW detection limits for the alkane series and the aromatic series at different drift tube temperature settings were determined based on this definition. However, at $150{ }^{\circ} \mathrm{C}$ for the aromatic series, 278 (dibenzo(a, h)anthracene) was selected as the MW detection limit because there were no soluble aromatic standards with MW greater than 278 available to run the experiment.

Since the method is aimed at determining the MWD of samples that are mixtures of alkanes and aromatics, the average MW detection limits were taken between the alkane and aromatic series. Table 4.3 shows the MW detection limits for the alkane and the aromatic series and their averages.

\subsubsection{Sample Calculations}

Figure 4.5 shows the calibration curve of $\mathrm{C}_{22}$ obtained at $40{ }^{\circ} \mathrm{C}$. The standard $\mathrm{C}_{22}$ was chosen because it gave a full response when the drift tube is set at $40{ }^{\circ} \mathrm{C}$. Assuming that the average MW detection limit of 224 at $40^{\circ} \mathrm{C}$ is correct, if $1.6 \mathrm{mg} / \mathrm{mL}$ sample mixture is injected at $40^{\circ} \mathrm{C}$ and gave an area count of 494,561 , then from the calibration curve (Figure 4.5), the sample has approximately 1.558 


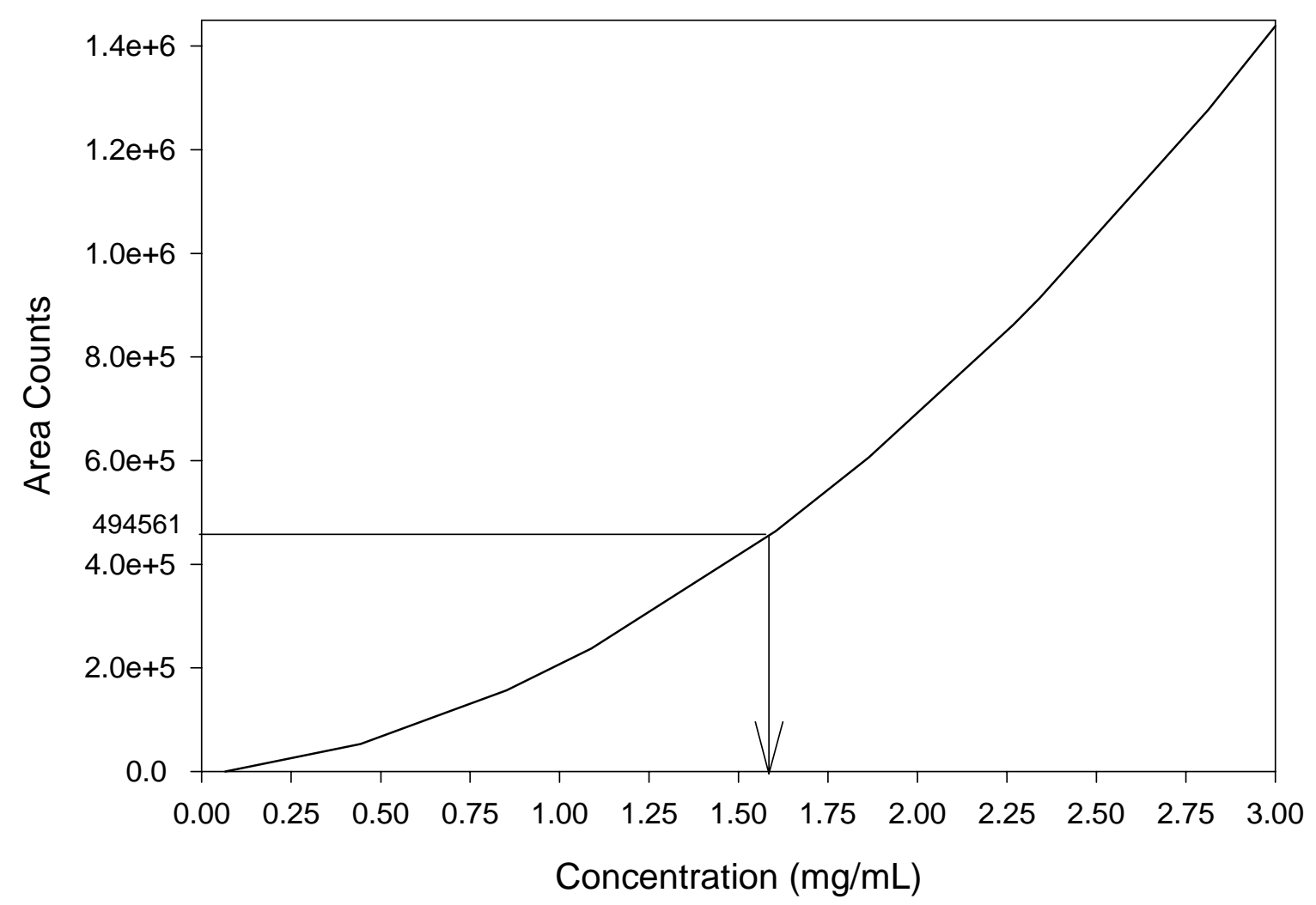

Figure 4.5 - Calibration Curve of $\mathrm{C}_{22}$ at $40{ }^{\circ} \mathrm{C}$ 
$\mathrm{mg} / \mathrm{mL}$ with MW greater than 224. Thus, $97 \%(1.558 / 1.6)$ of the sample injected has MW greater than 224 .

\subsubsection{Results from MWD Test}

Standard mixtures of alkanes and aromatics were tested to determine if the MWD method will work. Table 4.4 shows the results of the test. The first standard mixture of $0.8 \mathrm{mg} / \mathrm{mL} \mathrm{n}-\mathrm{C}_{16}(226)$ and $0.8 \mathrm{mg} / \mathrm{mL}$ acenaphthene (154) gave no response. This is true because the actual MW detection limit for alkanes at $40{ }^{\circ} \mathrm{C}$ is 254 which is greater than the MW of $\mathrm{n}-\mathrm{C}_{16}$ and the actual MW detection limit for aromatics at $40{ }^{\circ} \mathrm{C}$ is 193 which is also greater than the MW of acenaphthene. However, this is not the case if the average MW detection limit of 224 is adopted. Since half of the sample mixture contains $n-C_{16}$ which has a MW greater than 224, it should report that $50 \%$ has MW greater than 224 . Thus, the hypothesized average MW detection limit is incorrect.

The second standard mixture of $n-C_{20}(282)$ and naphthalene (128) suggests that approximately $45.1 \%(0.722 / 1.60)$ of the sample mixture has $\mathrm{MW}$ greater than 224. This time, the average MW detection limit holds true because only half of the mixture has MW greater than 224 which is $\mathrm{n}-\mathrm{C}_{20}$. Comparing the results with the actual detection limits, $\mathrm{n}-\mathrm{C}_{20}$ has a higher MW than the 254 (alkane detection limit) and naphthalene has a lower MW than 193 (aromatic detection limit). Thus, it is correct that only half of the mixture will be detected by the method.

The last standard mixture of $n-C_{22}(310)$ and pyrene (202) suggests that approximately $99.4 \%(1.59 / 1.60)$ of the sample mixture has MW greater than 224 . This is not correct because half of the mixture has MW less than 224 which is 
Table 4.4. Results from Testing the Standard Mixtures at $40^{\circ} \mathrm{C}$.

\begin{tabular}{|c|c|c|c|c|c|}
\hline Standard Mixture & $\begin{array}{l}\text { Average MW } \\
\text { Detection } \\
\text { Limit at } 40^{\circ} \mathrm{C}\end{array}$ & $\begin{array}{l}\text { Area } \\
\text { Counts } \\
\text { from } \\
\text { ELSD }\end{array}$ & $\begin{array}{c}\text { Concentration } \\
(\mathrm{mg} / \mathrm{mL}) \text { obtained } \\
\text { from Calibration } \\
\text { Curve }\end{array}$ & $\begin{array}{c}\text { Total } \\
\text { Concentration of } \\
\text { Standard Mixture } \\
(\mathrm{mg} / \mathrm{mL})\end{array}$ & $\begin{array}{l}\text { Percent } \\
\text { Weight } \\
\text { Detected by } \\
\text { ELSD }\end{array}$ \\
\hline $\begin{array}{l}0.8 \mathrm{mg} / \mathrm{mL} \mathrm{n}-\mathrm{C}_{16}(226)+ \\
0.8 \mathrm{mg} / \mathrm{mL} \text { acenaphthene } \\
(154)\end{array}$ & 224 & 0 & 0 & 1.6 & $0.0 \%$ \\
\hline $\begin{array}{l}0.8 \mathrm{mg} / \mathrm{mL} \mathrm{n}-\mathrm{C}_{20}(282)+ \\
0.8 \mathrm{mg} / \mathrm{mL} \text { naphthalene }(128)\end{array}$ & 224 & 106332 & 0.722 & 1.6 & $45.1 \%$ \\
\hline $\begin{array}{l}0.8 \mathrm{mg} / \mathrm{mL} \mathrm{n}-\mathrm{C}_{22}(310)+ \\
0.8 \mathrm{mg} / \mathrm{mL} \text { pyrene }(202)\end{array}$ & 224 & 515628 & 1.590 & 1.6 & $99.4 \%$ \\
\hline
\end{tabular}


pyrene. Therefore, the hypothesized average MW detection limit is incorrect. However, it should be noted that all the constituents of the sample will be detected because both $n-C_{22}$ and pyrene have $M W$ above the actual detection limits for alkanes (254) and aromatics (193). This is the reason for the $99.4 \%$ detection.

\subsubsection{Conclusions for the MWD Method}

This method can be used for samples which consist mainly of saturates like petroleum or wax. In this case, the alkane MW detection limits will be used to measure its MWD. This method can also be used for samples which consist mainly of polyaromatic hydrocarbons like coal-tar pitch or polycyclic aromatic compounds (PAC's). In this case, the aromatic MW detection limits will be used to measure its MWD. The advantages of using this method are: 1) the sample size for the determination is small, and, 2) the separation and adsorption problems can be avoided since no columns are used in the analysis. Since the detection limits in Figure 4.6 appears to be linear, this will allow the determination of other MW detection limit that is greater than or less than the detection limits already investigated.

Originally, this method was developed to analyze hydropyrolysis coal liquids. However, it was later found that the coal liquids used contain primarily aromatic and polar compounds. Handling samples that contain mixtures of aromatic and polar compounds are beyond the applicability of this method. The method is limited to samples which contain primarily saturates or aromatics. Thus, this method did not work out for samples of current interest. 


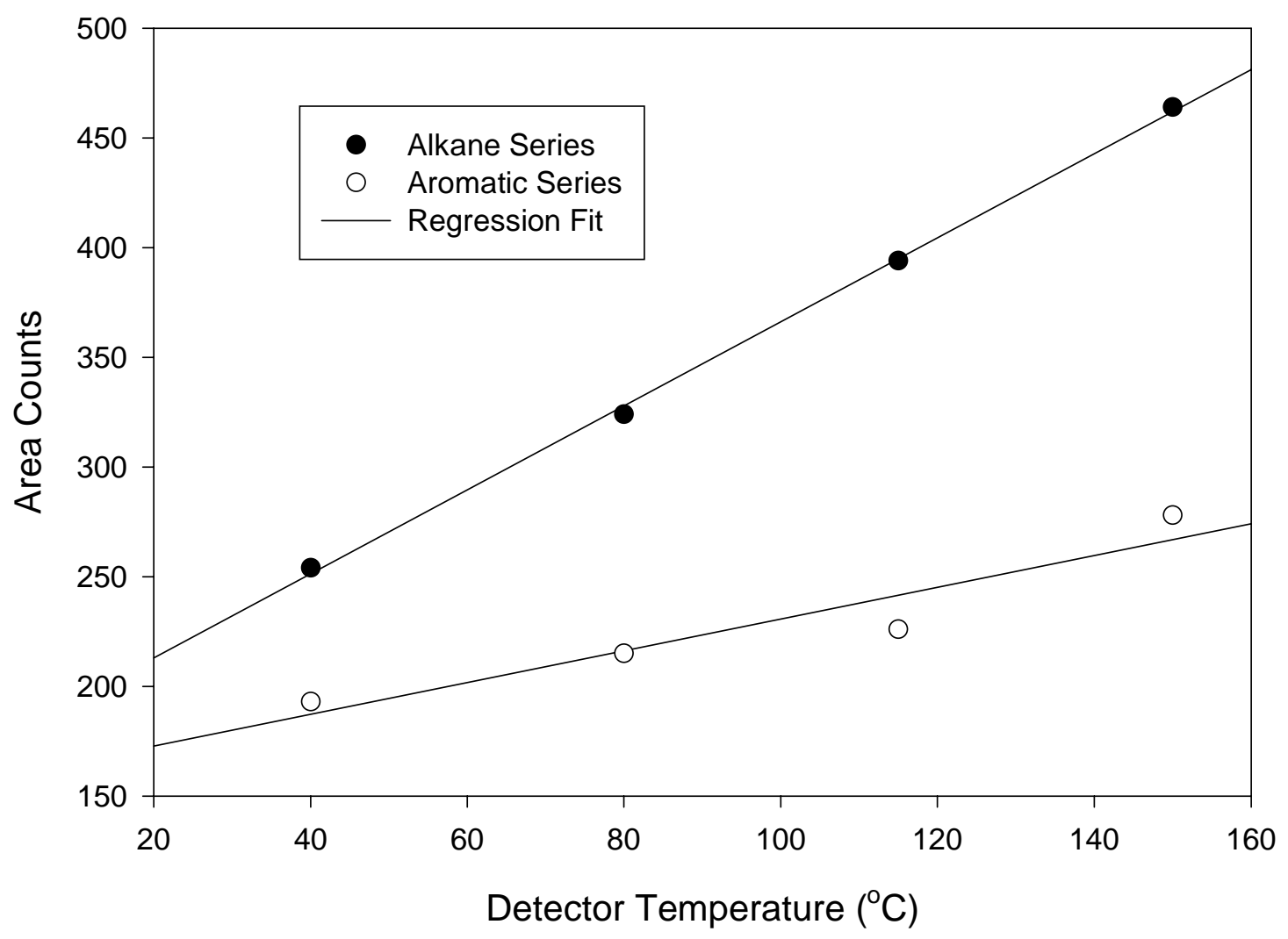

Figure 4.6 - MW Detection Limit as a Function of Detector Temperature 


\subsection{Coal Liquids}

The coal liquids were obtained by hydropyrolysis of Powellton Coal using laboratory tubing reactors as described in Section 3.3. Only the hexane-soluble product was used in this study. On the average, the total conversion obtained was $20 \%$. A sample calculation for the percent total conversion is shown in Appendix 2.

\subsection{Gum Formation}

\subsubsection{Effects of Storage Temperature}

When coal liquids are stored, gum formation occurs and this can be easily observed by visual examination. All coal liquid samples stored at $2{ }^{\circ} \mathrm{C}, 21^{\circ} \mathrm{C}$ and $32{ }^{\circ} \mathrm{C}$ became darker in color with time. Hexane insoluble materials began to deposit on the inner wall and at the bottom of the sample vials as early as three hours into storage. Figure 4.7 shows the picture of a fresh coal liquid sample and an aged (stored at $21^{\circ} \mathrm{C}$ for 72 hours) coal liquid sample. This result is consistent with literature that stored fuel undergoes color changes as a sign of degradation [1, $9,10,66]$

The gum deposited on the walls of the sample vials became thicker in appearance as time progressed. Figure 4.8 shows three sample vials that were washed several times with hexane after storage. They were taken at sampling times 3,24 , and 48 hours. These particular sampling times were chosen because the formation of hexane insoluble gum is greatest at the early stages of storage The changes are more pronounced at higher storage temperatures. Figures 4.8, 4.9 and 4.10 show the gum formed at different storage temperatures. 


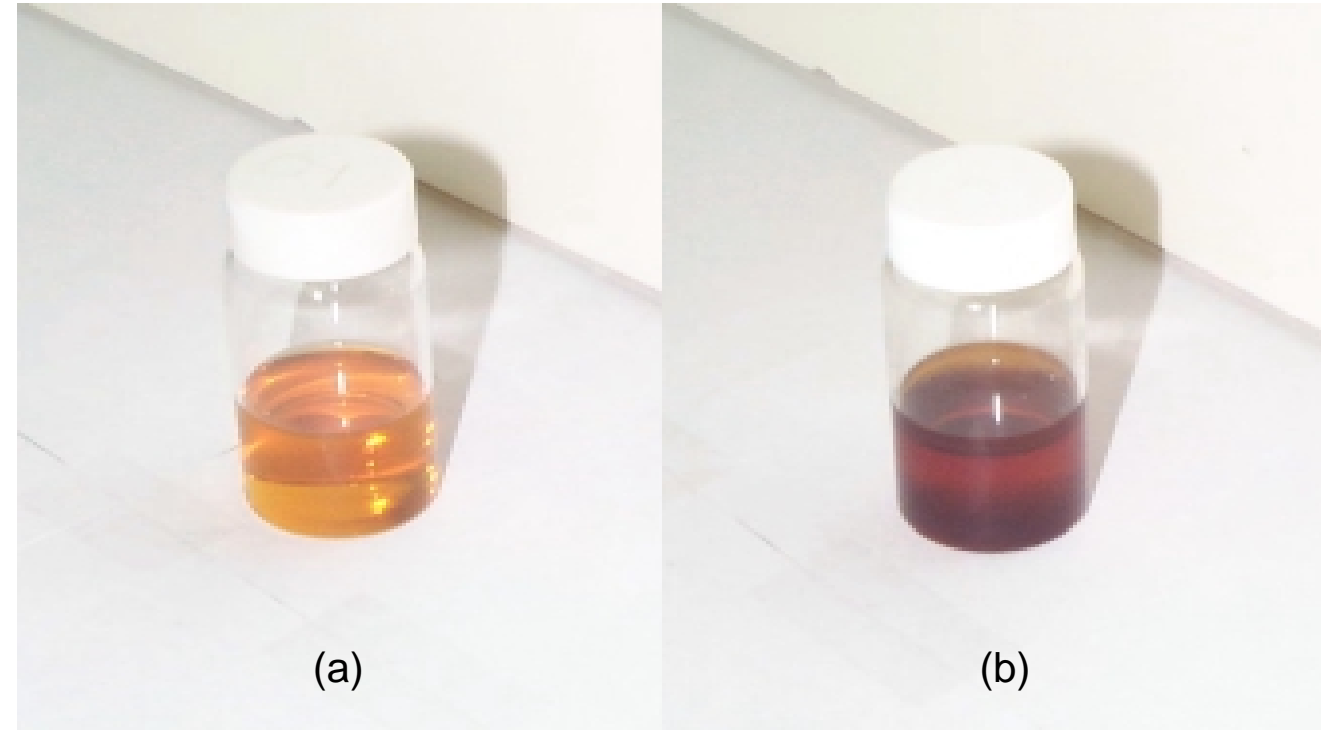

Figure 4.7 - (a) Picture of Fresh Coal Liquid Sample;

(b) Picture of Aged Coal Liquid Sample

Stored at $21^{\circ} \mathrm{C}$ for 72 hours 


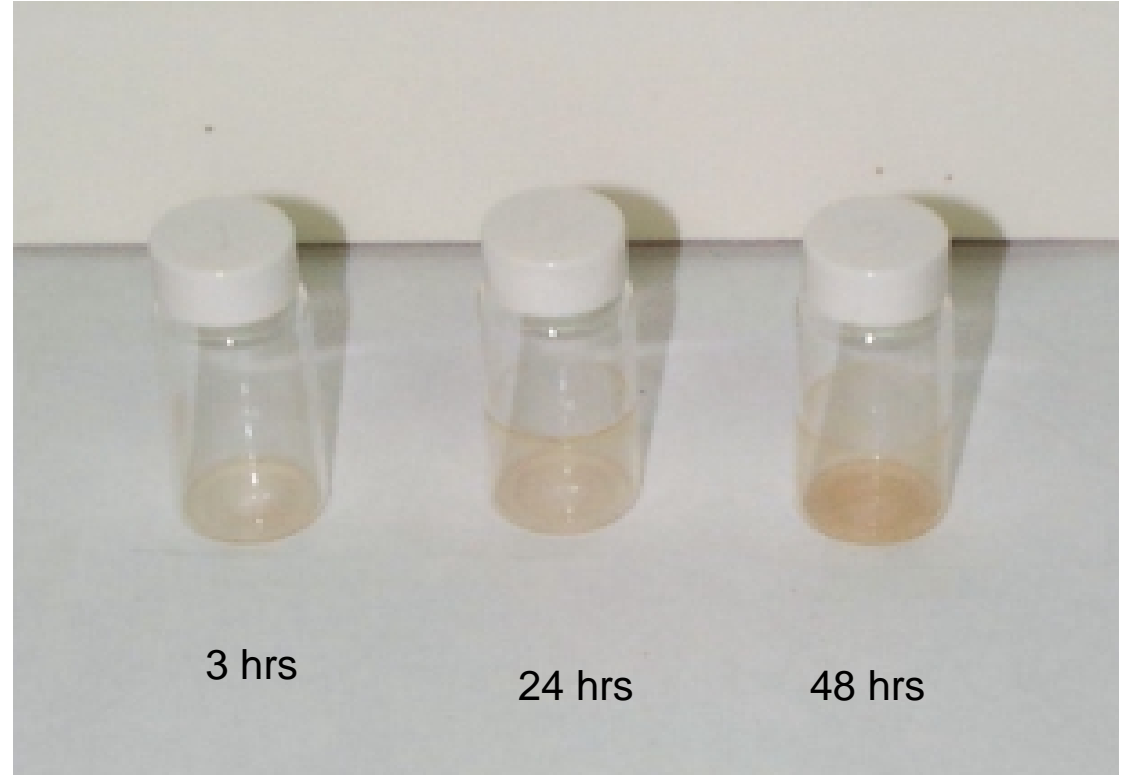

Figure 4.8 - Gum Formation in Samples Stored at $2{ }^{\circ} \mathrm{C}$ 


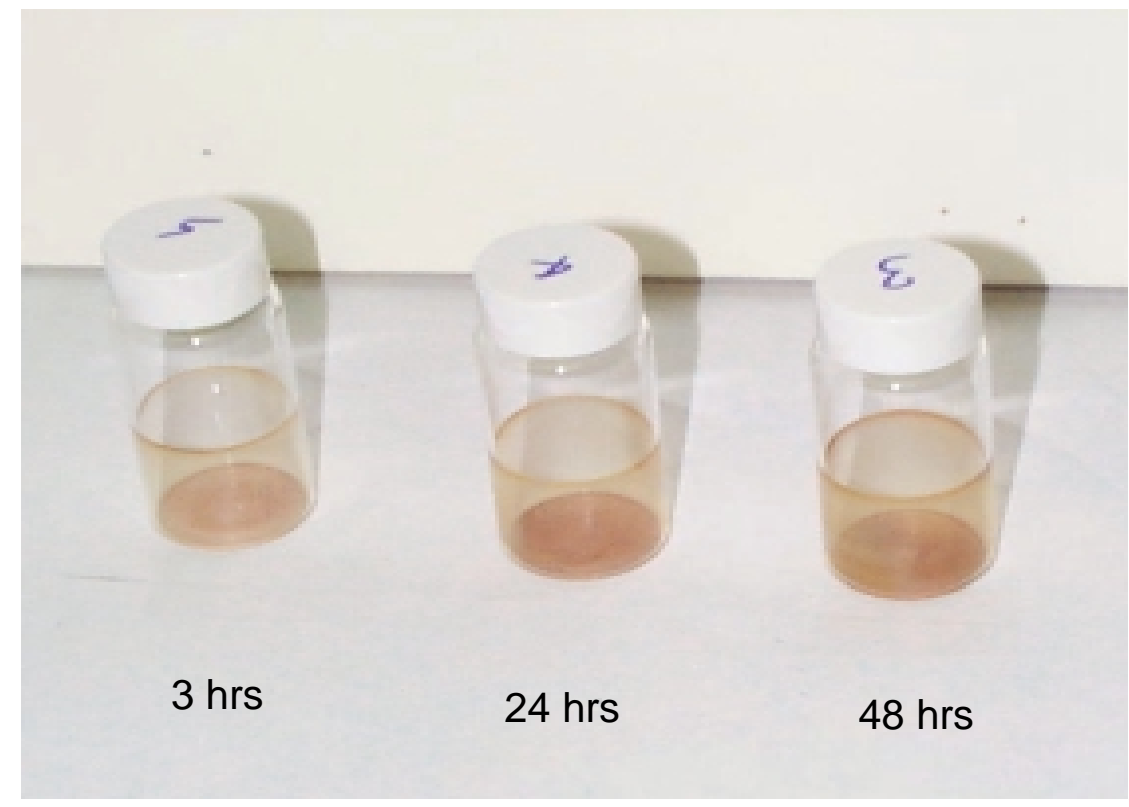

Figure 4.9 - Gum Formation in Samples Stored at $21^{\circ} \mathrm{C}$ 


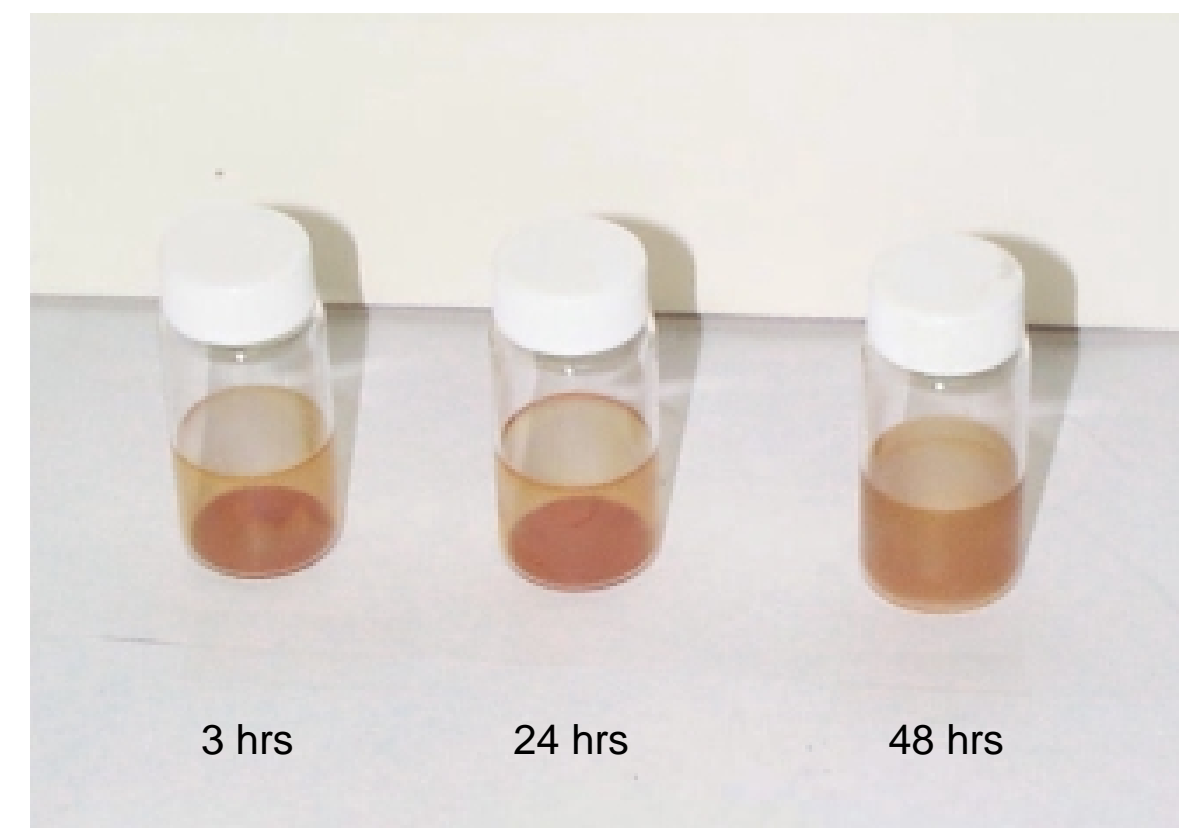

Figure 4.10 - Gum Formation in Samples Stored at $32^{\circ} \mathrm{C}$ 
The effect of temperature on the rate of gum formation can also be seen in Figure 4.11. The weight percentage of gum formed was obtained as described in Section 3.5. The gum formed increased significantly at the early stage of storage and then remained fairly constant with time. The higher the storage temperature, the faster is the rate of gum formation. The initial rates of gum formation at different storage temperatures are listed in Table 4.5. The initial rates were obtained by drawing straight lines between two data points on portions where the gum formation is increasing.

Table 4.5. Initial Rates of Gum Formation of Coal liquids at Different Storage Temperatures

\begin{tabular}{|c|c|}
\hline Temperature $\left({ }^{\circ} \mathrm{C}\right)$ & $\begin{array}{c}\text { Initial Rate of Gum Formation } \\
\text { (wt.\% / hour) }\end{array}$ \\
\hline 2 & 0.76 \\
\hline 21 & 1.47 \\
\hline 32 & 1.84 \\
\hline
\end{tabular}

Similar results were obtained by Brown and Karn [41], and Karn et al. [42] when they studied the stability of coal liquids at various storage temperatures. They also found that the rate of degradation increases as the storage temperature increases. In their study, the rate of degradation was measured by the rate of viscosity increase. This confirms the fact that storing fuels at higher temperatures is an accelerated method for fuel deterioration [23, 24]. 


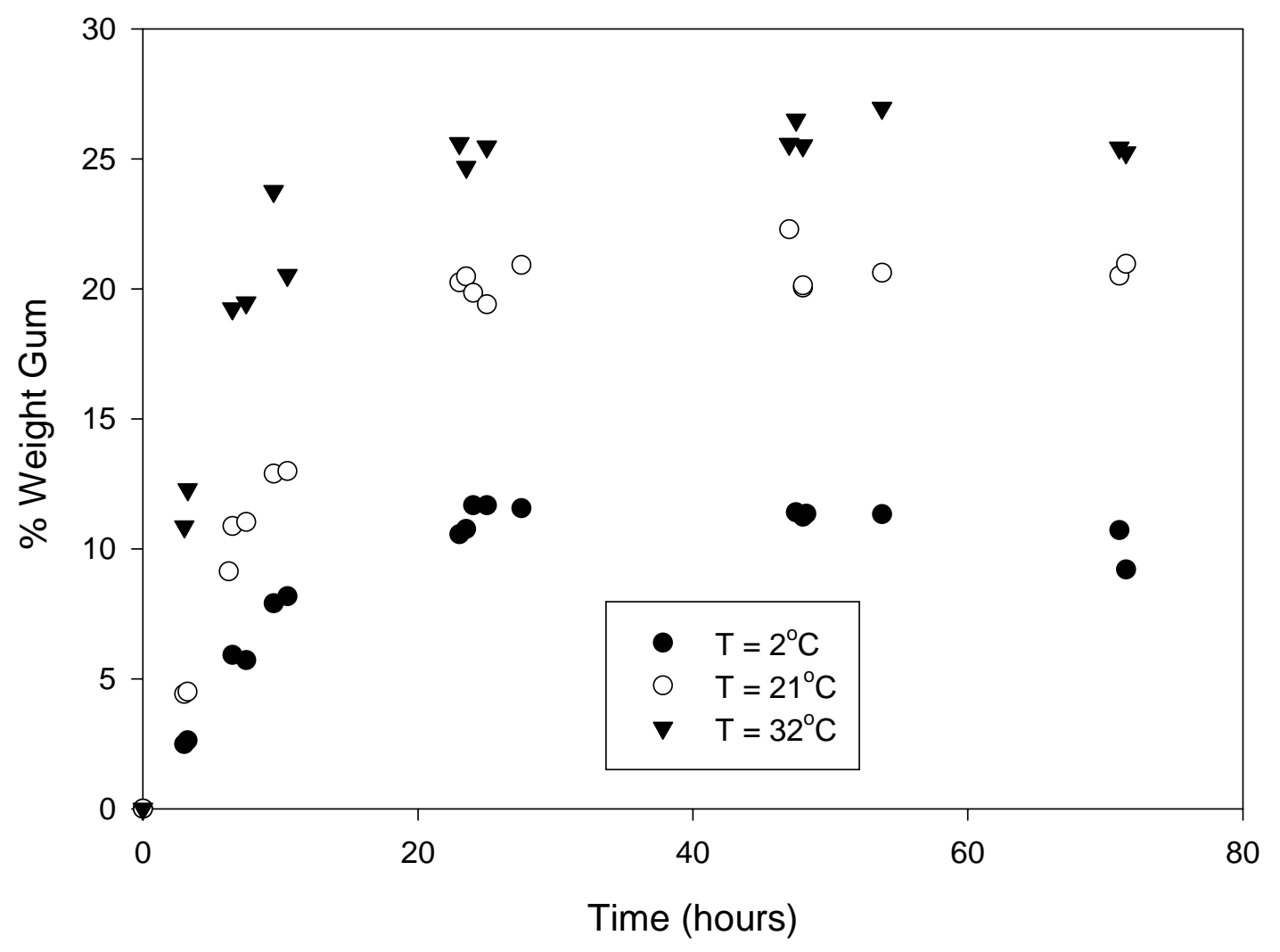

Figure 4.11 - The Effect of Temperature on the Rate of Gum Formation 
From Figure 4.11, after the rate of gum formation increased significantly at the beginning of storage, the gum remained fairly constant after 24 hours. White [63] obtained a similar trend in his fuel stability study. He examined the stability of straight run (SR) stocks, light cycle oils (LCO) and blends of SR and LCO, for up to 96 hours stored at $95^{\circ} \mathrm{C}$ while oxygen is being bubbled through the sample at a rate of 3L/h. SR stocks are distillation cuts from crude oil and LCO's are from a fluid catalytic cracking unit which consist mainly of aromatics (>50\%). The response curves of total insolubles as a function of time exhibit a tendency toward an Sshaped curve consisting of an induction period, followed by a period of rapid formation of total insolubles, and finally a leveling off to some final value. White suggested that the samples undergo an oligomerization process with a specific chemical composition and that reactive nitrogen compounds may be involved in sediment formation.

Dorbon et al. [64] also found that a large amount of insolubles is formed during the first few hours, and then the increase became slower when they studied the stability of SR and LCOs from crude oil. The samples were stored for only 40 hours at $120^{\circ} \mathrm{C}$ while pure oxygen was being bubbled through the sample at a rate of $3 \mathrm{~L} / \mathrm{h}$. They also stored the samples at $43^{\circ} \mathrm{C}$ for up to 12 weeks without bubbling oxygen during storage. The response curves show a gradual increase in insolubles followed by stabilization after 8 weeks. No explanations were given by the authors regarding the leveling off of gum formed towards the later portions of the storage period. 
Brinkman and Bowden [46] stored selected samples of syncrudes in borosilicate bottles at $43{ }^{\circ} \mathrm{C}$ for up to 32 weeks. The samples were aerated by bubbling air everyday to ensure adequate oxygen supply in the stored bottles. Exxon Donor Solvent naphthas from Illinois No. 6 and from Wyodak coals, and SRC II naphtha show sharp increase in total gum after 4 weeks. However, only a slow gradual increase in gum level was then observed for up to 32 weeks. Brinkman and Bowden suggested that heavier fractions undergo polymerization to produce gums.

The results shown in Figure 4.11 are consistent with earlier studies [46, 63, 64]. A possible explanation for this behaviour is the saturation limit discussed by White [65]. First, gum formation is initiated when the fuel sample comes in contact with an oxygen-containing atmosphere. The rate of reaction is dependent on the nature of the fuel sample and the materials with which it comes into contact. Acidic materials can catalyze some of the potential reactions. Second, some of the reaction products could be soluble in the fuel sample and only after the saturation limit is reached do the particulates begin to appear in the fuel as filterable insolubles.

During the period required to reach saturation, another rate process is already removing instability products from the fuel. It is a diffusion process of the instability products to the glassware surface. Thus, while the instability reactions are placing reaction products in the solution, the diffusion and adsorption process is removing material from the solution and depositing it on the glassware. This may explain the reason why insoluble gum always appears first at the wall of the glass 
sample vial like a thin lining as early as three hours into the storage period (see Figures 4.8, 4.9, and 4.10).

Assuming that the reaction process is faster than the rate at which reaction products are removed from solution by diffusion, the solubility limits of the reaction products in the fuel will eventually be reached and the filterable insolubles will begin to precipitate from the solution. Thus, at the saturation point, the reaction stops and the rate of gum formation levels off.

Although, the coal liquid samples used in the current study were concentrated hexane-oil solutions, the shape of the curves obtained is similar with past studies. Since these samples are hexane diluted in contrast to the neat samples from earlier work, the reduced solubility of larger, product molecules in hexane will cause gum formation to occur earlier into the storage period. Wright and Weimer [47] discovered that dilution of SRC I and SRC II liquids with methylene chloride and stored at room temperature caused similar degradation as undiluted samples stored at higher temperatures. This suggests that dilution may be another method of accelerated degradation.

\subsubsection{Gum Measurements}

As discussed in Section 4.3.1, there are several degradation reactions taking place at the same time during storage. Both soluble and insoluble products are produced from these reactions. However, in the determination of gum weight (described in Section 3.5), after the coal liquid sample was filtered, the sample vials were rinsed several times with hexane to ensure that no hexane-soluble materials remained in the sample vial. Thus, the weight of the gum reported in this study is 
limited to the hexane-insoluble products only. They are obtained gravimetrically without the use of any other analytical methods.

\subsection{HPLC Hydocarbon Compound Class Analysis}

\subsubsection{Compound Class Separations}

Compound classes were identified using both retention time data from the standards and the UV spectra measured on the diode-array detector. Table 4.6 shows the retention times of pure standards used in the analysis.

Table 4.6. Retention Times of Pure Standards for the HPLC Hydrocarbon Compound Class Analysis

\begin{tabular}{|c|c|c|c|}
\hline Compound Name & Compound Type & $\begin{array}{c}\text { UV Retention } \\
\text { Time (mins) }\end{array}$ & $\begin{array}{c}\text { ELSD Retention } \\
\text { Time (mins) }\end{array}$ \\
\hline $\mathrm{n}-\mathrm{C}_{22}$ & Saturate & $8.71^{\mathrm{a}}$ & 9.71 \\
\hline $\mathrm{C}_{19^{-}}$-Benzene & 1-Ring Aromatic & 12.72 & 13.23 \\
\hline Naphthalene & 2-Ring Aromatic & 19.16 & $20.16^{\mathrm{b}}$ \\
\hline Anthracene & 3-Ring Aromatic & 26.49 & 27.50 \\
\hline Pyrene & 4-Ring Aromatic & 29.17 & 30.20 \\
\hline Carbazole & Polar & 61.12 & 62.10 \\
\hline
\end{tabular}

${ }^{\text {a }}$ Calculated from the ELSD retention time.

${ }^{\mathrm{b}}$ Calculated from the UV retention time.

Figure 4.12 was selected as a representative three-dimensional UV spectrum plot for all the coal liquid samples tested. The separation of the 1-ring aromatic compounds from the multi-ring aromatic compounds can be clearly seen from this figure. The 1-ring aromatics elute after approximately 12 minutes and the multi-ring aromatics begin to elute after 15 minutes. Multi-ring aromatics can be identified as having 2,3 , and 4 rings by examining the UV spectra of each compound class. The 


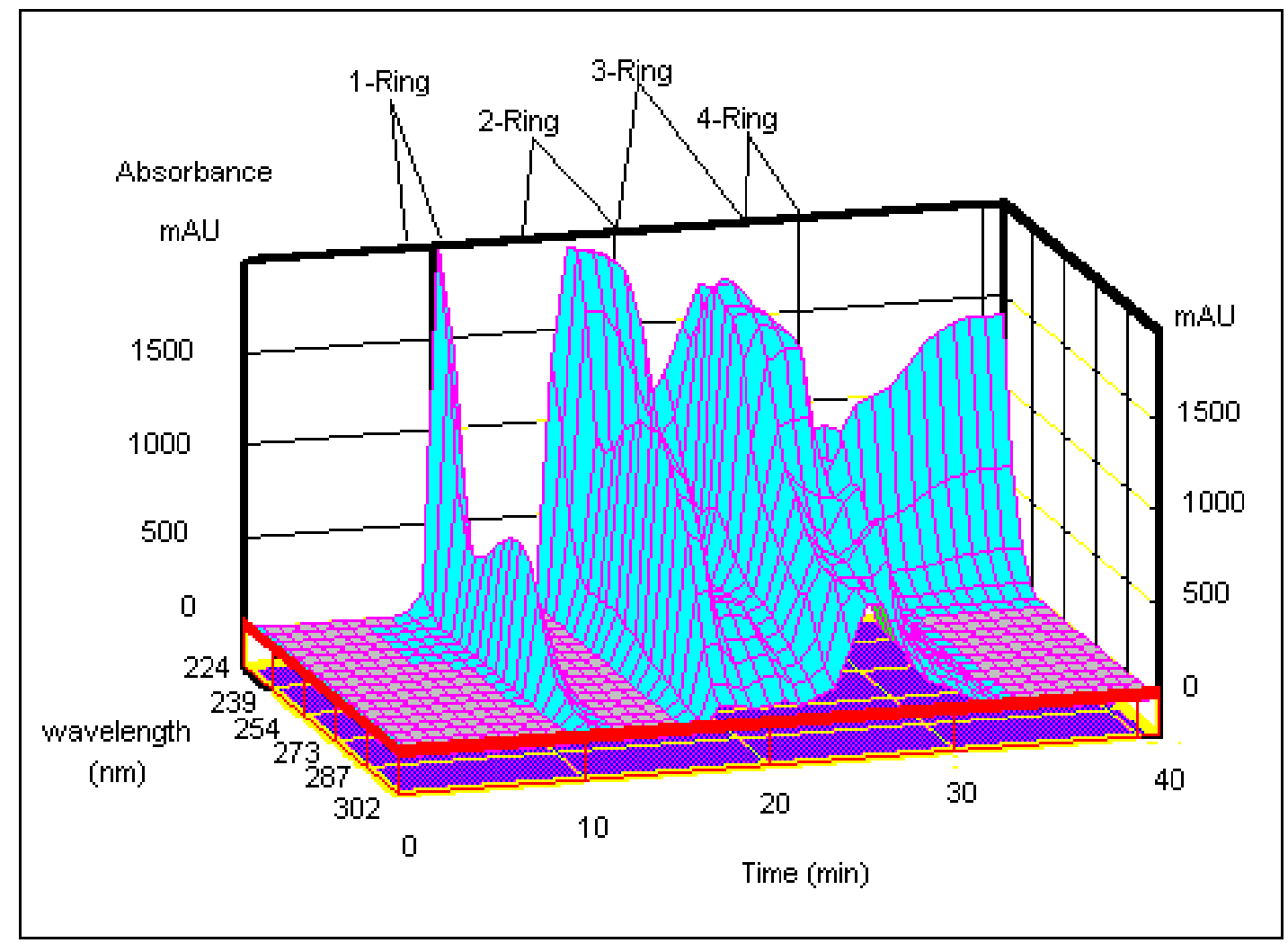

Figure 4.12 - A Three-Dimensional UV Spectrum Plot of a Fresh Coal Liquid Sample 
division between the 2- and the 3-ring aromatics is shown by an abrupt rise in absorbance at $254 \mathrm{~nm}$ for 3-ring aromatics. The 4-ring aromatics can be distinguished from the 3-ring aromatics at around $273 \mathrm{~nm}$ because of the rise in absorbance in this region from the 4-ring aromatics. The three-dimensional UV spectrum plot for the 2-, 3-, and 4-ring aromatic standards is shown in Appendix 3.

Figure 4.13 shows the absorbance plot of the same data shown in Figure 4.12 for 2-, 3- and 4-ring aromatics at $259 \mathrm{~nm}$. The retention times and the separation of various aromatic compounds present in the sample can be clearly seen in this figure. There appears to be at least two 2-ring compounds present, at least two 3-ring compounds, and at least four 4-ring compounds. Retention times for all coal liquid samples lie within the ranges shown in Table 4.6 and have spectral characteristics that are qualitatively similar to those measured for the multi-ring aromatic standards.

Figure 4.14 is a representative ELSD chromatogram of a fresh coal liquid sample. It shows the successful separation of the saturate, aromatic, and polar fractions. The separation between the saturates and 1-ring aromatics on the PAC column is consistent with the retention time data for standards shown in Table 4.6 and confirms the complete separation of saturates and 1-ring aromatics. Although the UV spectra show that there are 2-ring aromatics present in the coal liquid sample, negligible 2-ring aromatics were detected by the ELSD. This is due to the fact that only components with boiling points greater than $315^{\circ} \mathrm{C}$ can be detected by ELSD. Figure 4.14 also shows the cluster of multi-ring aromatics separated by the DNAP column. The divisions between the aromatic classes are based on the 


\section{Absorbance}

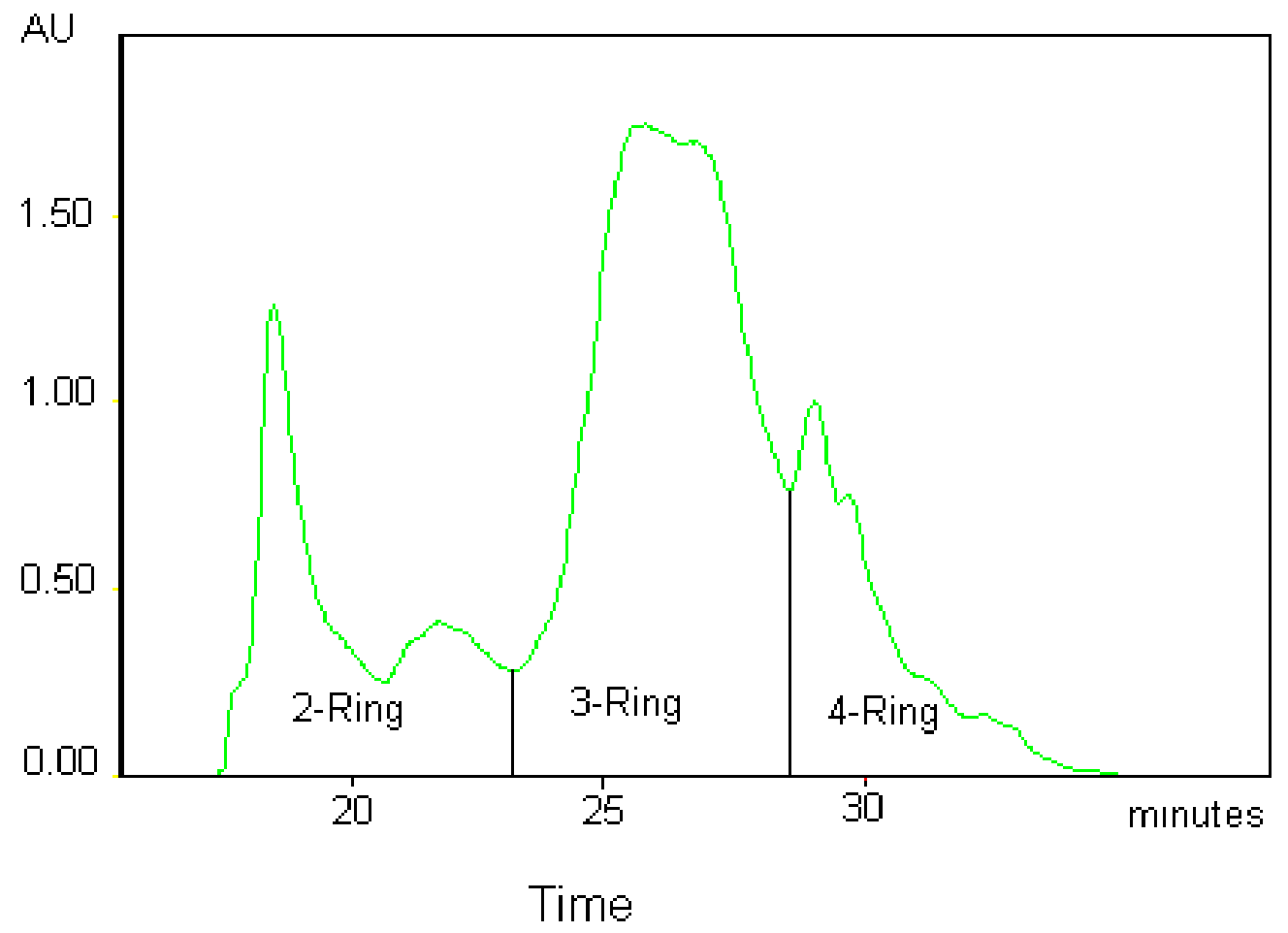

Figure 4.13 - Absorbance Plot of the Same Data Shown in Figure 4.12 for 2-, 3-, and 4-Ring Aromatics at $259 \mathrm{~nm}$ 


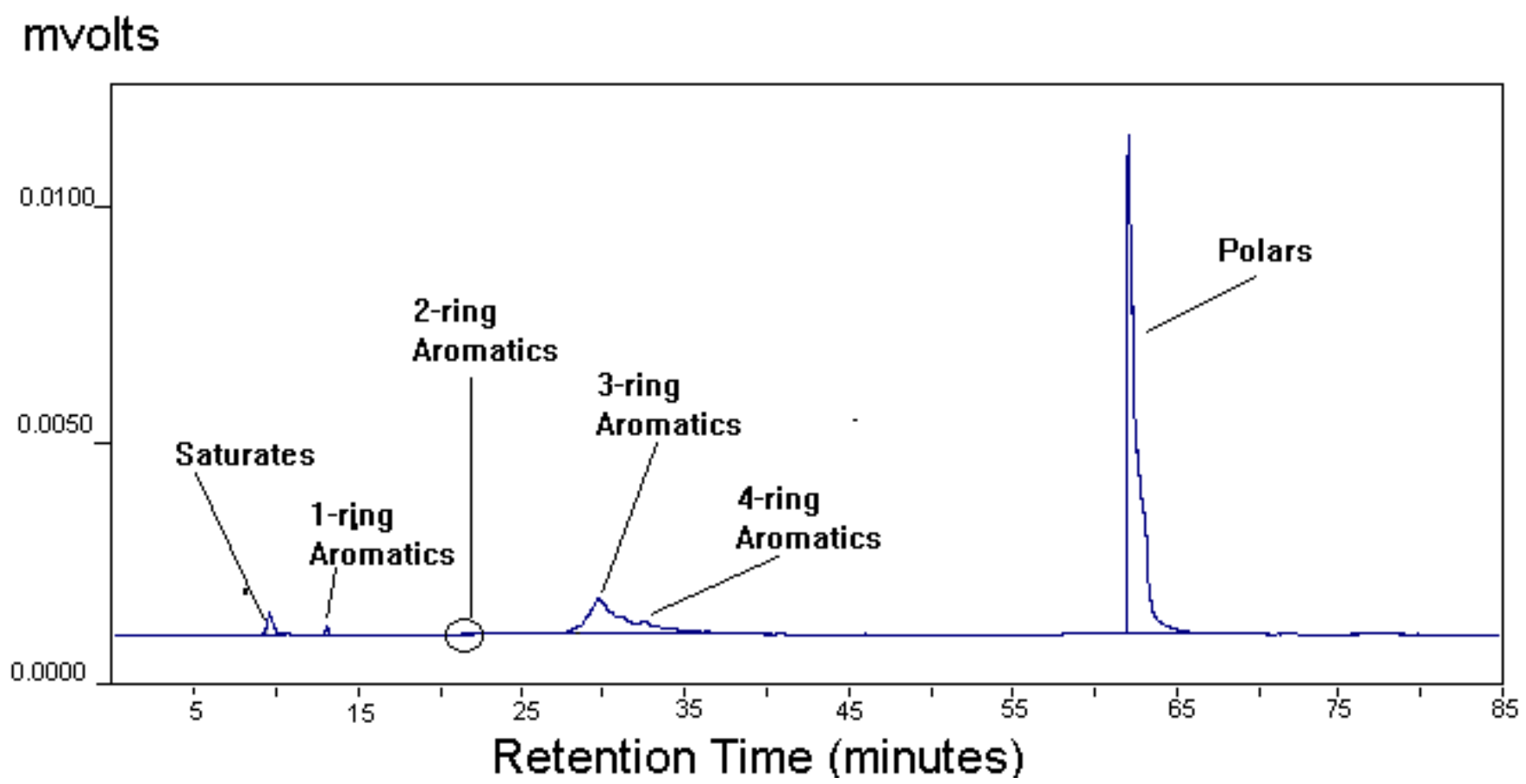

Figure 4.14 - ELSD Chromatogram of a Fresh Coal Liquid Sample 
retention times and the UV spectra of the standards. The polar compounds were separated by and eluted from the DIOL column. Information regarding these polar compounds will be discussed in Section 4.7.

\subsubsection{Changes in Coal Liquid Composition}

For a fresh coal liquid sample, the percentage weight composition typically ranges from $1 \%$ to $5 \%$ for the saturates, $40 \%$ to $47 \%$ for the aromatics, and $34 \%$ to $40 \%$ for the polar compounds. The weight percentages for each compound class were calculated as described in Section 3.6. Figures $4.15-4.17$ are representative trends of the changes in composition for all coal liquid samples tested.

Figure 4.15 shows that the amount of saturates remained constant with time. Moreover, there were no noticeable changes in percent saturates stored at different temperatures. Based on this result, it can be concluded that the presence of saturated compounds has no effect on the formation of gum. This conclusion is also supported by Fathoni and Batts [1] who cited that paraffins are the most stable with respect to hydrocarbon composition compared to aromatics, olefins, and diolefins. Allbright et al. [30] also found that saturates are the least reactive among the several compound classes they studied. They used radioactive tracer techniques to determine the types of compounds that cause gum formation in gasoline during storage.

Both the aromatic and polar compound classes appear to be unstable. Figures 4.16 and 4.17 show that these fractions decreased significantly at the early stage of storage and then remained fairly constant with time. The decrease in aromatic compounds appears the same for all storage temperatures (Figure 4.16). 


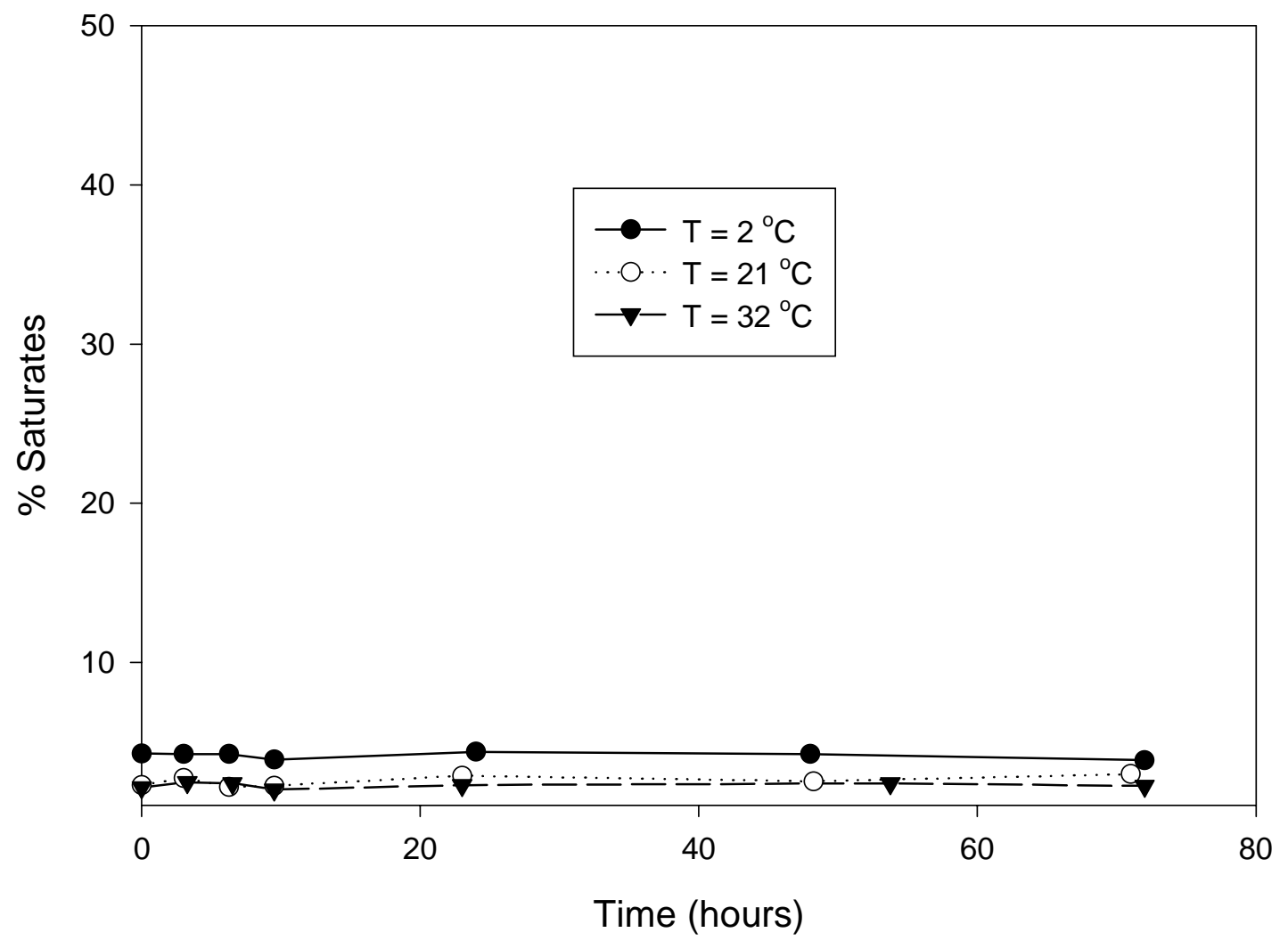

Figure 4.15 - Changes in Weight Percentage Saturate Compound Class Stored at 2, $21,32{ }^{\circ} \mathrm{C}$ (Data Obtained from ELSD) 


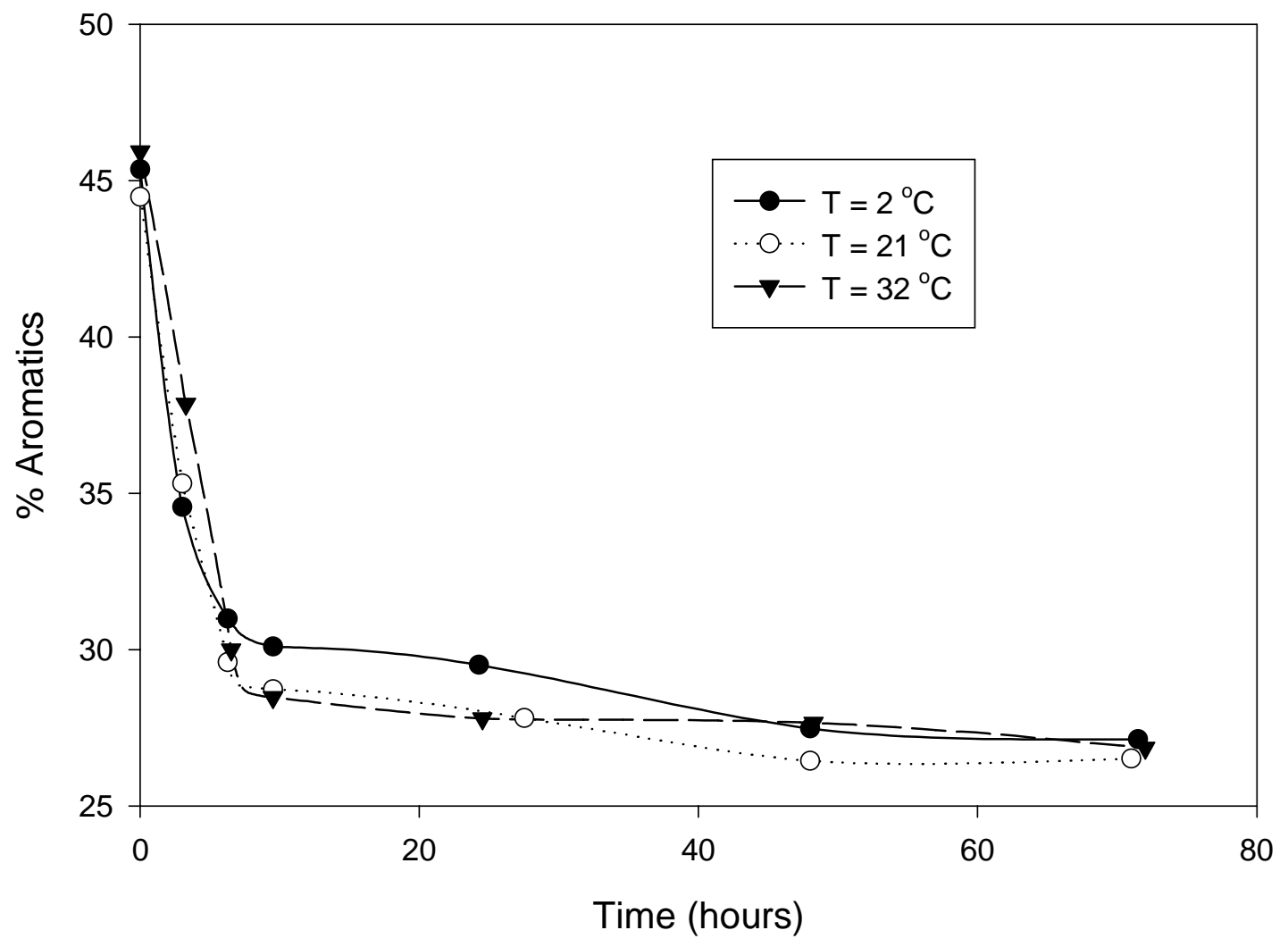

Figure 4.16 - Changes in Weight Percentage Aromatic Compound Class Stored at 2, $21,32{ }^{\circ} \mathrm{C}$ (Data Obtained from ELSD) 


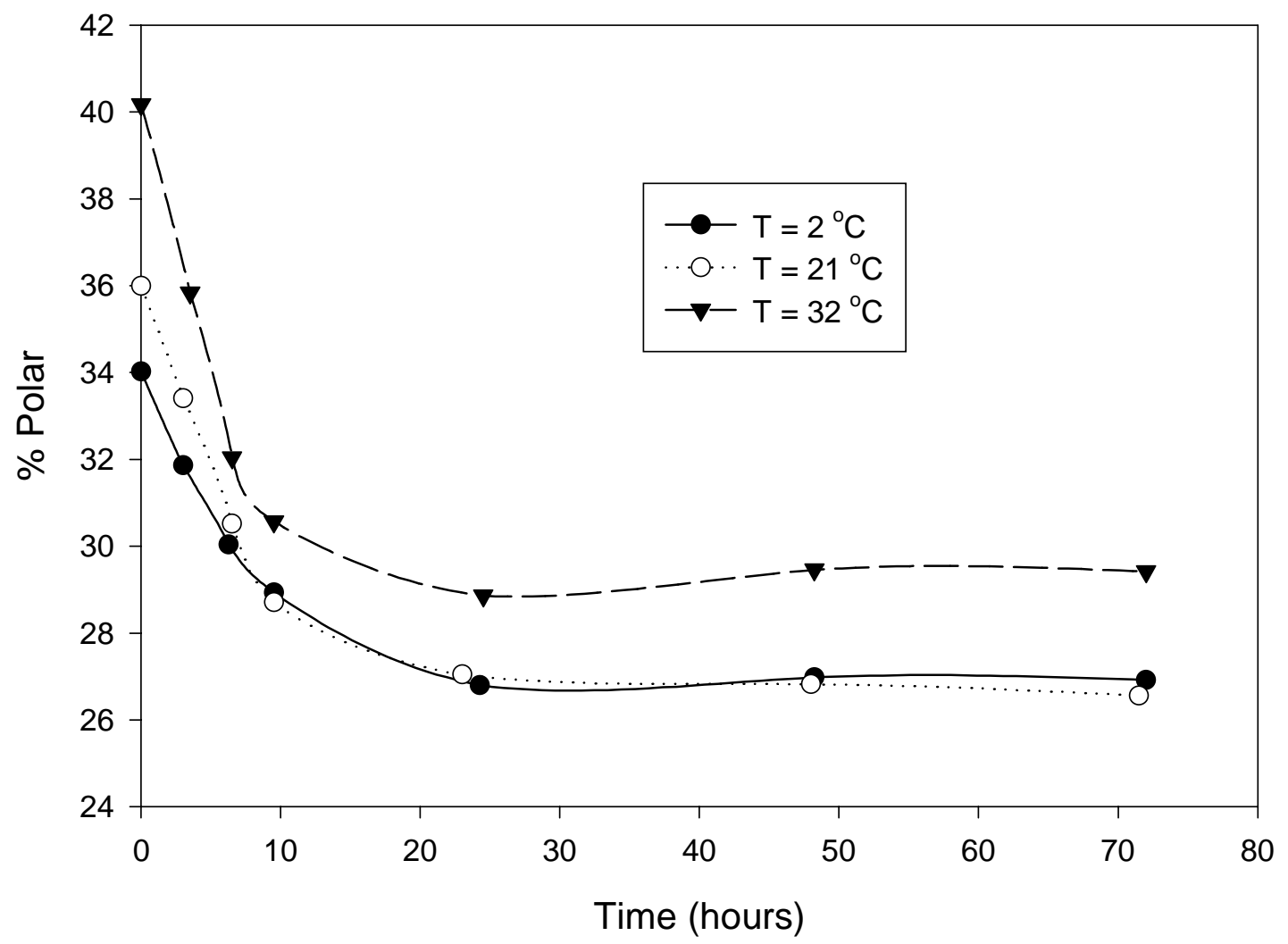

Figure 4.17 - Changes in Weight Percentage Polar Compound Class Stored at 2, $21,32{ }^{\circ} \mathrm{C}$ (Data Obtained from ELSD) 
Figure 4.17 shows that the decrease in polar compounds is fastest at highest storage temperature and slowest at lowest storage temperature.

Figures 4.18 - 4.20 show two superimposed chromatograms from an aged (72 hours) and unaged sample of coal liquid. It is clearly seen in Figure 4.18 that the peak of the saturate compound class did not change. However, the 1-ring aromatics slightly decreased. Figures 4.19 and 4.20 show that the multi-ring aromatics and polar compounds decreased significantly after 72 hours.

Ward and Schwartz [28] found that the olefinic and aromatic portions are the most unstable fractions when they studied the stability of unleaded gasolines using UV irradiation as a means of accelerating gum formation. In their continuing study, Ward and Schwartz [29] discovered that the types of aromatics that reacted to form gum, in decreasing order of reactivity, were: alkyl benzenes, tetralins and indans, and naphthalenes. Allbright et al. [30] also found that mono-olefins and alkyl benzenes are the most unstable in their study of gum formation in gasoline during storage.

The trends obtained in the current study for both the aromatic and polar compounds can be correlated with the increase in gum formation early into the storage period. If gum formation of coal liquids on aging is due to chemical reactions such as polymerization of unsaturated hydrocarbons and some reactive polar compounds, then this should be reflected in the significant decrease in aromatic and polar compound classes. This suggests that the aromatic and polar compound classes are the main reactants in the gum formation of coal liquids. 


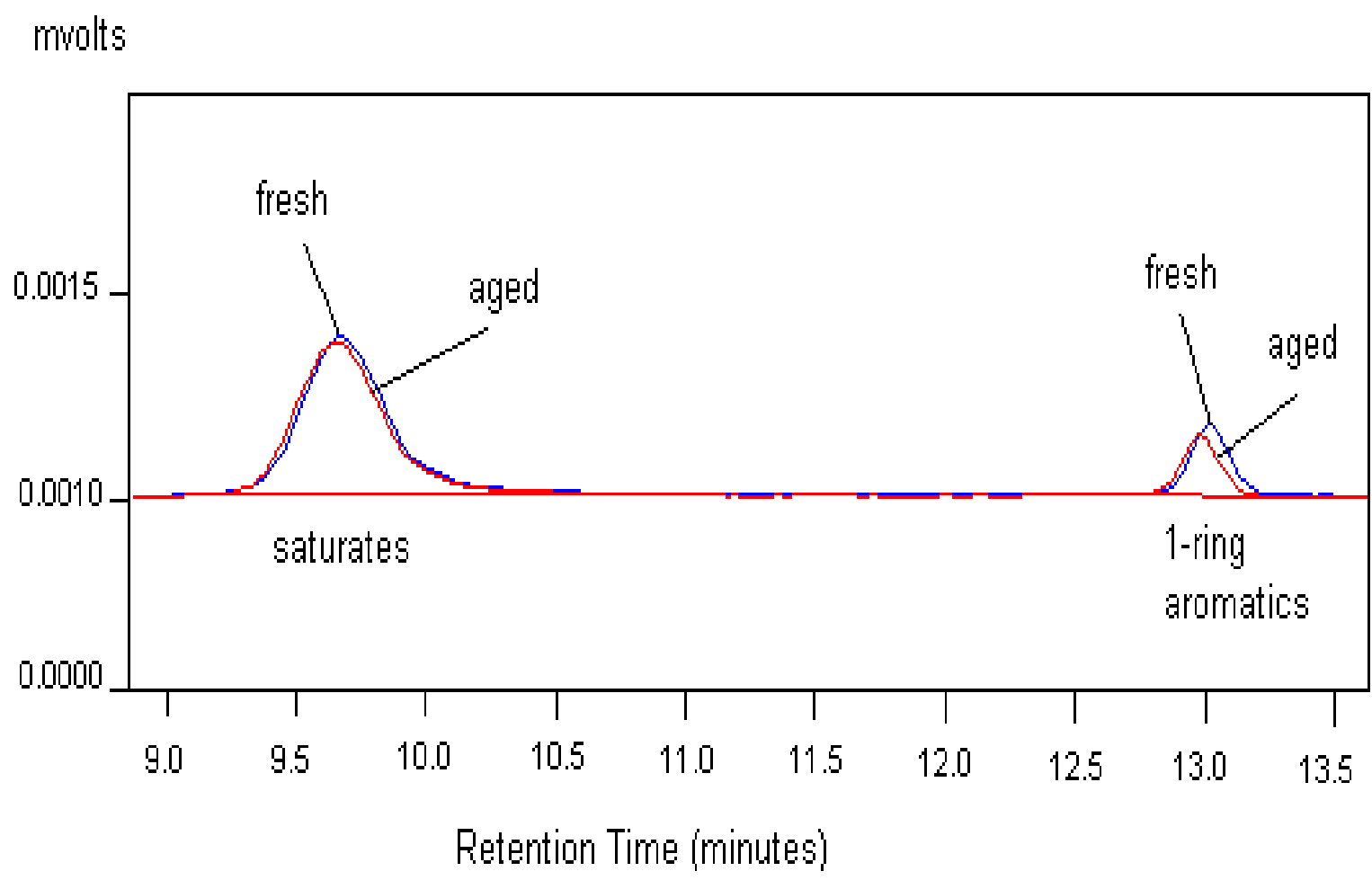

Figure 4.18 - ELSD Chromatogram of Fresh and Aged (72 hours) Coal Liquids Showing the Changes in Saturates and 1-Ring Aromatics 


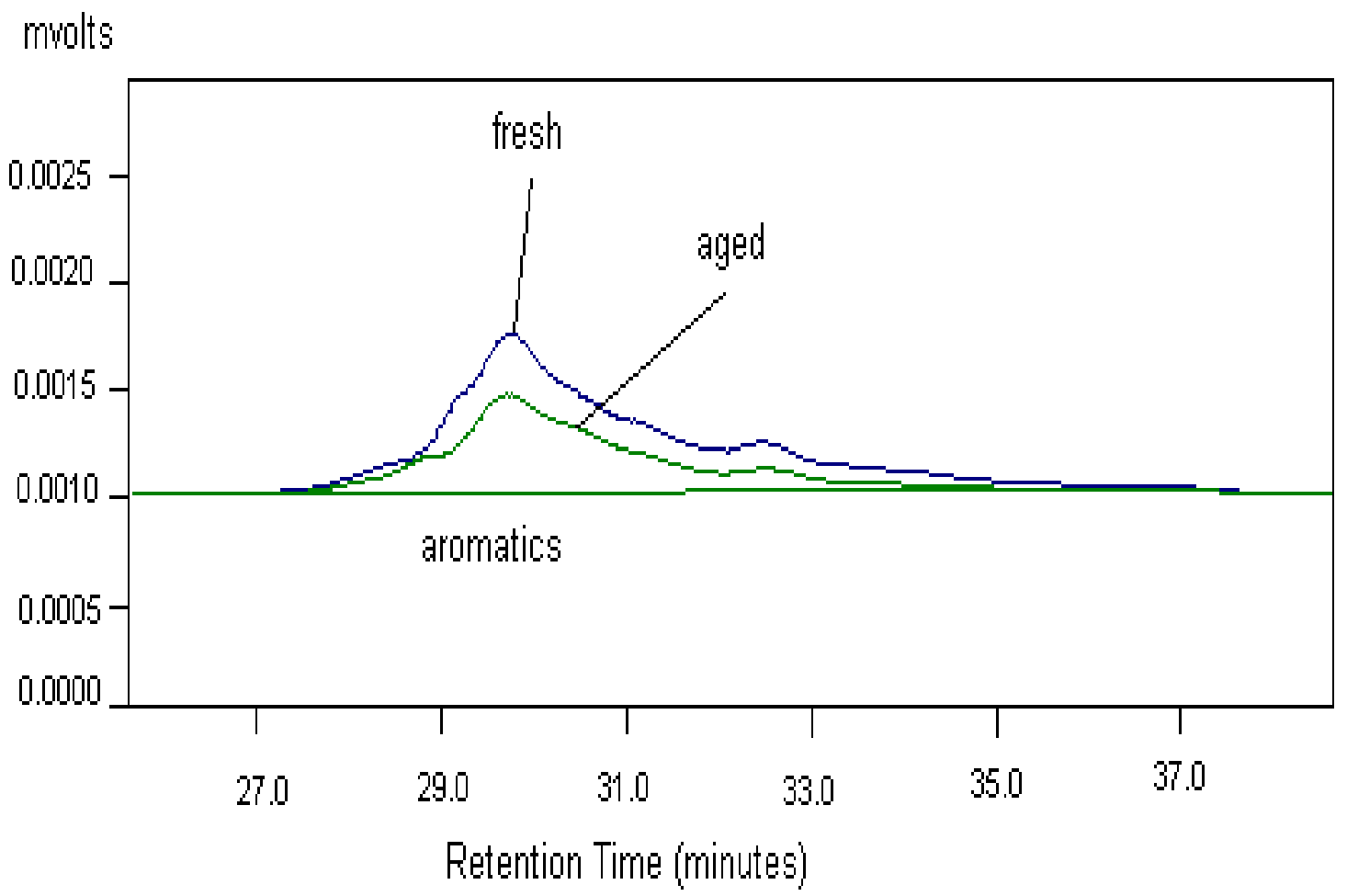

Figure 4.19 - ELSD Chromatogram of Fresh and Aged (72 hours) Coal Liquids Showing the Changes in Multi-Ring Aromatics 


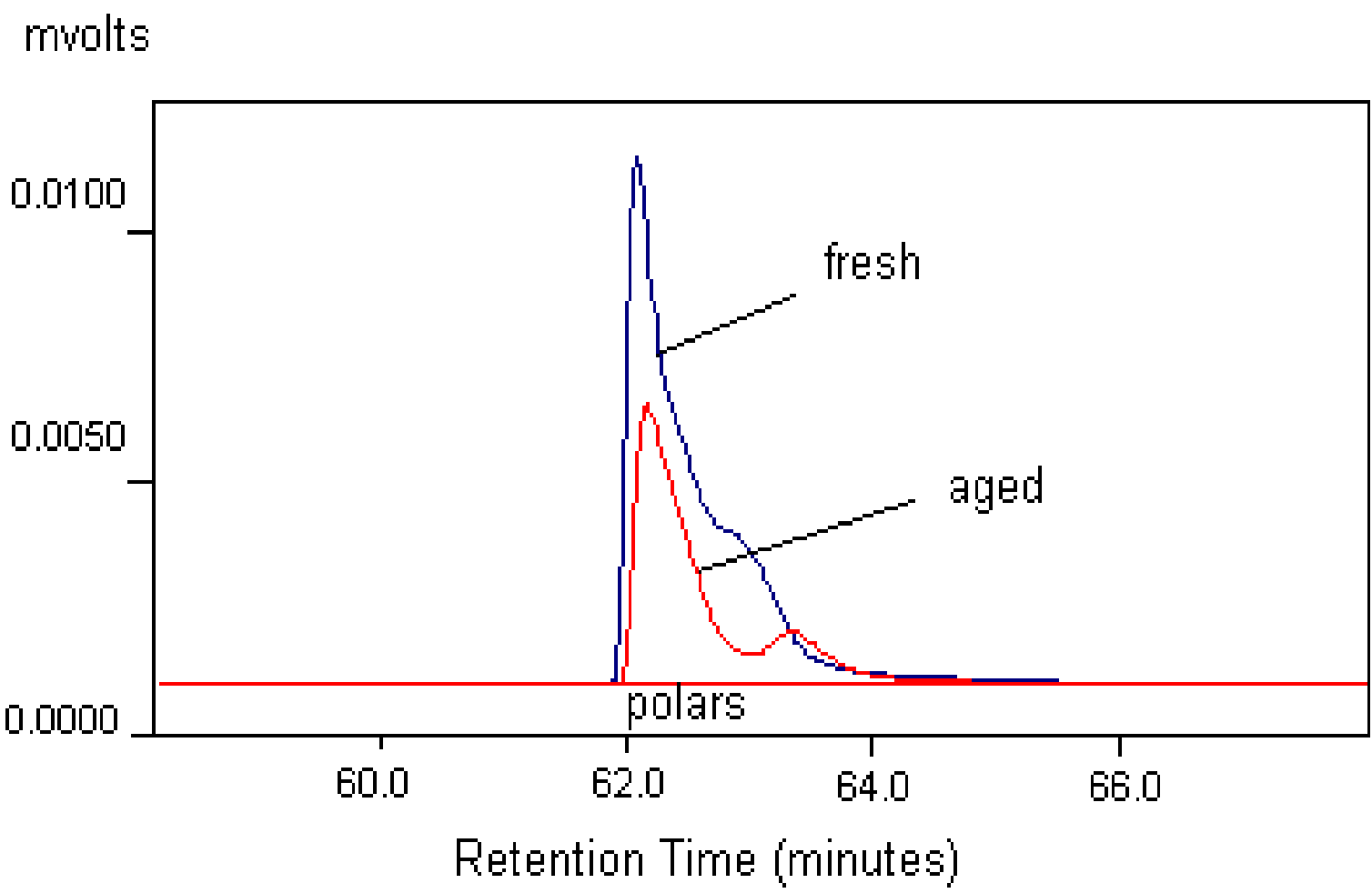

Figure 4.20 - ELSD Chromatogram of Fresh and Aged (72 hours) Coal Liquids Showing the Changes in Polar Compounds 
Bhan et al. [66] arrived at a similar conclusion when they investigated the aging of several marine diesel fuels at $65^{\circ} \mathrm{C}$. Aged and unaged fuel samples were chromatographically separated and analyzed by infrared spectroscopy, nonaqueous titration, GC/MS, and magnetic scan mass spectrometry. They found that there was considerable loss of polar compounds to sediment formation. Within the compound class, the more aromatic, higher-molecular-weight members were observed to be the most active in sediment formation.

\subsection{Simulated Distillation by ELSD}

The changes in boiling point distribution of the coal liquid samples were monitored using simulated distillation by ELSD. As discussed in Section 3.1, the goal of the simulated distillation using ELSD was to measure the amount of coal liquid that has a boiling point greater than the boiling point detection limit. When the detector temperature is set at $40{ }^{\circ} \mathrm{C}, 80{ }^{\circ} \mathrm{C}, 115{ }^{\circ} \mathrm{C}$, and $150{ }^{\circ} \mathrm{C}$, sample components with a normal boiling point greater than $315^{\circ} \mathrm{C}, 380^{\circ} \mathrm{C}, 435^{\circ} \mathrm{C}$, and $482^{\circ} \mathrm{C}$, respectively, are detected.

Figure 4.21 shows the typical ELSD chromatogram obtained from this analysis. The chromatogram has only one peak since the method does not use any columns for separation. The weight percentage of components having a normal boiling point greater than the boiling point detection limit was obtained as described in Section 3.1. The amount of components in a particular boiling point cut, say components with boiling point between $315{ }^{\circ} \mathrm{C}$ and $380^{\circ} \mathrm{C}$, was calculated by determining the difference between the amount of components with boiling point greater than $315^{\circ} \mathrm{C}$ and $380^{\circ} \mathrm{C}\left(\mathrm{BP}>315^{\circ} \mathrm{C}\right.$ minus $\left.\mathrm{BP}>380^{\circ} \mathrm{C}\right)$. The typical 


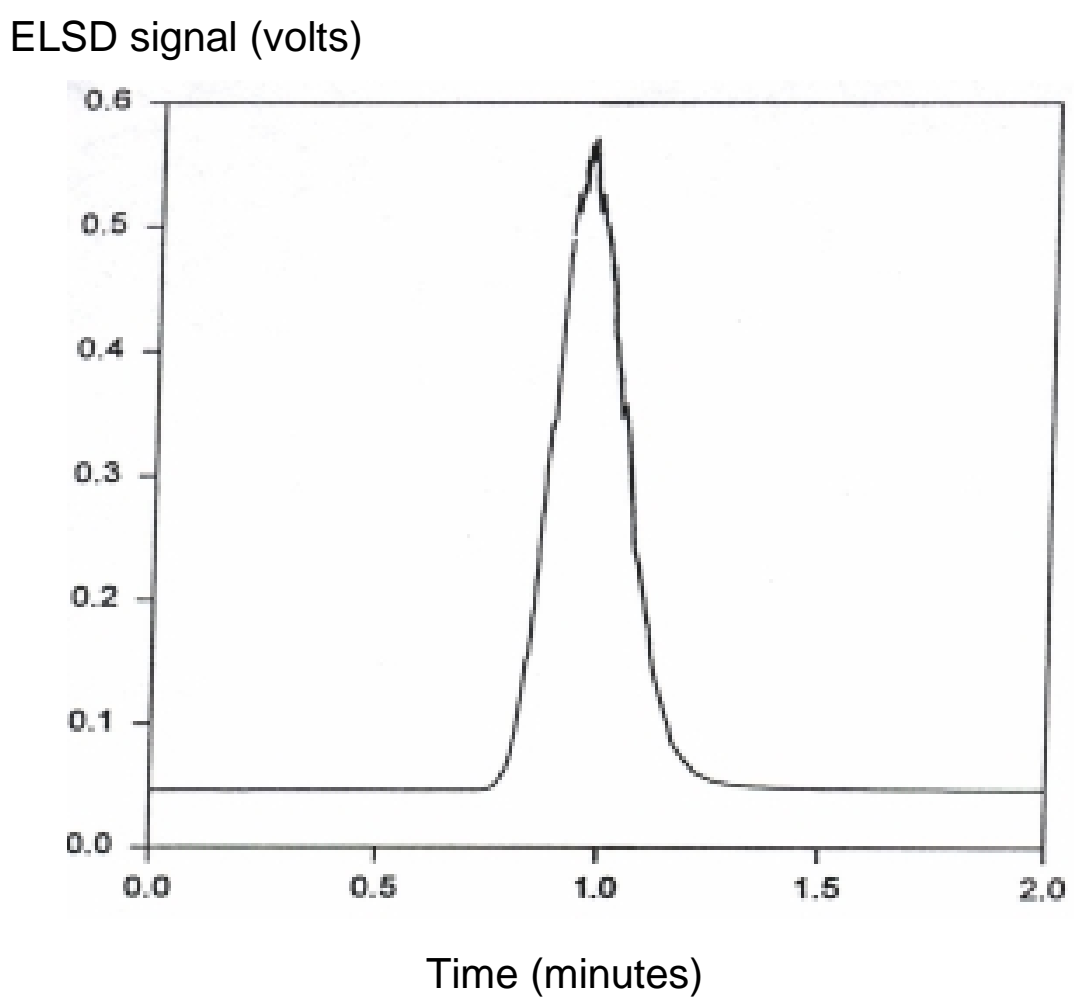

Figure 4.21 - A Typical ELSD Chromatogram Obtained from the Simulated Distillation Method 
boiling point distribution for a fresh coal liquid sample is listed in Table 4.7.

Table 4.7. Typical Boiling Point Distribution Range of a Fresh Coal Liquid Sample

\begin{tabular}{|c|c|}
\hline Boiling Point Distribution & \% Weight \\
\hline $\mathrm{BP}<315^{\circ} \mathrm{C}$ & $10-20$ \\
\hline $315^{\circ} \mathrm{C}>\mathrm{BP}>380^{\circ} \mathrm{C}$ & $20-25$ \\
\hline $380^{\circ} \mathrm{C}>\mathrm{BP}>435^{\circ} \mathrm{C}$ & $23-27$ \\
\hline $435^{\circ} \mathrm{C}>\mathrm{BP}>482^{\circ} \mathrm{C}$ & $9-15$ \\
\hline $\mathrm{BP}>482^{\circ} \mathrm{C}$ & $20-27$ \\
\hline
\end{tabular}

Figure 4.22 shows that the amount of coal liquid with boiling point between $315^{\circ} \mathrm{C}$ and $380^{\circ} \mathrm{C}$ decreases with time for all storage temperatures. Moreover, the decrease in percent coal liquid is fastest at the highest storage temperature and slowest at the lowest storage temperature. For percent coal liquid with boiling point between $380^{\circ} \mathrm{C}$ and $435^{\circ} \mathrm{C}$, the samples stored at $21^{\circ} \mathrm{C}$ and $32^{\circ} \mathrm{C}$ decreased at a faster rate compared to that stored at $2{ }^{\circ} \mathrm{C}$ (Figure 4.23). A similar trend is observed for the boiling point range between $435^{\circ} \mathrm{C}$ and $482{ }^{\circ} \mathrm{C}$ (Figure 4.24). There were no significant changes observed for percent coal liquid with boiling point greater than $482{ }^{\circ} \mathrm{C}$ stored at $2{ }^{\circ} \mathrm{C}$ (Figure 4.25). However, samples stored at 21 ${ }^{\circ} \mathrm{C}$ and $32{ }^{\circ} \mathrm{C}$ still decreased significantly at the early stage of storage. The formation of high MW species should have appeared on components with boiling point greater than $482{ }^{\circ} \mathrm{C}$ but are precipitated from the solution instead.

Storage of coal liquids at $2{ }^{\circ} \mathrm{C}$ with boiling point greater than $380^{\circ} \mathrm{C}$ had little or insignificant changes. This is also reflected by the small amount of gum formed during storage. But the significant decrease in percent coal liquid stored at $21^{\circ} \mathrm{C}$ 


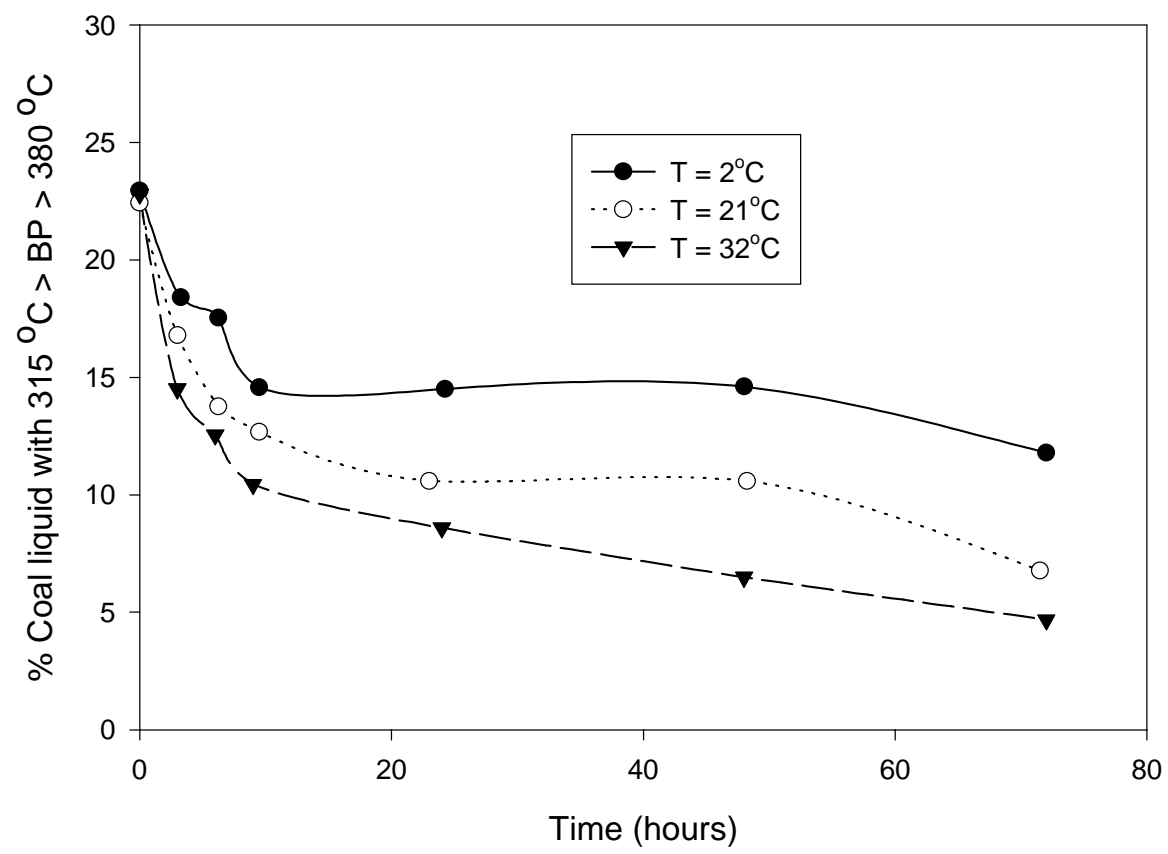

Figure 4.22 - Changes in Weight Percent Coal Liquid from ELSD with Boiling Point Between $315^{\circ} \mathrm{C}$ and $380^{\circ} \mathrm{C}$ Stored at Different Temperatures 


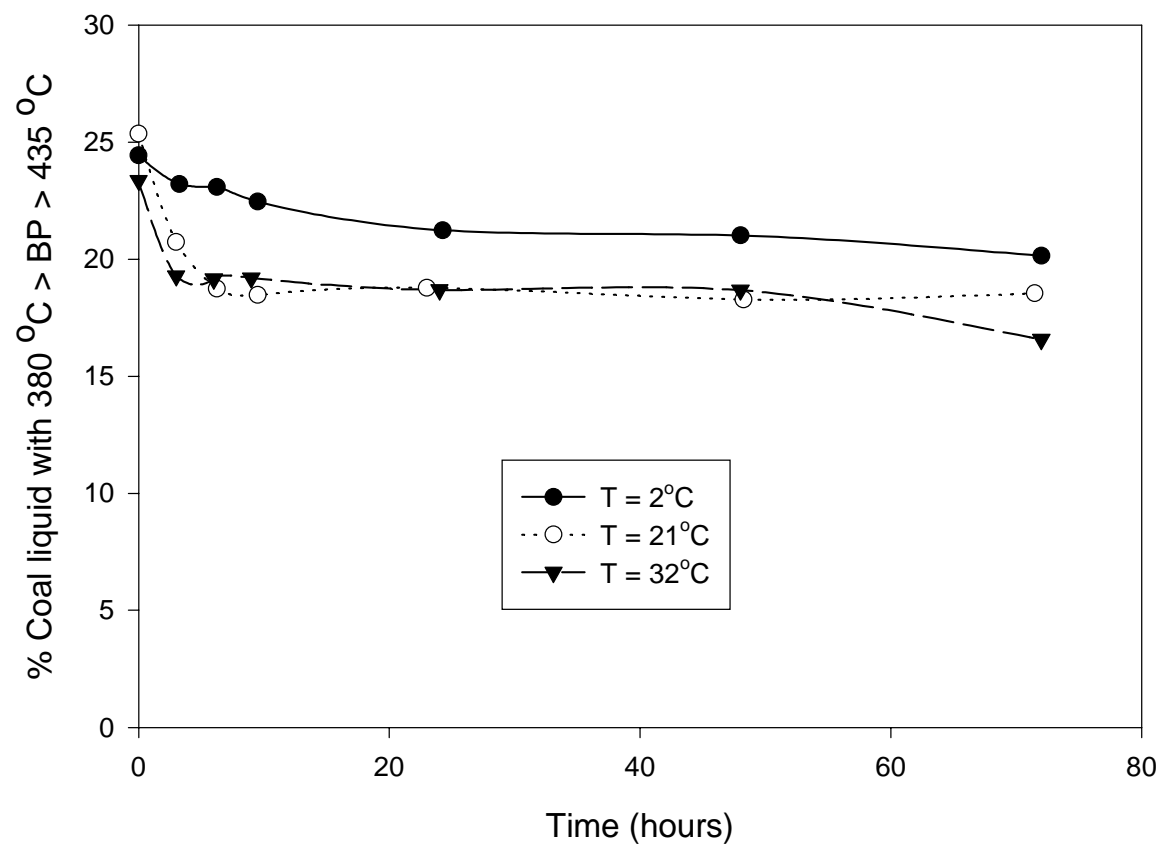

Figure 4.23 - Changes in Weight Percent Coal Liquid from ELSD with Boiling Point Between $380^{\circ} \mathrm{C}$ and $435^{\circ} \mathrm{C}$ Stored at Different Temperatures 


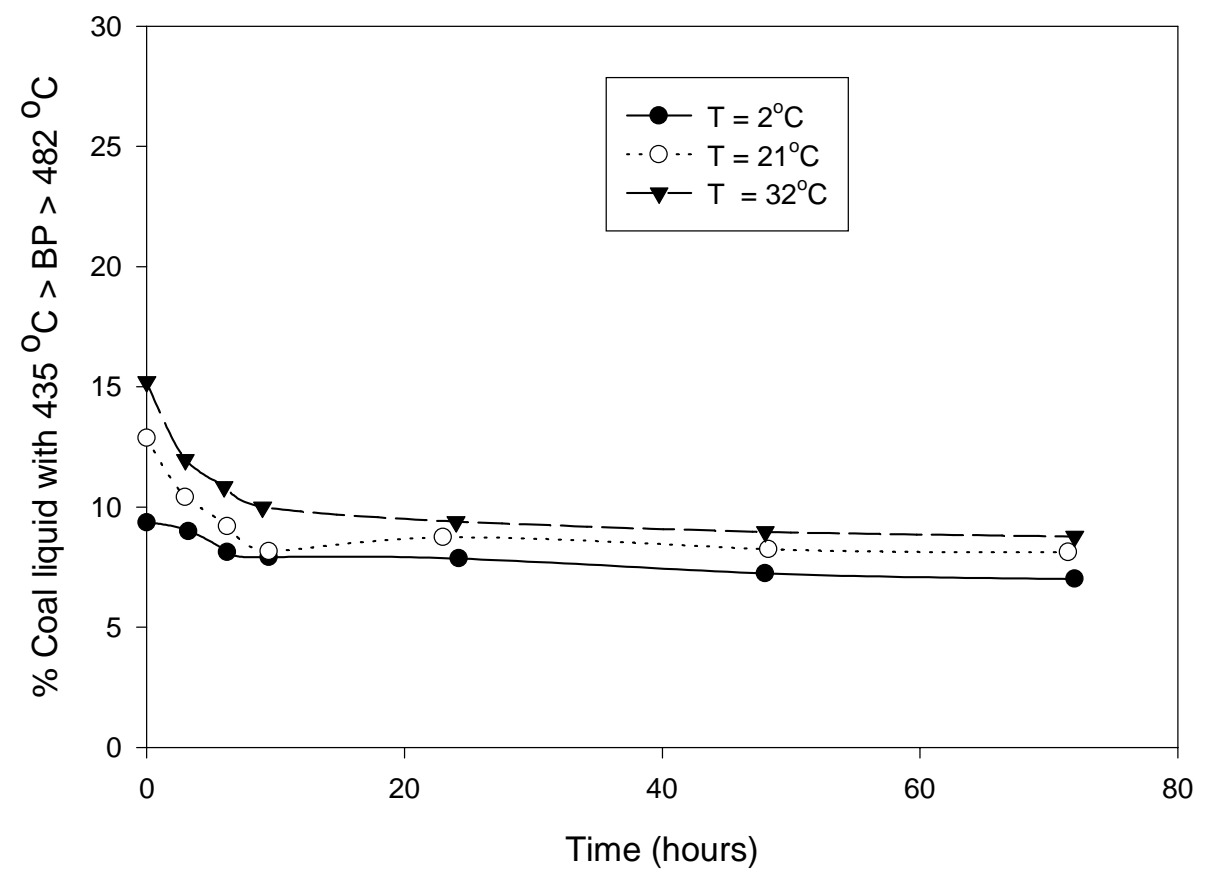

Figure 4.24 - Changes in Weight Percent Coal Liquid from ELSD with Boiling Point Between $435^{\circ} \mathrm{C}$ and $482^{\circ} \mathrm{C}$ Stored at Different Temperatures 


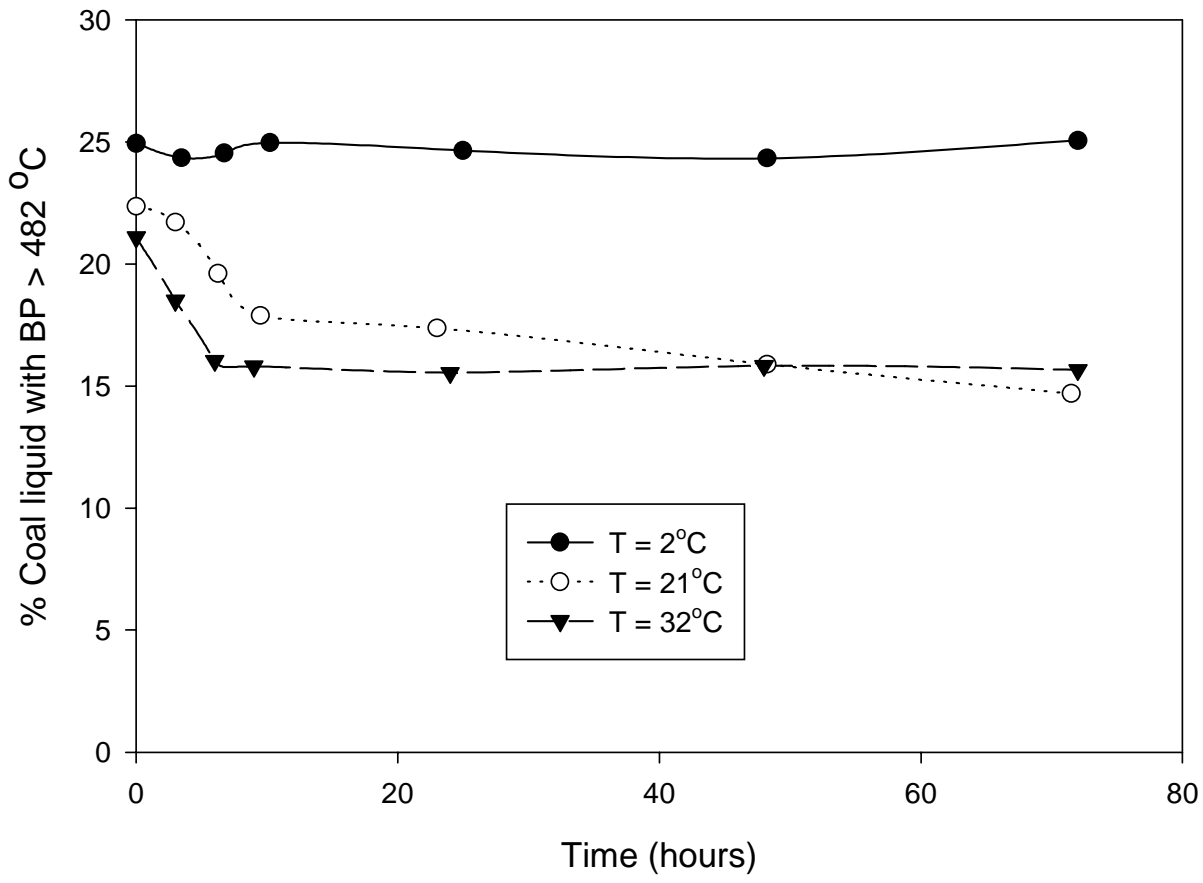

Figure 4.25 - Changes in Weight Percent Coal Liquid from ELSD with Boiling Point Greater than $482^{\circ} \mathrm{C}$ Stored at Different Temperatures 
and $32{ }^{\circ} \mathrm{C}$ with boiling point greater than $315^{\circ} \mathrm{C}$ suggests that the components are lost to the formation of higher-boiling, higher-molecular-mass compounds in the form of insoluble gum. This is reflected on the relatively larger amount of gum formed during storage at $21^{\circ} \mathrm{C}$ and $32^{\circ} \mathrm{C}$.

A similar result was obtained by Dorbon et al. [64] who studied the stability of light cycle oils. They found that the most-changing compounds were located in the heaviest distillation fraction of the LCO's with boiling point greater than $315^{\circ} \mathrm{C}$. They suggested that better stability could be achieved by removing this fraction of the LCO's. Brinkman and Bowden [46] also suggested that heavier fractions of coal derived liquids from the SRC-II process seemed to undergo polymerization to produce gums.

\subsection{Argon Gas Experiment}

\subsubsection{The Effect of Air}

An experiment was conducted to determine the effect of air on the stability of coal liquids. The procedure described in Section 3.3 was used to obtain the coal liquid samples. However, after filtration, the sample vials with the coal liquids were opened and stored in a controlled argon-atmosphere glove box at room temperature $\left(21^{\circ} \mathrm{C}\right)$ under fluorescent light. Prior to this, the glove box was purged and filled with argon gas. 
Table 4.8 shows the average results from the Hydrocarbon Compound Class Analysis and the weight percent of gum produced during each storage time. Results show that the saturates remained constant in the absence of air. Figure 4.26 shows a comparison of weight percent saturates for samples stored with and without air.

Table 4.8. Results from the Hydrocarbon Compound Class Analysis and Weight Percent of Gum Produced in the Absence of Air

\begin{tabular}{|c|c|c|c|c|}
\hline Time (hours) & \% Saturates & \% Aromatics & \% Polars & \% Gum \\
\hline 0 & 4.65 & 39.42 & 43.00 & - \\
\hline 3 & 4.42 & 38.65 & 41.05 & 6.28 \\
\hline 6 & 4.70 & 37.95 & 41.36 & 4.76 \\
\hline 9.75 & 4.52 & 36.50 & 40.06 & 5.65 \\
\hline 22.75 & 3.43 & 35.40 & 39.62 & 7.36 \\
\hline 48.5 & 3.76 & 34.20 & 38.00 & 8.59 \\
\hline 72.5 & 4.06 & 33.20 & 36.43 & 10.22 \\
\hline
\end{tabular}

Both the aromatic and the polar compounds decreased gradually in the absence of air. These results can be compared with the ones stored at $21^{\circ} \mathrm{C}$ with air. Figures 4.27 and 4.28 show the comparison of percent aromatics and percent polar compounds stored at $21^{\circ} \mathrm{C}$ with and without the presence air. There were no abrupt decreases in percent aromatics and percent polar compounds for samples stored without air. The rate of decrease is slower compared to samples stored with air. These results suggest that the absence of oxygen slows down the rate of loss of aromatics and polar compunds to sediment formation. 


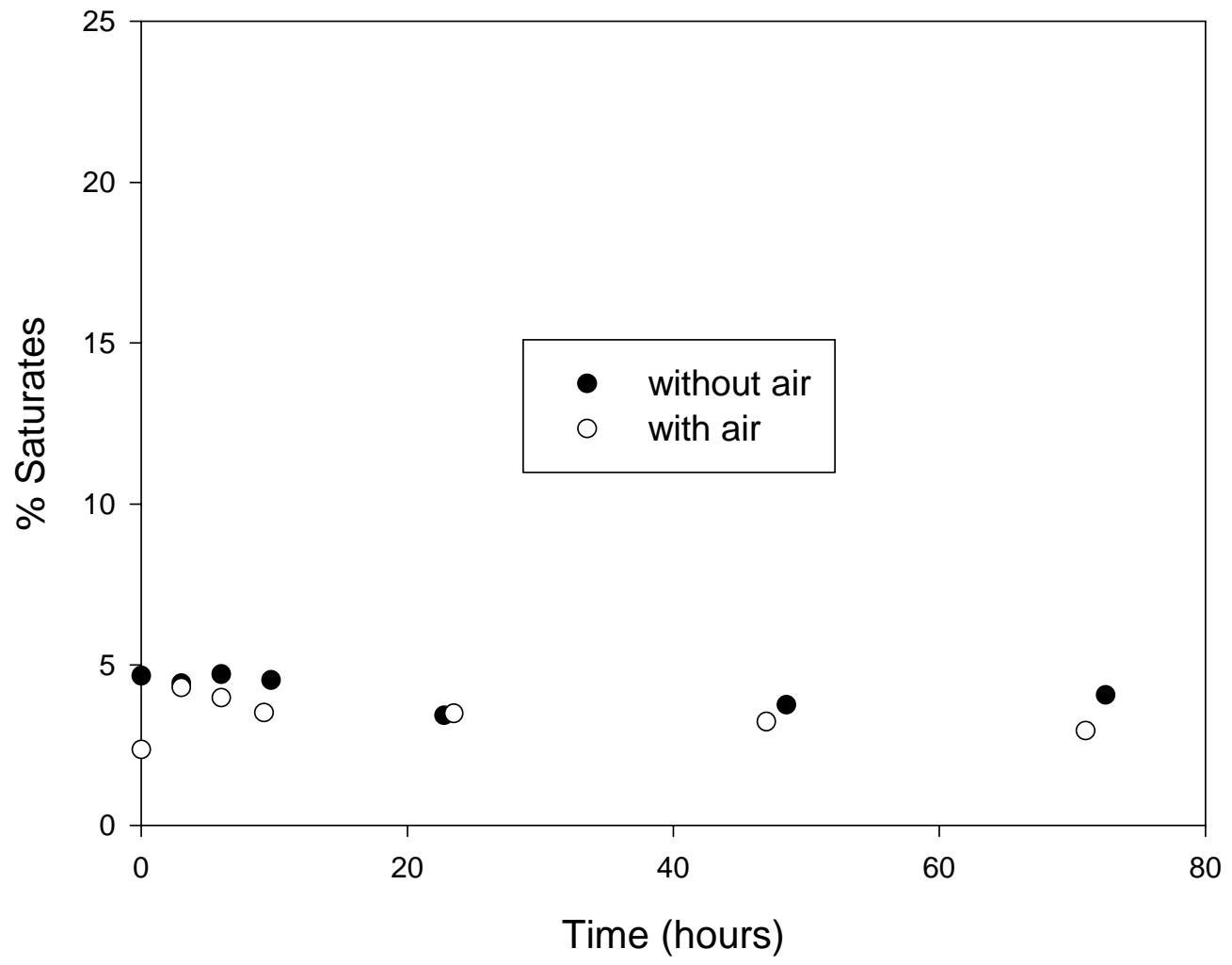

Figure 4.26 - Comparison of Weight Percent Saturates Stored at $21^{\circ} \mathrm{C}$ With and Without Air (Data Obtained from ELSD) 


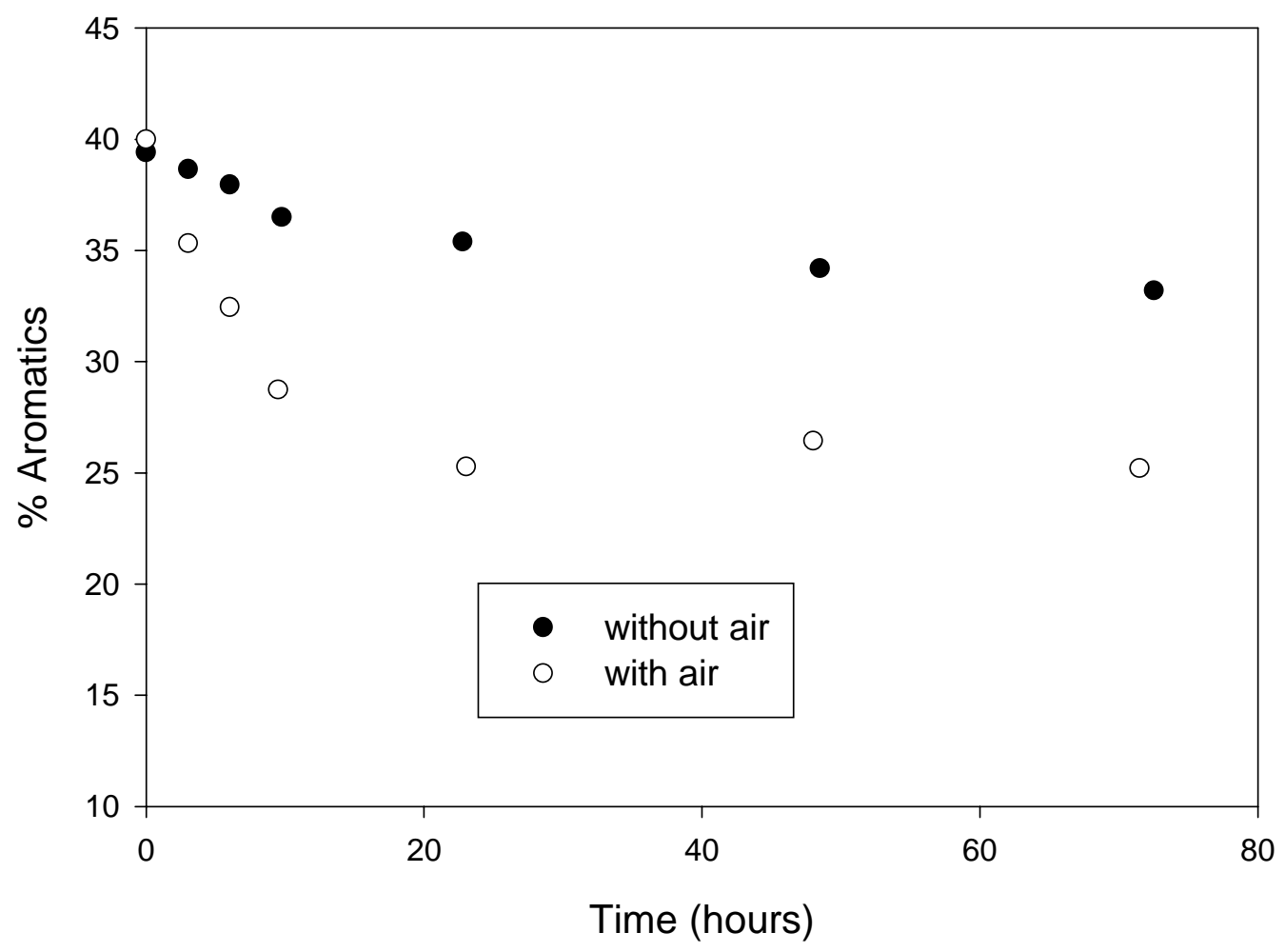

Figure 4.27 - Comparison of Weight Percent Aromatics Stored at $21^{\circ} \mathrm{C}$ With and Without Air (Data Obtained from ELSD) 


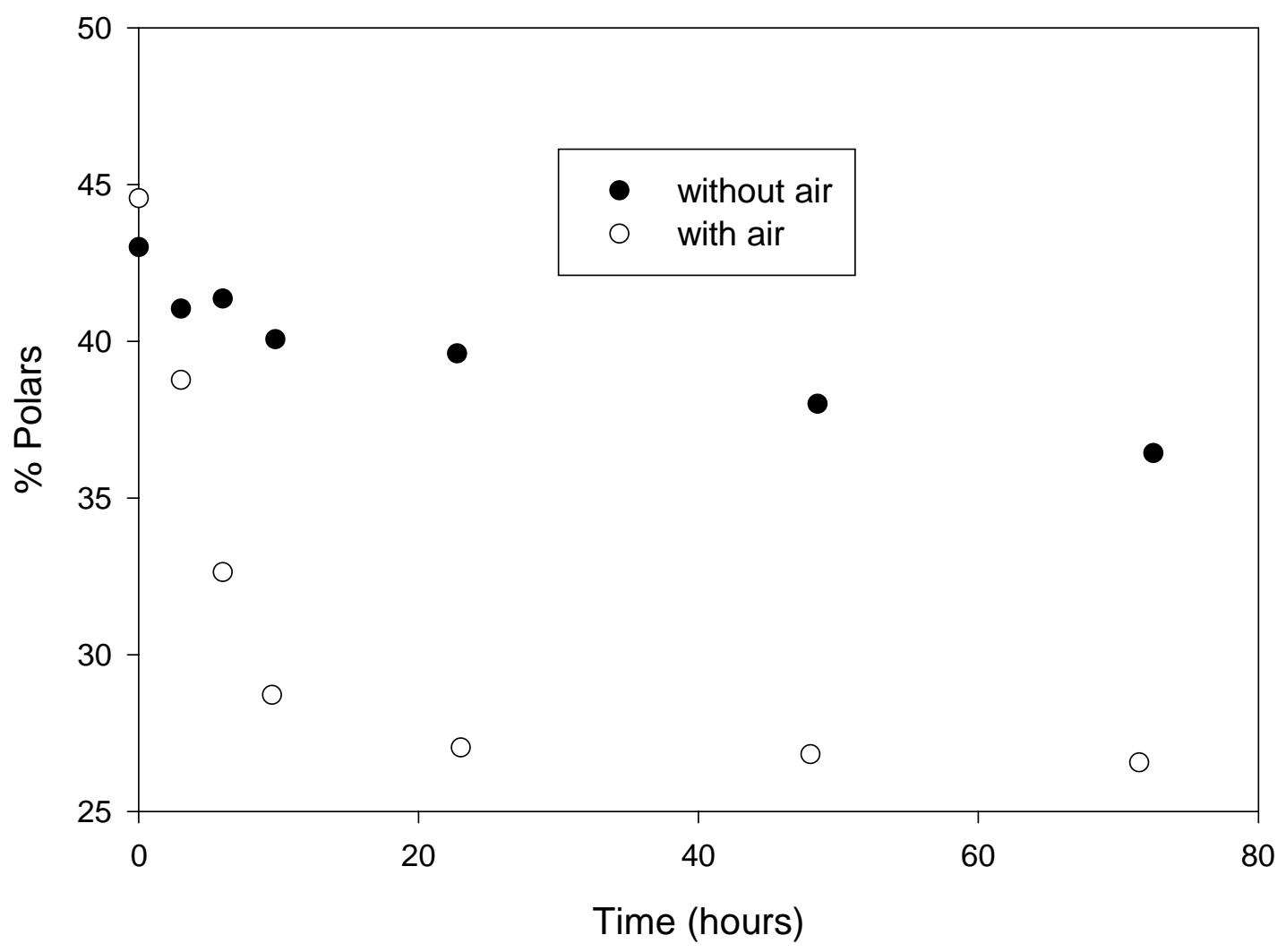

Figure 4.28 - Comparison of Weight Percent Polars Stored at $21^{\circ} \mathrm{C}$ With and Without Air (Data Obtained from ELSD) 


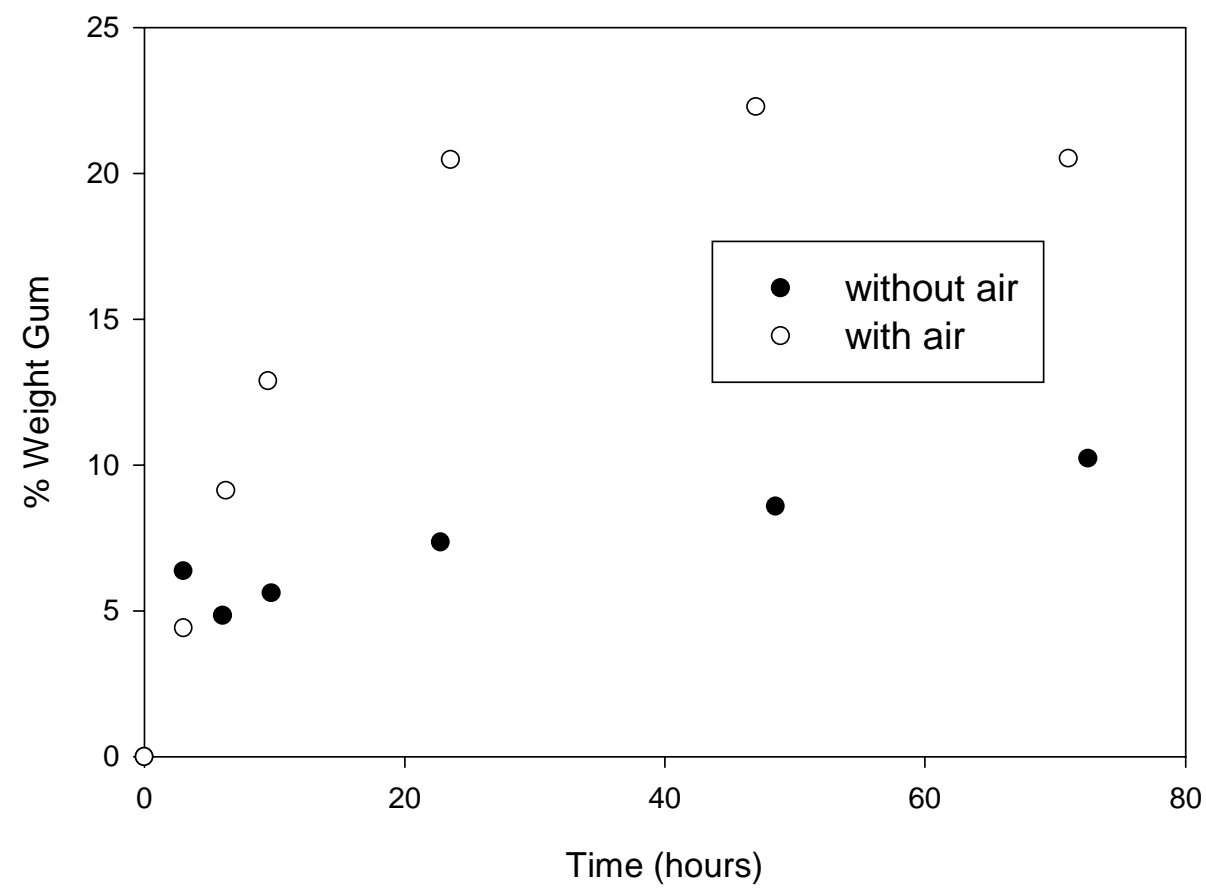

Figure 4.29 - Comparison of Weight Percent Gum Formed Stored at $21^{\circ} \mathrm{C}$ With and Without Air (Data Obtained from ELSD) 
The percent gum formed was also affected by the absence of air. Figure 4.29 shows the comparison of the percent gum formed stored at $21^{\circ} \mathrm{C}$ with and without air. There is a steady but slower increase in gum formation for samples stored with air compared to those stored without air. There was no abrupt increase in gum formation at the early stage of storage in the absence of air. Also, the amount of gum formed is less when stored without air.

The absence of air or the presence of an inert gas when storing coal liquids does not eliminate the formation of gum. Instead, it slows down the rate of gum formation. Brown and Karn [41] observed the same trend when they studied coal liquid samples under a variety of environmental conditions. The rate of viscosity increase is slowest under an inert environment (nitrogen). Their study showed that coal liquid samples had the highest rate of increase in viscosity in the order of oxygen > air > nitrogen gas environment.

Kershaw and Gray [44] also found that the rate of degradation is not zero but slowest when coal liquids are stored in nitrogen gas. Degradation was measured in terms of rate of viscosity increase. The rate of viscosity increase was again found to be greatest on samples stored under oxygen atmosphere.

\subsubsection{The Effect of Oxygen}

The slow increase in gum formation and the slow decrease in aromatic and polar compounds in the absence of air suggests that the oxygen in air plays an important role in gum formation. It has been shown that the presence of oxygen increases the rate of gum formation $[21,22,41,43,44]$. This supports the idea that oxygen in air plays an important role in the degradation reaction. Other degradation 
reactions in the absence of oxygen can also occur as shown in the Figure 4.29 where gum formed at a steady but slow rate. Studies by Brown and Karn [41] and Kershaw and Gray [44] showed that the degradation of coal liquids continues even in the absence of oxygen. Acidic components in the sample mixture may have catalyzed the degradation reaction [65].

The effect of oxygen was not investigated in this study. This would be an interesting topic for future research.

\subsection{Main Reactants in Gum Formation}

It is evident from the literature that the whole picture of fuel stability is very complex. There may be no single explanation for all the phenomena associated with fuel deterioration. However, many investigators have proposed that instability of fuel may be attributed to either oxidation/autoxidation reactions, or polymerization reactions [1]. It seems each type of fuel has its own mechanism for the onset of instability, and there may be several instability reaction mechanisms present in coal liquids. One possible reaction is that between aromatics and polar compounds. The results in Section 4.3 suggest that these two compound classes may be responsible for gum formation. This hypothesis was put to the test in this section. The aromatic compound class will be separated from the polar compound class before storage. 
Coal liquid samples were obtained using the same procedure described in Section 3.3. However, after filtration, the coal liquids were passed through a solidphase extraction tube containing hydroxyl groups similar to those found in the DIOL column from the Hydrocarbon Compound Class Analysis. Figure 4.30 shows the solid phase extraction tube separating the saturated and aromatic groups from the polar groups. The polar compounds were retained in the extraction tube while both the saturates and aromatics passed through the tube. The tube was washed several times with hexane to ensure that neither aromatics nor saturates remained. The polar compounds were eluted from the extraction tube using methylene chloride. Figure 4.31 shows a picture of a fresh sample of coal liquid that has just been separated by the extraction tube. The saturates-and-aromatics appear to be very light yellow-green in color while the polar compounds have a lighter but similar color as the unseparated fresh coal liquid sample. Several sample vials containing saturates-and-aromatics and polar compounds were stored at $2{ }^{\circ} \mathrm{C}, 21^{\circ} \mathrm{C}$, and 32 ${ }^{\circ} \mathrm{C}$. The changes in their composition were monitored using the Hydrocarbon Compound Class Analysis described in Section 3.2. The changes in boiling point distribution were monitored using the Simulated Distillation Analysis described in Section 3.1.

No gum formation was observed from all the polar samples as well as all the aromatic-and-saturate samples stored at $2{ }^{\circ} \mathrm{C}, 21^{\circ} \mathrm{C}$, and $32^{\circ} \mathrm{C}$. All the samples remained clear without any trace of gum deposits to the wall of the sample vials. Moreover, all the samples were allowed to stand for over four months and there were still no signs of gum formation. Other samples showed very light shades of 


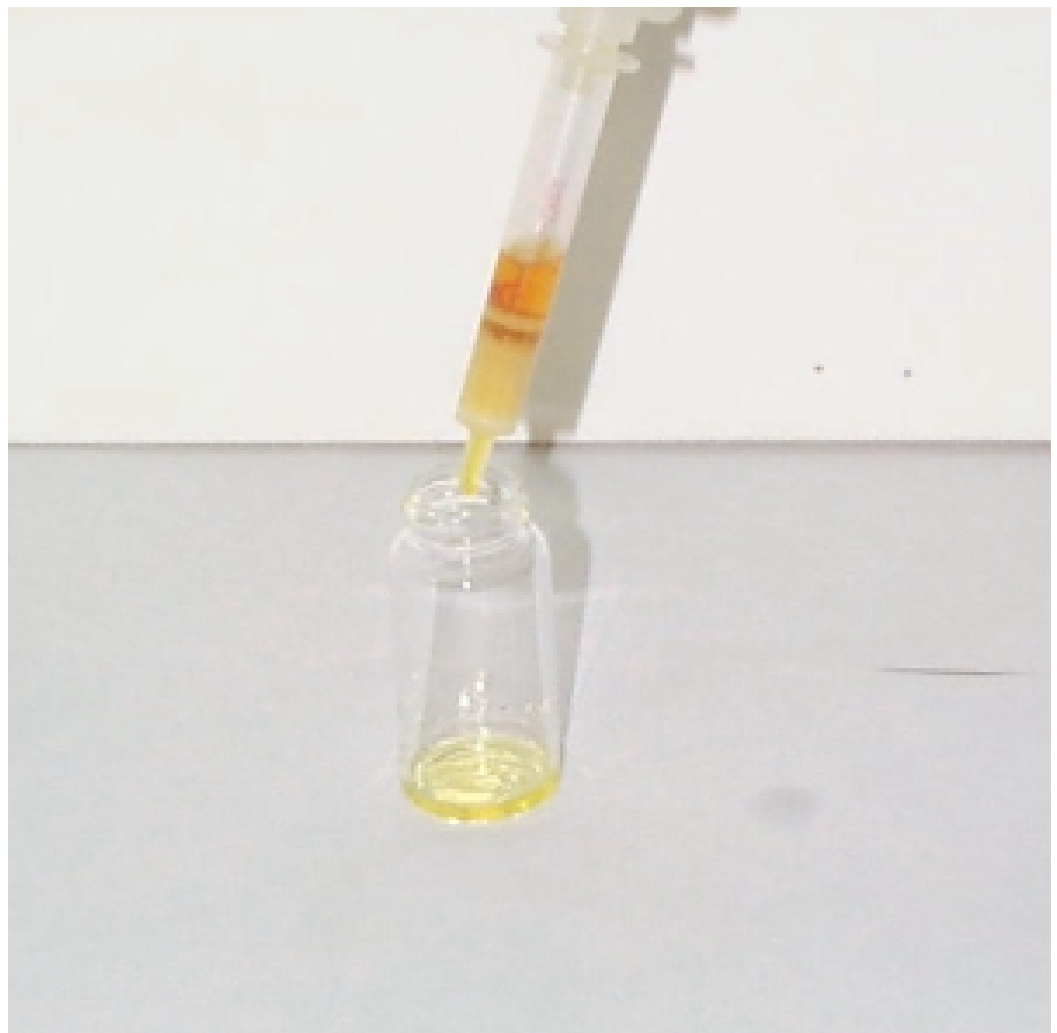

Figure 4.30 - Solid Phase Extraction Tube Separating Polar Compounds from Saturate-and-Aromatic Compounds 


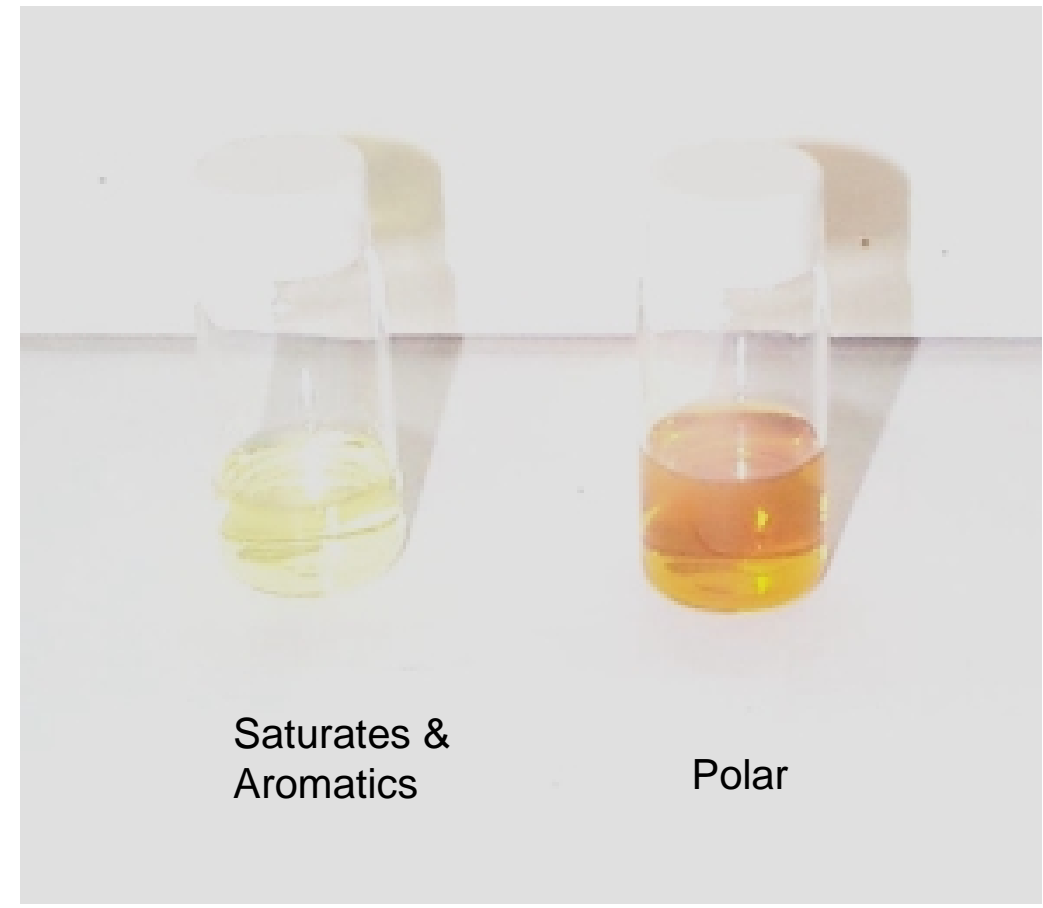

Figure 4.31 - Fresh Saturates-and-Aromatics, and Polar Compounds After Separation by Solid Phase Extraction. 
deposit to the wall of the sample vial after over four months but once the samples were shaken vigorously, the samples became clear.

Figure 4.32 shows the changes in the aromatic-and-saturate groups stored at $2{ }^{\circ} \mathrm{C}, 21^{\circ} \mathrm{C}$ and $32{ }^{\circ} \mathrm{C}$. There were no significant changes in the percent aromatics-and-saturates over a long period of time. Although Figure 4.32 show data up to 72 hours, the samples were also tested after two weeks, one month and two months. The percent aromatics-and-saturates still remained constant with time. There were no apparent reactions between saturates and aromatics during storage.

A similar trend is observed for the polar compounds. Figure 4.33 shows the changes in the polar group stored at $2{ }^{\circ} \mathrm{C}, 21^{\circ} \mathrm{C}$ and $32^{\circ} \mathrm{C}$. The percent polar compounds remained constant with time. The polar samples were also tested after two weeks, one month, and two months and no significant changes were observed during these times.

Correspondingly, there were no changes in the boiling point distribution for all samples stored at $2{ }^{\circ} \mathrm{C}, 21^{\circ} \mathrm{C}$ and $32^{\circ} \mathrm{C}$. The changes in the boiling point range between $315^{\circ} \mathrm{C}$ and $380^{\circ} \mathrm{C}$ are shown in Figures 4.34 and 4.35 , between $380{ }^{\circ} \mathrm{C}$ and $435^{\circ} \mathrm{C}$ in Figures 4.36 and 4.37 , between $435^{\circ} \mathrm{C}$ and $482{ }^{\circ} \mathrm{C}$ in Figures 4.38 and 4.39 , and for boiling point range greater than $482{ }^{\circ} \mathrm{C}$ in Figures 4.40 and 4.41 . 


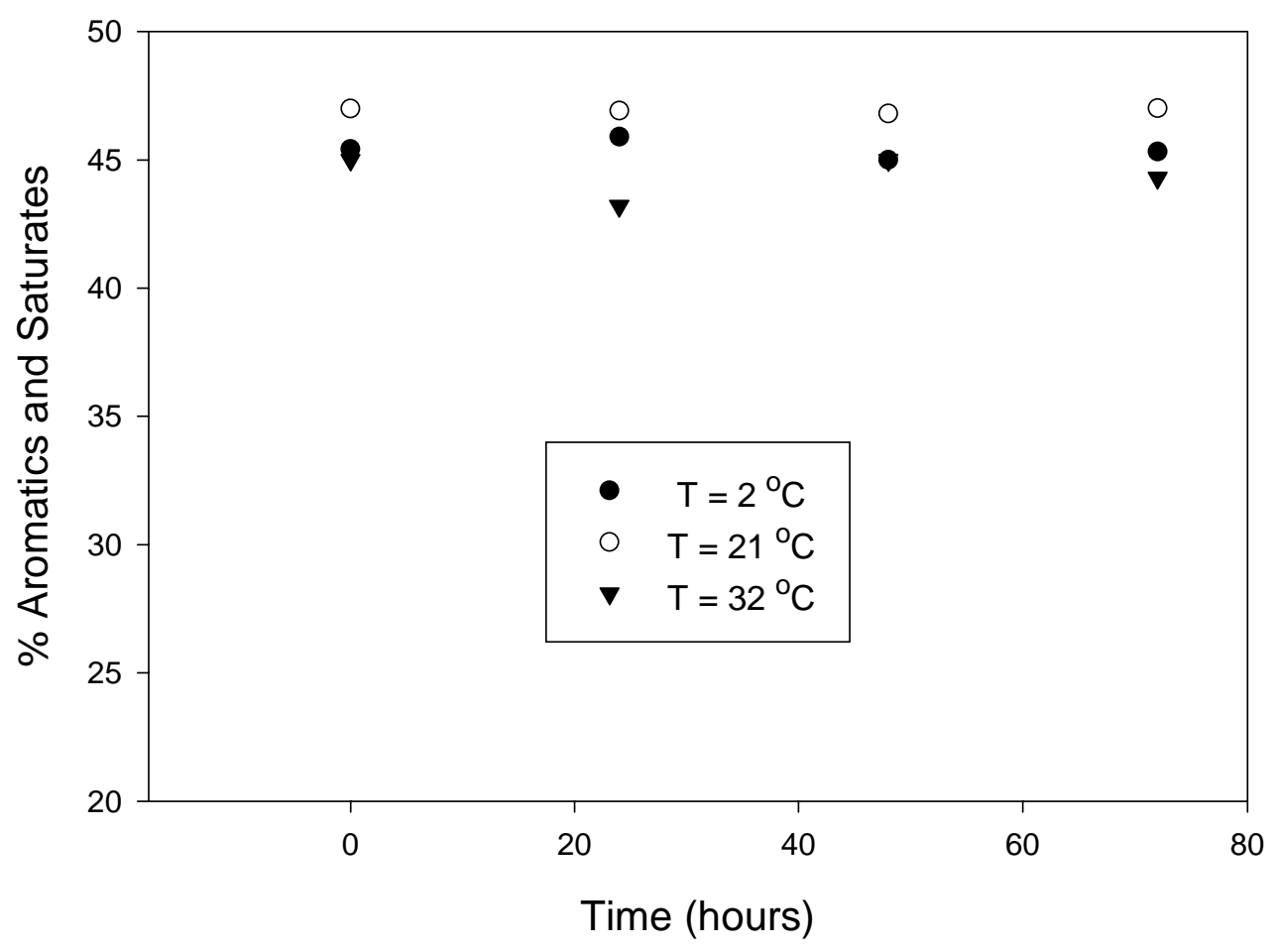

Figure 4.32 - Changes in Saturates-and-Aromatics Stored at 2,21 , and $32{ }^{\circ} \mathrm{C}$ 


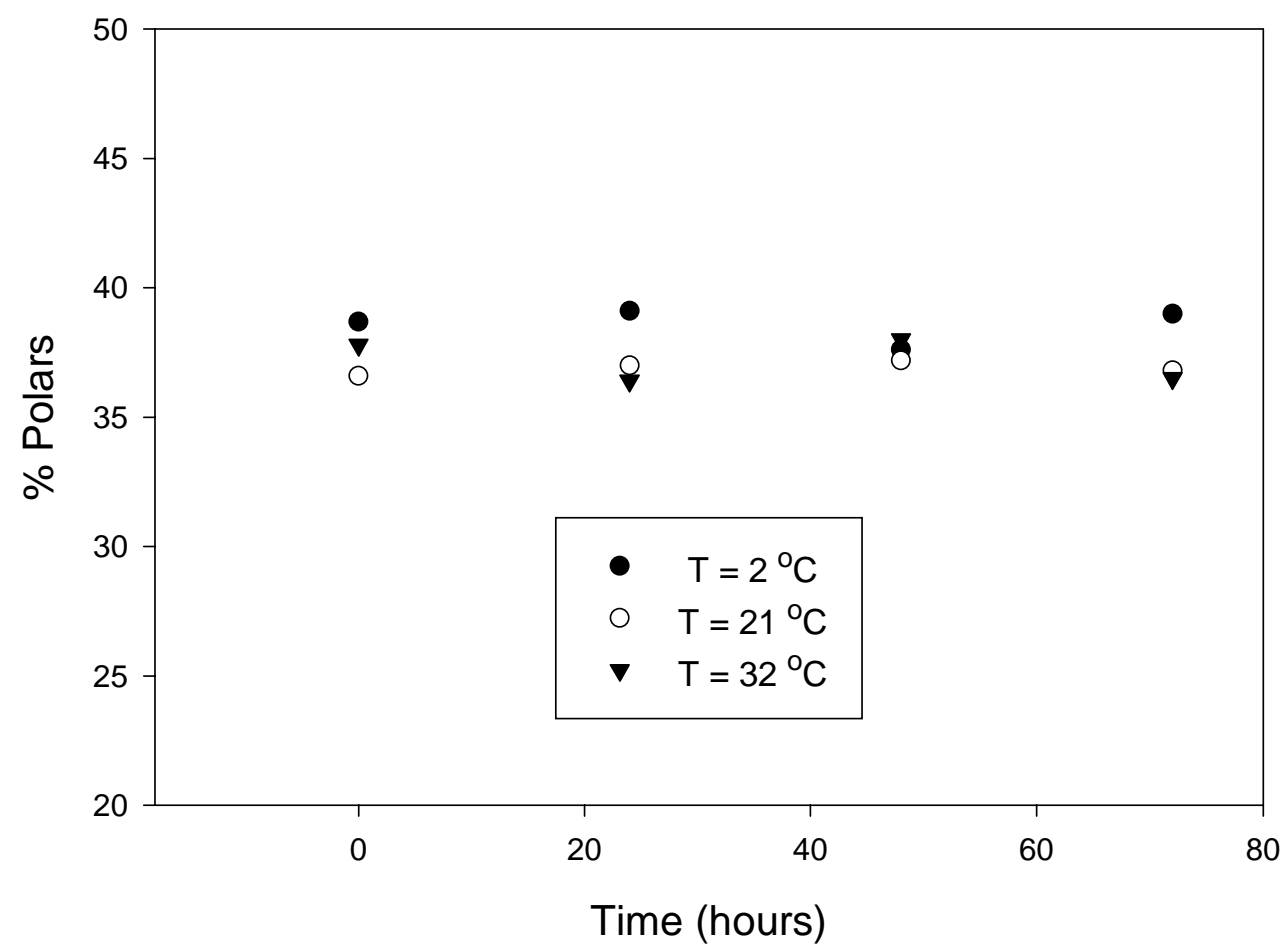

Figure 4.33 - Changes in Polars Stored at 2, 21, and $32{ }^{\circ} \mathrm{C}$ 


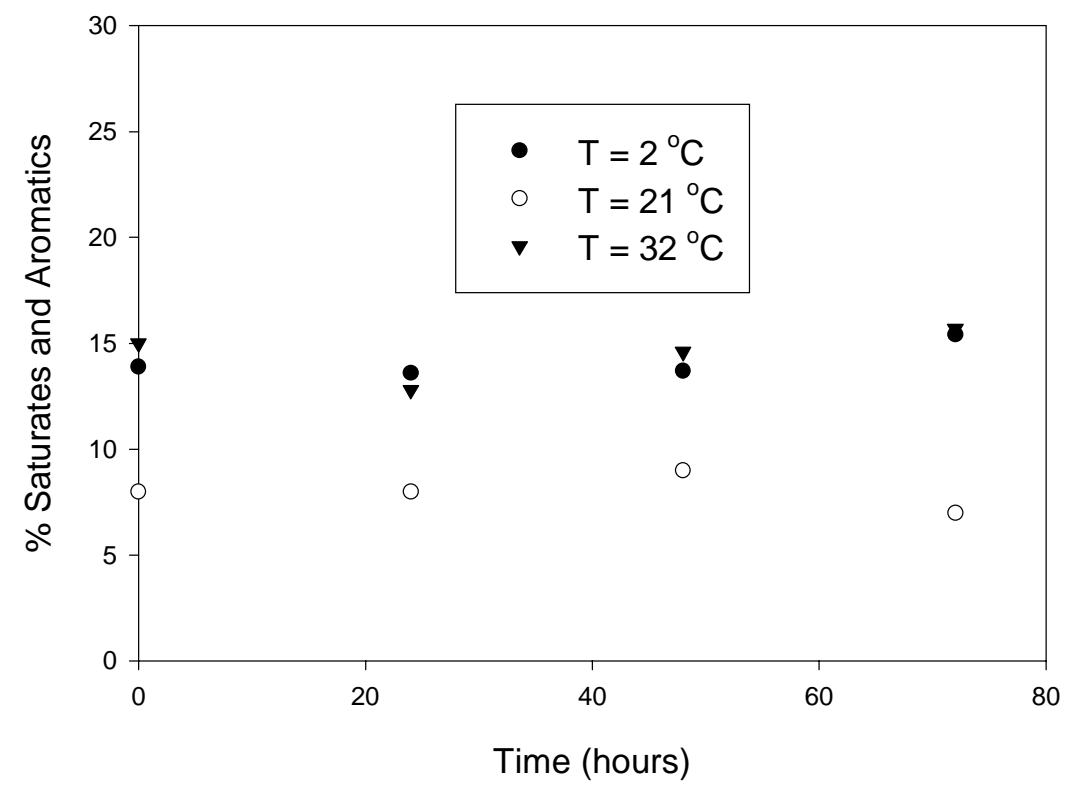

Figure 4.34 - Changes in Boiling Point Distribution in $315^{\circ} \mathrm{C}>\mathrm{BP}>380^{\circ} \mathrm{C}$ for Saturates-and-Aromatics

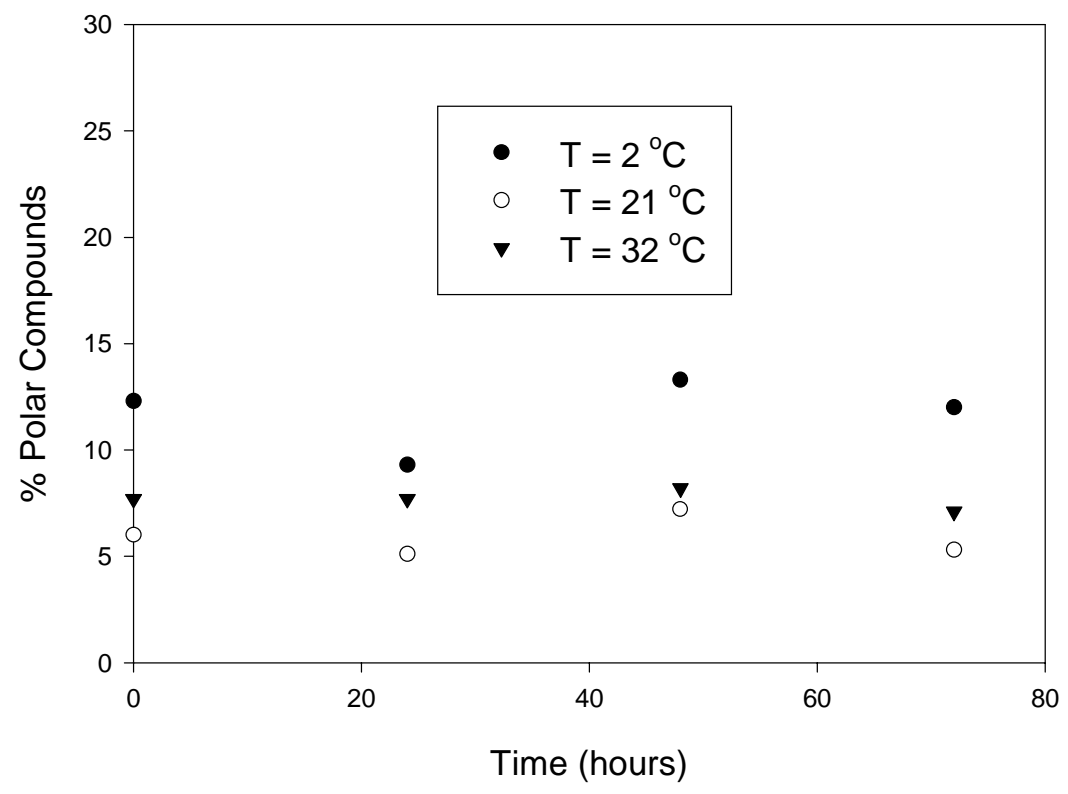

Figure 4.35 - Changes in Boiling Point Distribution in $315^{\circ} \mathrm{C}>\mathrm{BP}>380^{\circ} \mathrm{C}$ for Polar Compounds 


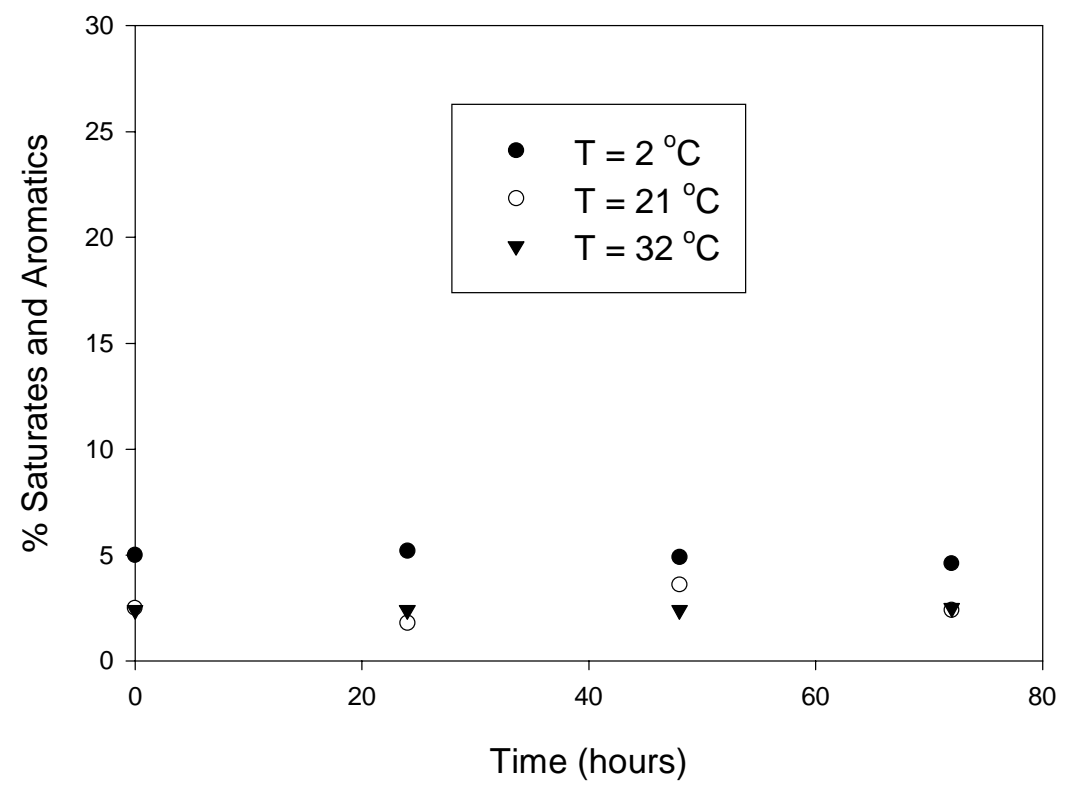

Figure 4.36 - Changes in Boiling Point Distribution in $380{ }^{\circ} \mathrm{C}>\mathrm{BP}>435{ }^{\circ} \mathrm{C}$ for Saturates-and-Aromatics

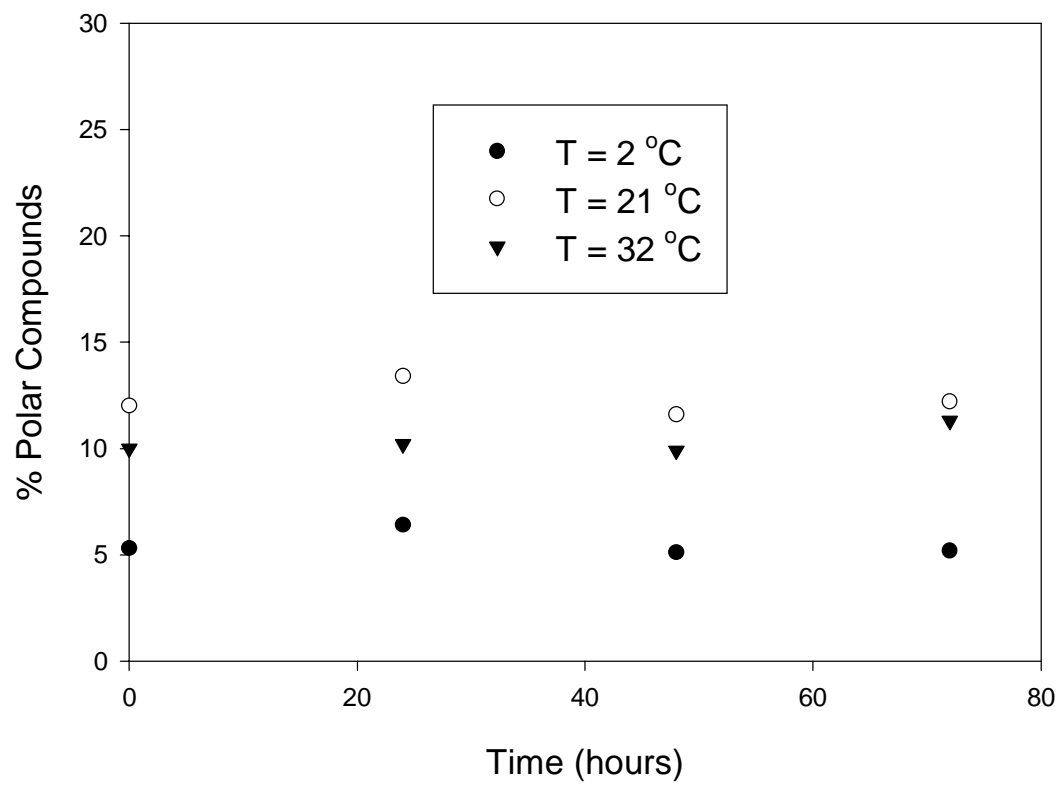

Figure 4.37 - Changes in Boiling Point Distribution in $380^{\circ} \mathrm{C}>\mathrm{BP}>435^{\circ} \mathrm{C}$ for Polar Compounds 


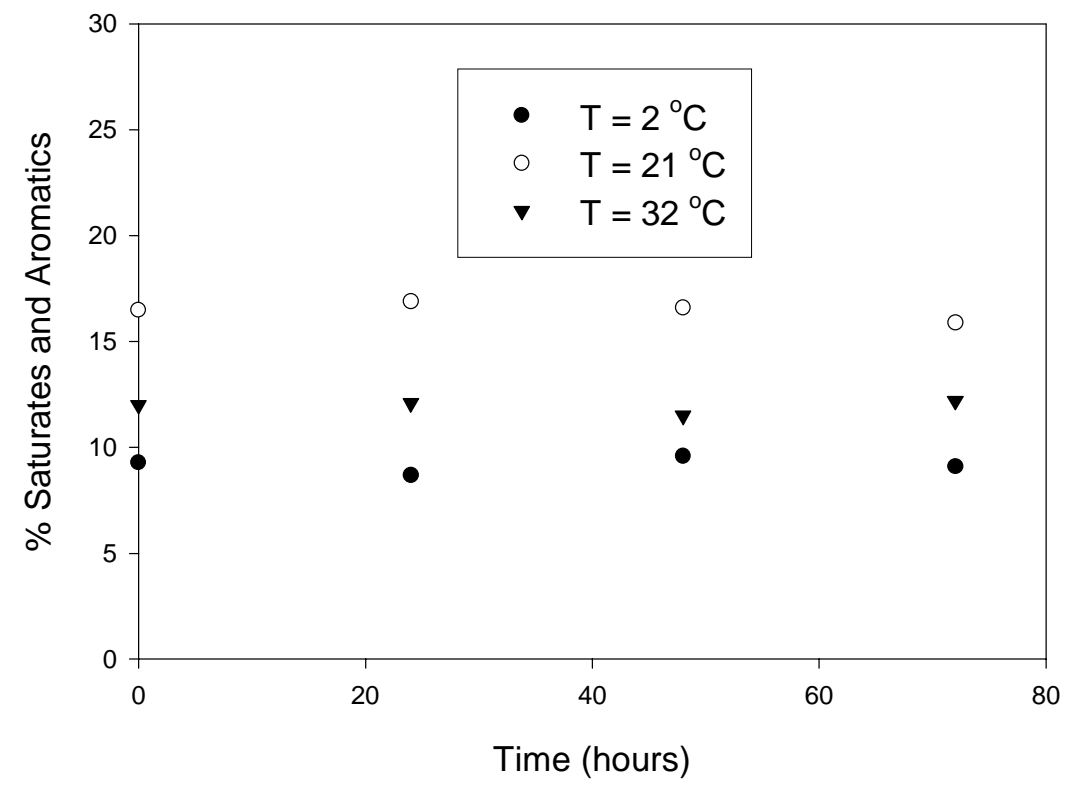

Figure 4.38 - Changes in Boiling Point Distribution in $435^{\circ} \mathrm{C}>\mathrm{BP}>482{ }^{\circ} \mathrm{C}$ for Saturates-and-Aromatics

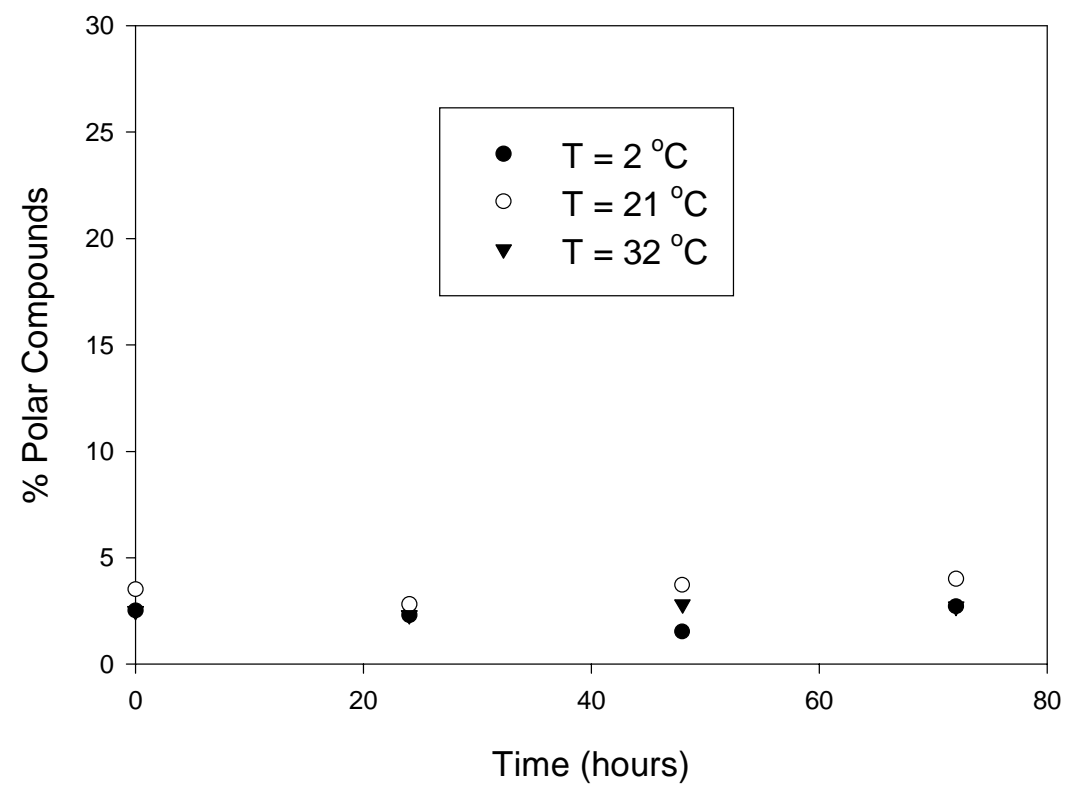

Figure 4.39 - Changes in Boiling Point Distribution in $435^{\circ} \mathrm{C}>\mathrm{BP}>482{ }^{\circ} \mathrm{C}$ for Polar Compounds 


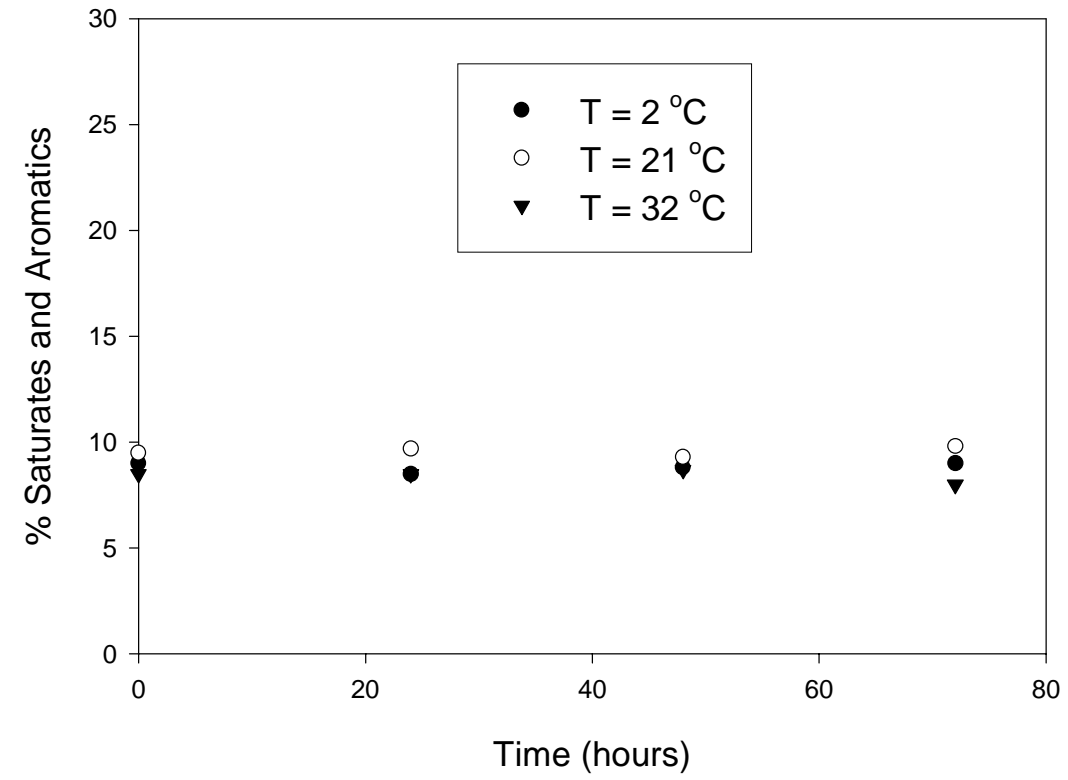

Figure 4.40 - Changes in Boiling Point Distribution in BP $>482{ }^{\circ} \mathrm{C}$ for Saturates-and-Aromatics

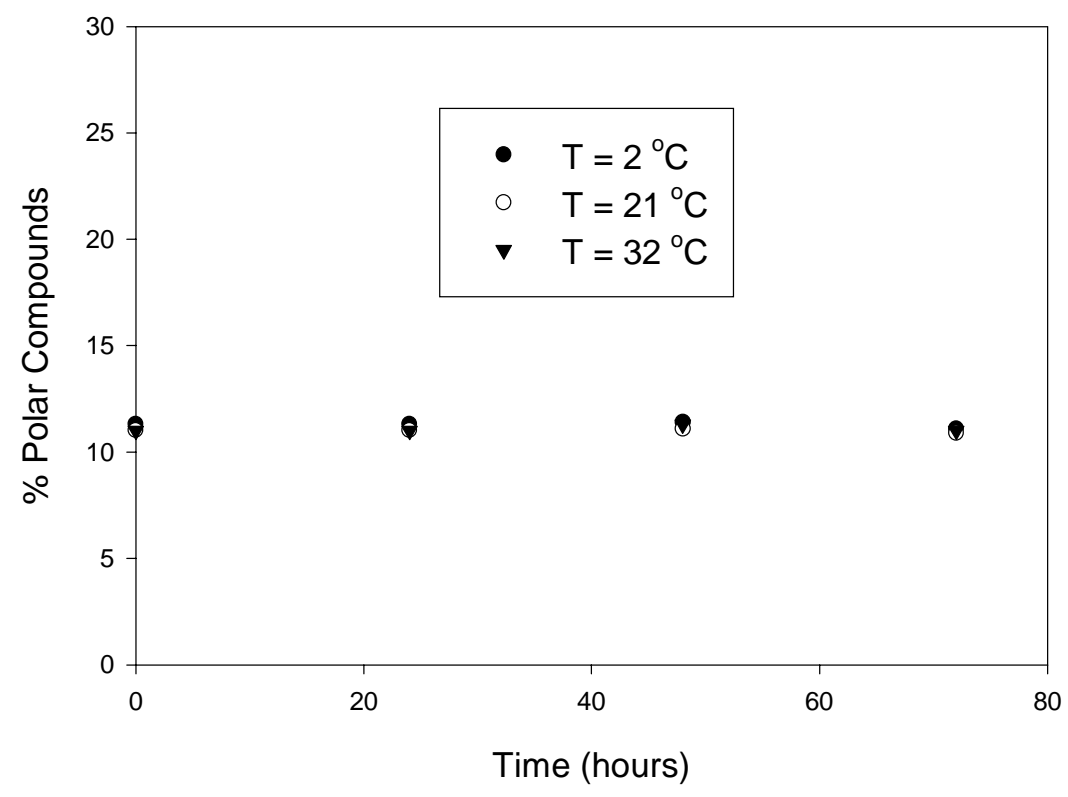

Figure 4.41 - Changes in Boiling Point Distribution in BP $>482{ }^{\circ} \mathrm{C}$ for Polar Compounds 
Since there was no observable gum formation after the separation of polar compounds from the saturates-and-aromatics, the polar compounds were mixed once again with the saturates-and-aromatics. Four days after mixing the two samples, the mixture started forming gum. More gum was produced after one week. Unlike before, the gums produced here did not adhere to the wall of the glass vial; instead, the gum settled to the bottom of the sample vial.

The delay of four days before the gum started to appear may be due to the fact that the polar compounds were in methylene chloride solution. This is different from the original storage conditions wherein all coal liquids were in hexane solution. This time, all the compound classes were in a mixture of hexane and methylene chloride solution. Following the analysis discussed in Section 4.2, the saturation limit may have changed because of the difference in solubility of the gum to the methylene chloride in the mixture. However, once this saturation limit is reached, the gum started to precipitate out from the mixture. Thus, based on these results, it can be concluded that the main reactants in gum formation are the aromatic and the polar compound classes. The difference in solubility of gum to methylene chloride compared to that of hexane caused the delay in the formation of gum. This suggests that the kinetics of gum formation is a function of the solvent. 


\subsection{Polar Compounds}

To determine the primary compounds present in the polar compound class fraction, the polar compounds were analyzed using Gas Chromatography/Mass Spectrometry (GC/MS) and Fourier Transform Infrared Spectroscopy (FTIR). The polar compounds were isolated from fresh coal liquid samples by solid phase extraction as described in Section 4.6. The polar compounds were eluted using methylene chloride. The resulting solution was concentrated by evaporation at room temperature.

\subsubsection{GC/MS Results}

The GC/MS system used was a Varian Saturn GC/MS 3 with an MS library search program. The GC is equipped with a 1077 split/splitless capillary injector, and a DB5-MS narrowbore, capillary column $(30 \mathrm{~m} \times 0.25 \mathrm{~mm}$ i.d., $0.25 \mu \mathrm{m}$ film thickness, J \& W Scientific). Helium was used as the carrier gas. The GC oven started at $60{ }^{\circ} \mathrm{C}$ and was then temperature programmed at $5{ }^{\circ} \mathrm{C} / \mathrm{min}$ to a final temperature of $315^{\circ} \mathrm{C}$ and held there for 9.5 minutes.

The polar compound mixture was injected into the GC/MS for identification. The experiments were carried out using the Electron Impact (EI) mode where the molecules are bombarded in the vapor phase with a high energy electron beam. The result of electron impact is recorded as a spectrum based on mass/charge $(\mathrm{m} / \mathrm{z})$. Since it is already known that the mixture contains only one type of compound class (polars), the MS library was adequate for identification. Before matching the sample spectrum with the stored spectra in the library, background 
subtraction was done to remove unwanted chemical background so that an accurate library search could be done. Background subtraction enhances mass spectral data to ensure that the final spectrum shows only those masses that result from the analyte. These unwanted peaks may be due to column bleeding.

Figure 4.42 shows the total ion chromatogram resulting from the GC/MS analysis of the polar compounds. The largest peak eluted at 12.50 minutes is identified as the methylene chloride solvent. Prominent peaks are found at masses corresponding to phenol and its homologues (alkyl derivatives). These prominent peaks are at $\mathrm{m} / \mathrm{z} 94,108,122$ and 135 . The rest of the peaks gave weak signals and several ambiguities in their mass spectra.

The sample mass spectra of the prominent peaks, 94, 108 (two isomers), 122 (three isomers) and 135, and their respective possible matches from the MS Library are shown in Figures 4.43 to 4.49 . Excellent matches were obtained when the mass spectra were compared to the ones present in the library. A list of polar compounds identified by the GC/MS is shown in Table 4.9. The polar compound class isolated from the coal liquid consists mainly of phenol and its homologues. This result is consistent with the literature. Hara et al. [43] also identified the presence of phenols in their study of the aging characteristics of SRC I and SRC II middle distillates. Brown and Karn [41] found an appreciable concentration of phenols in coal liquids. Viscometric, spectrometric, and ultimate analysis were employed to monitor the storage properties of coal derived liquids. 


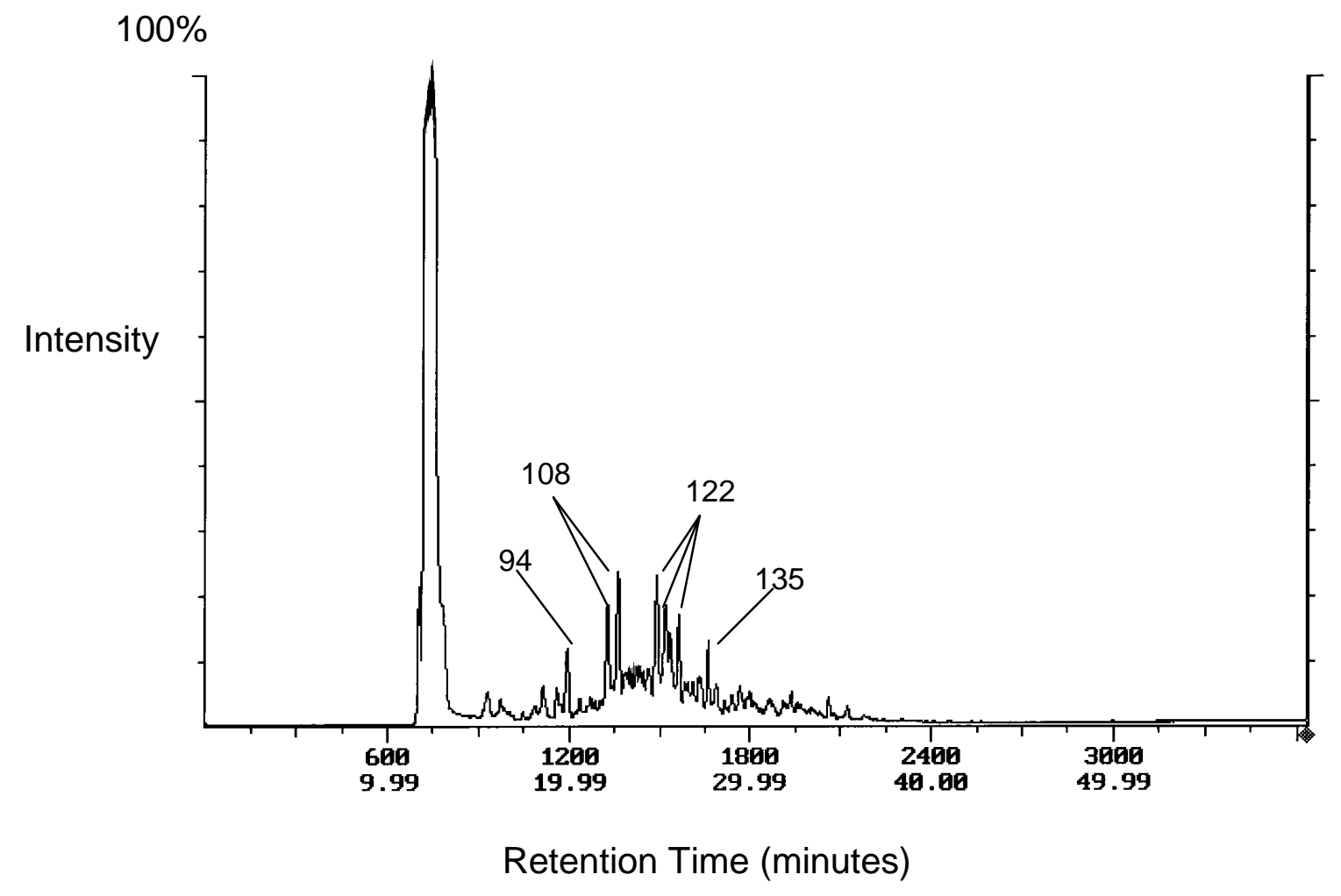

Figure 4.42 - GC/MS Total lon Chromatogram of Polar Compounds (Masses of Prominent Peaks are Shown) 


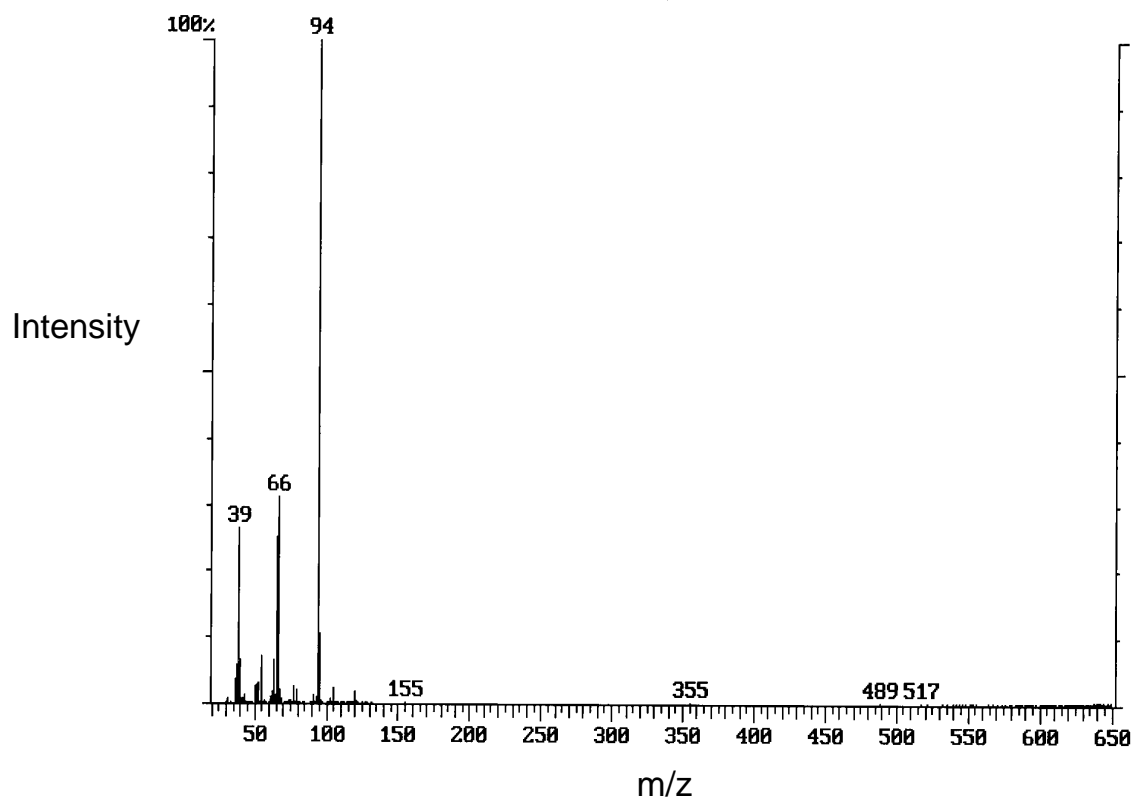

(a)

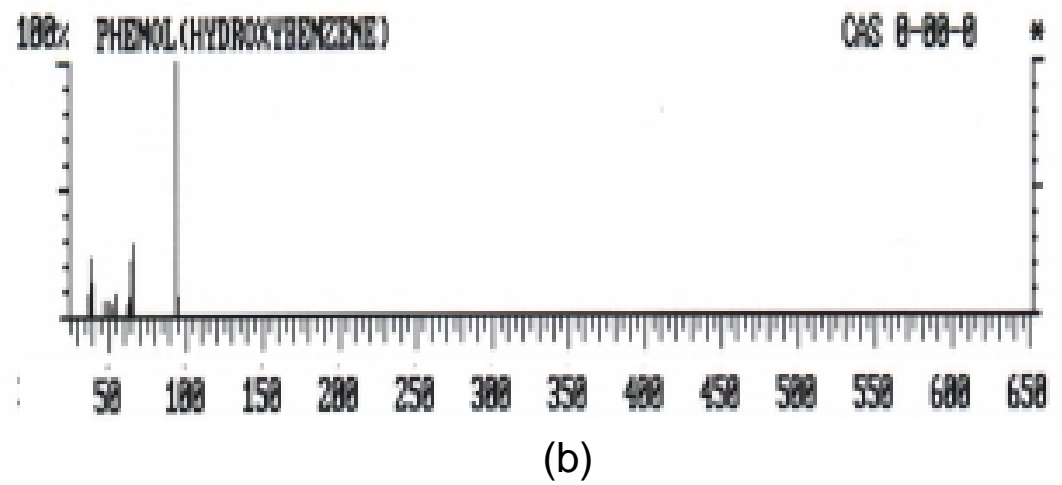

Figure 4.43 - (a) Mass Spectrum of Sample

(b) Possible Match of Phenol from MS Library 


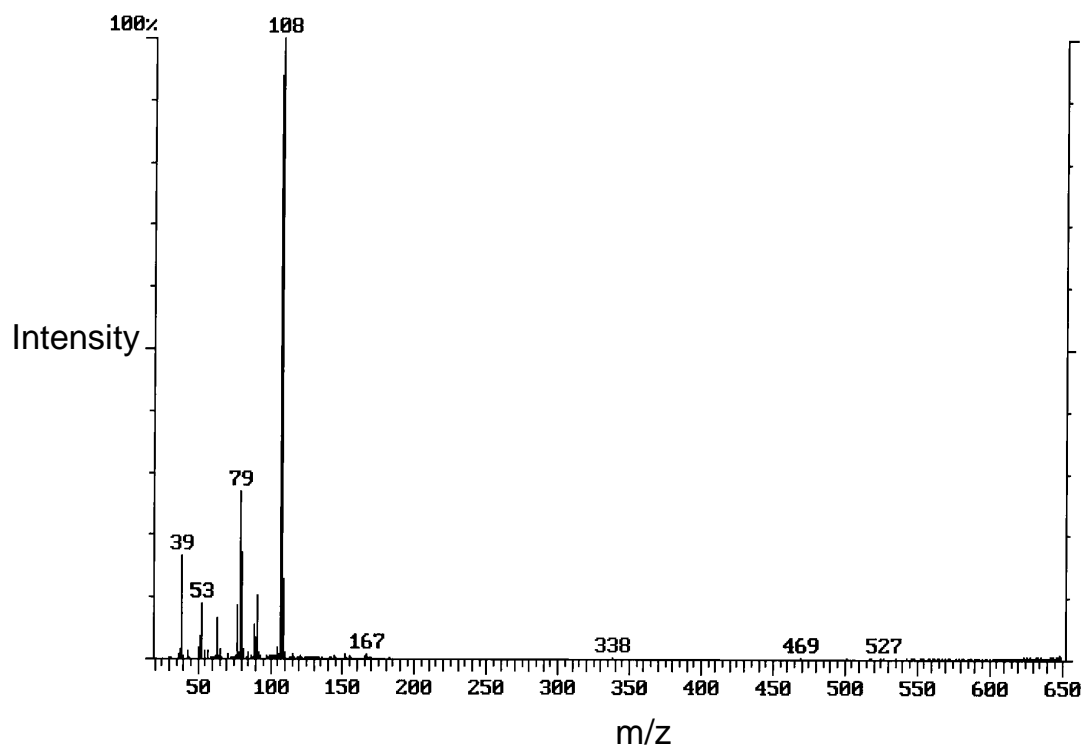

(a)

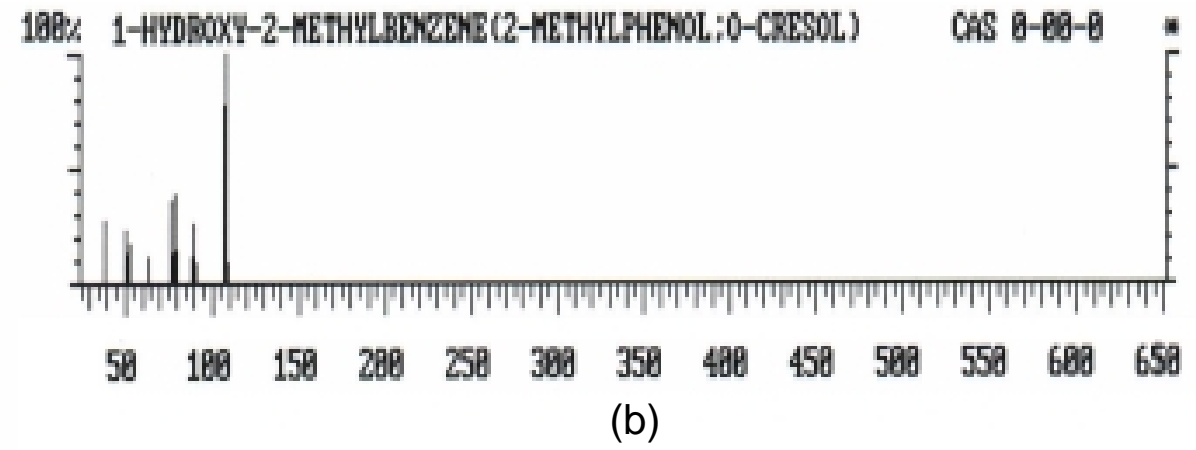

Figure 4.44 - (a) Mass Spectrum of Sample, (b) Possible Match of 2 - methyl phenol from MS Library 


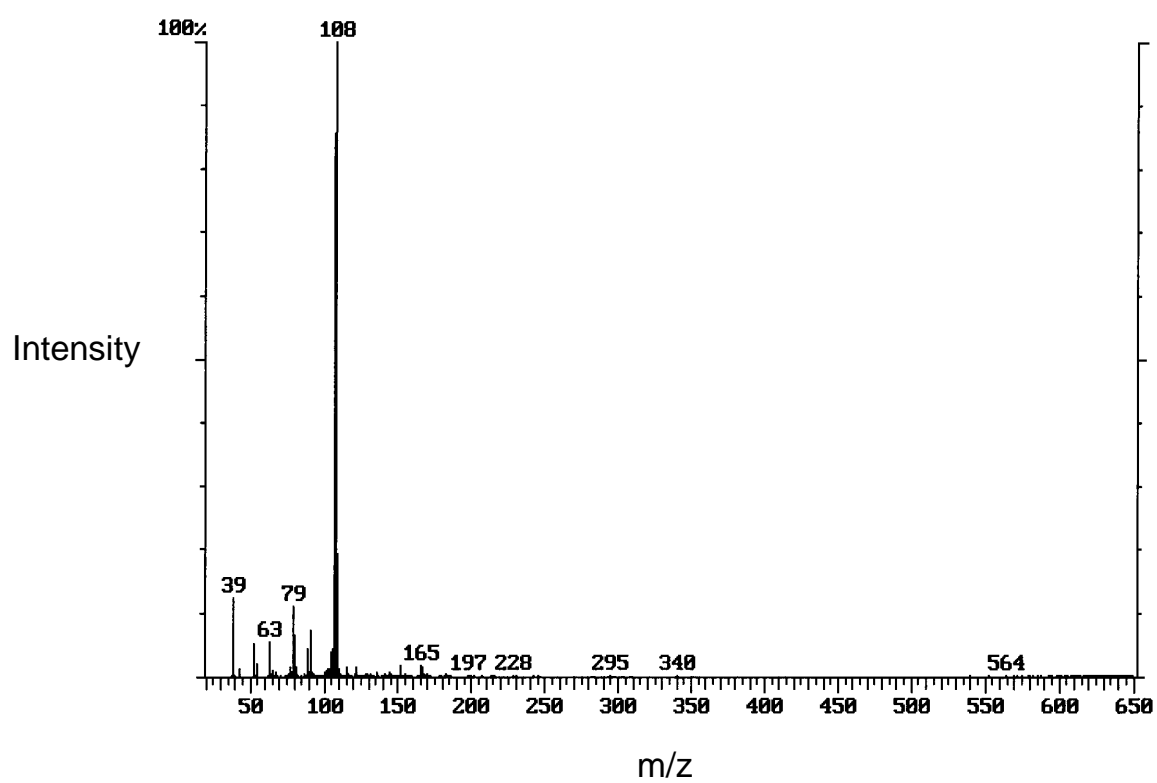

(a)

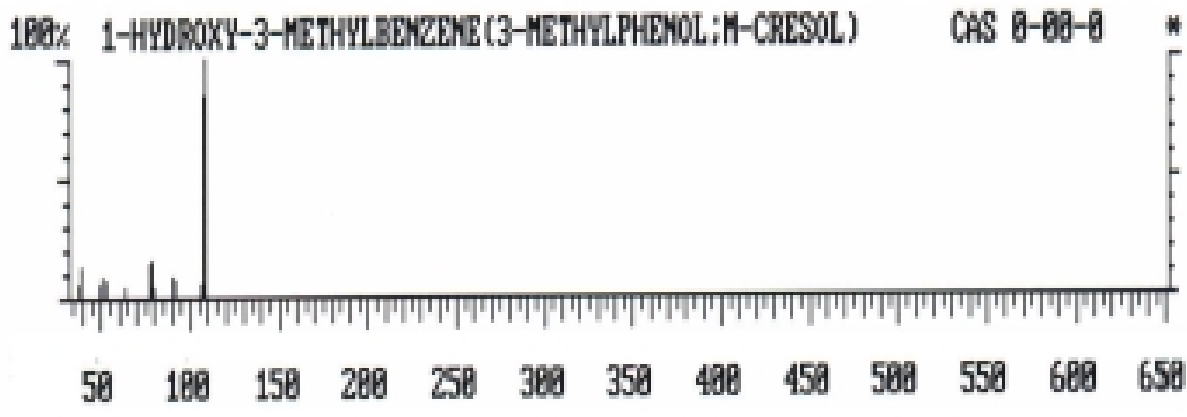

(b)

Figure 4.45 - (a) Mass Spectrum of Sample, (b) Possible Match of 3 - methyl phenol from MS Library 


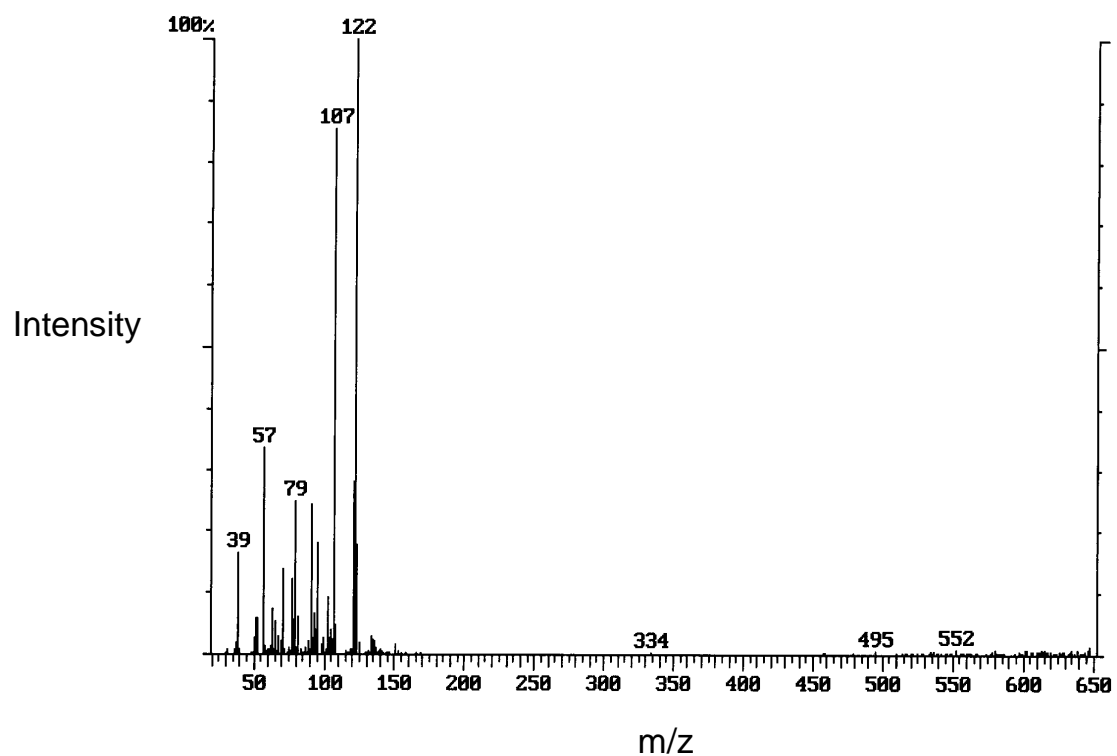

(a)

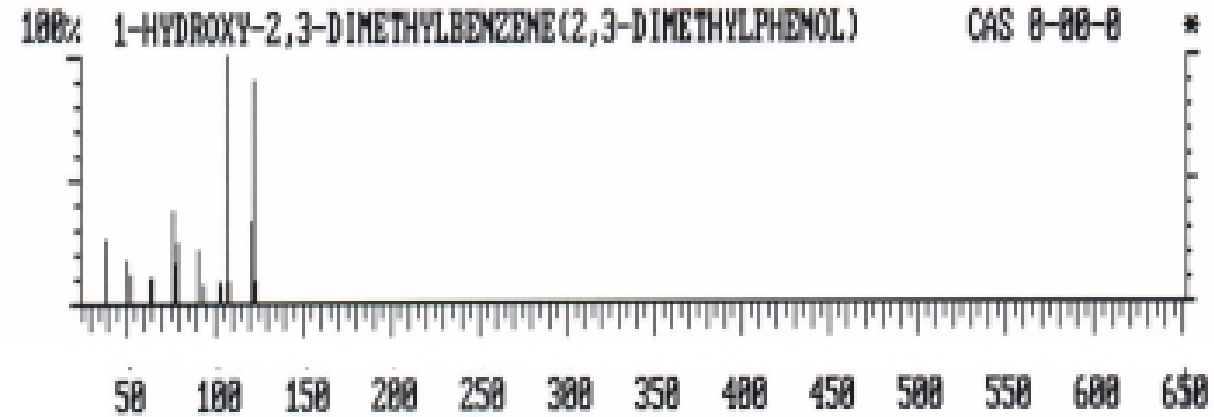

(b)

Figure 4.46 - (a) Mass Spectrum of Sample, (b) Possible Match of 2, 3 - dimethyl phenol from MS Library 


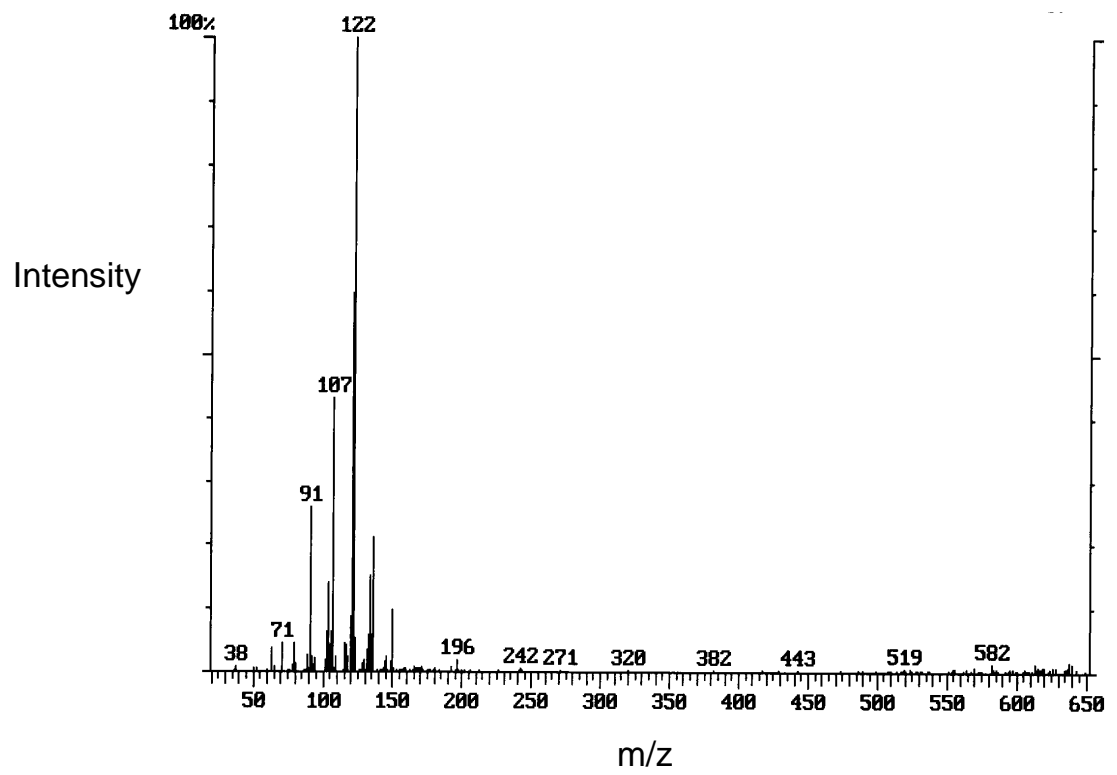

(a)

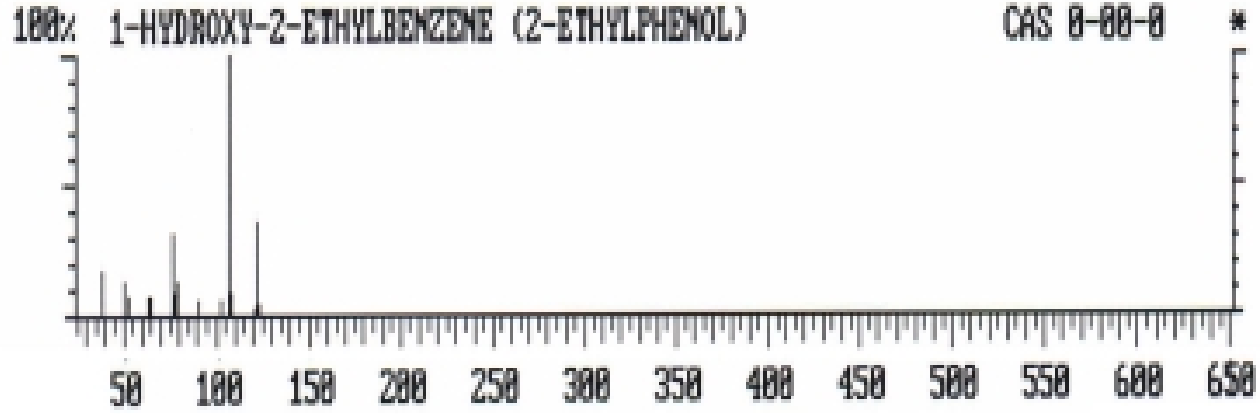

(b)

Figure 4.47 - (a)Mass Spectrum of Sample, (b) Possible Match for 2 - ethyl phenol from MS Library 


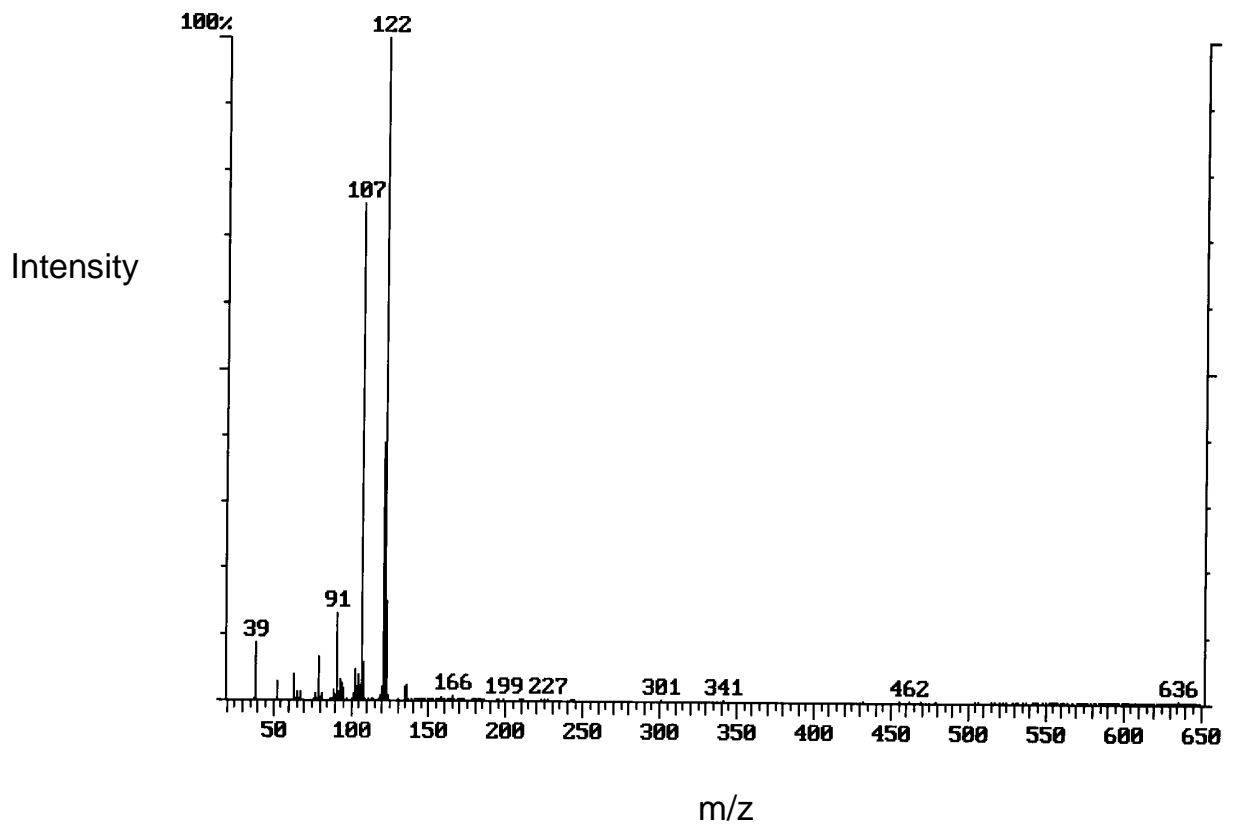

(a)

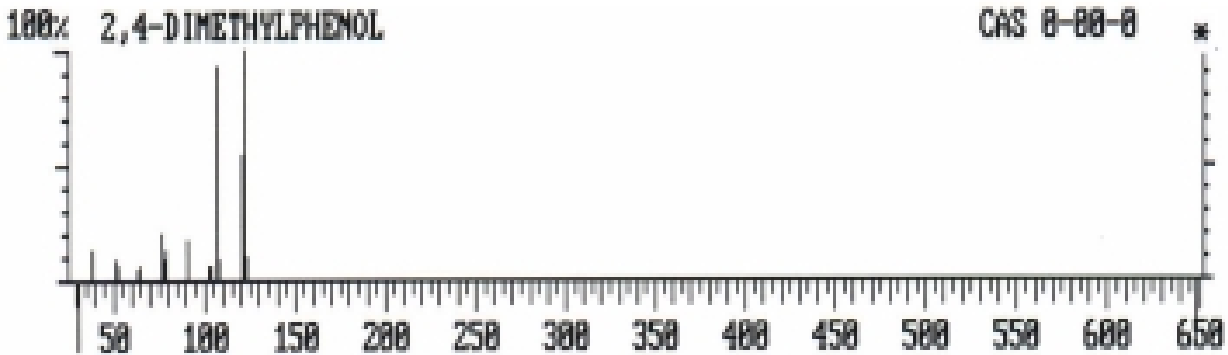

(b)

Figure 4.48 - (a)Mass Spectrum of Sample, (b) Possible Match of 2, 4 - dimethyl phenol from MS Library 


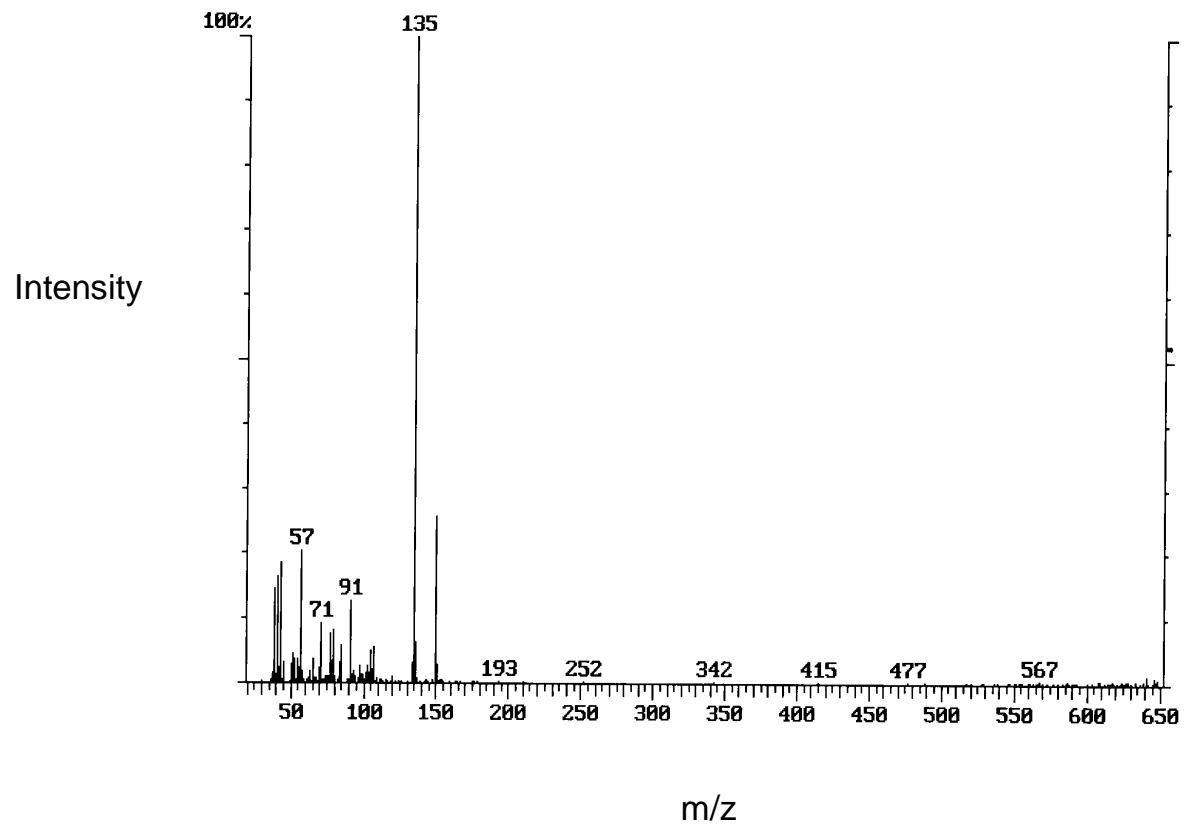

(a)

180\% 3-ETHYL-5- HETHYPHENOL

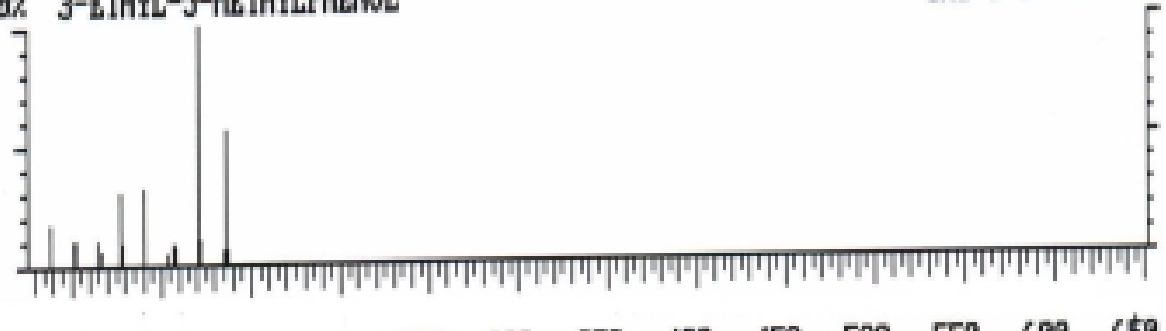

$\begin{array}{lllllllllllll}58 & 180 & 158 & 289 & 258 & 360 & 350 & 498 & 458 & 580 & 558 & 680 & 658\end{array}$

(b)

Figure 4.49 - (a)Mass Spectrum of Sample, (b) Possible Match of 3 - ethyl - 5 - methyl phenol from MS Library 
Table 4.9. List of Polar Compounds Identified by GC/MS

\begin{tabular}{|c|c|c|c|c|}
\hline $\begin{array}{c}\text { Polar } \\
\text { Compound }\end{array}$ & Formula & $\mathrm{m} / \mathrm{z}$ & $\begin{array}{c}\text { Molecular } \\
\text { Weight }\end{array}$ & $\begin{array}{c}\text { Fit } \\
(\%)\end{array}$ \\
\hline Phenol & $\mathrm{C}_{6} \mathrm{H}_{6} \mathrm{O}$ & 94 & 94 & 97.6 \\
\hline $\begin{array}{c}\text { 2-methyl } \\
\text { phenol }\end{array}$ & $\mathrm{C}_{7} \mathrm{H}_{8} \mathrm{O}$ & 108 & 108 & 98.8 \\
\hline $\begin{array}{c}\text { 3-methyl } \\
\text { phenol }\end{array}$ & $\mathrm{C}_{7} \mathrm{H}_{8} \mathrm{O}$ & 108 & 108 & 92.1 \\
\hline $\begin{array}{c}\text { 2, 3-dimethyl } \\
\text { phenol }\end{array}$ & $\mathrm{C}_{8} \mathrm{H}_{10} \mathrm{O}$ & 122 & 122 & 98.8 \\
\hline $\begin{array}{c}\text { 2-ethyl } \\
\text { phenol }\end{array}$ & $\mathrm{C}_{8} \mathrm{H}_{10} \mathrm{O}$ & 122 & 122 & 97.6 \\
\hline $\begin{array}{c}\text { 2,4-dimethyl } \\
\text { phenol }\end{array}$ & $\mathrm{C}_{8} \mathrm{H}_{10} \mathrm{O}$ & 122 & 122 & 97.7 \\
\hline $\begin{array}{c}\text { 3-ethyl-5- } \\
\text { methylphenol }\end{array}$ & $\mathrm{C}_{9} \mathrm{H}_{12} \mathrm{O}$ & 135 & 136 & 93.4 \\
\hline
\end{tabular}

Ueda et al. [67] characterized the chemical composition of coal-derived liquids using field ionization MS. The sample separation was carried out using HPLC. They found the presence of phenols, naphthylphenols, indanols, phenanthrols, dihydrophenanthrols, tetrahydrophenanthrols and octahydrophenantrols in the coal liquid sample.

White et al. [68] found that the concentration of phenols decreased after aging. Characterization of the aging product was done using a low-voltage-highresolution mass spectrometry. They suggested that removal of phenols by further hydrogenation of coal-derived liquids to convert the phenolic oxygen to water may reduce the aging process. 


\subsubsection{FTIR Results}

Figure 4.50 shows the infrared spectrum of the polar compound class. The $\mathrm{O}-\mathrm{H}$ stretching vibrations gave a very strong and very broad absorption peak in the $3200-3600 \mathrm{~cm}^{-1}$ wave number region that is a characteristic vibration for alcohols and phenols [69]. A medium absorption peak at $2853-2962 \mathrm{~cm}^{-1}$ is observed for the $\mathrm{C}-\mathrm{H}$ stretching vibrations that is consistent with the presence of $-\mathrm{CH}_{3}$, and $-\mathrm{CH}_{2}-$ groups. This result confirmed the presence of phenol and its alkyl derivatives that were identified by GC/MS in Section 4.7.1. 


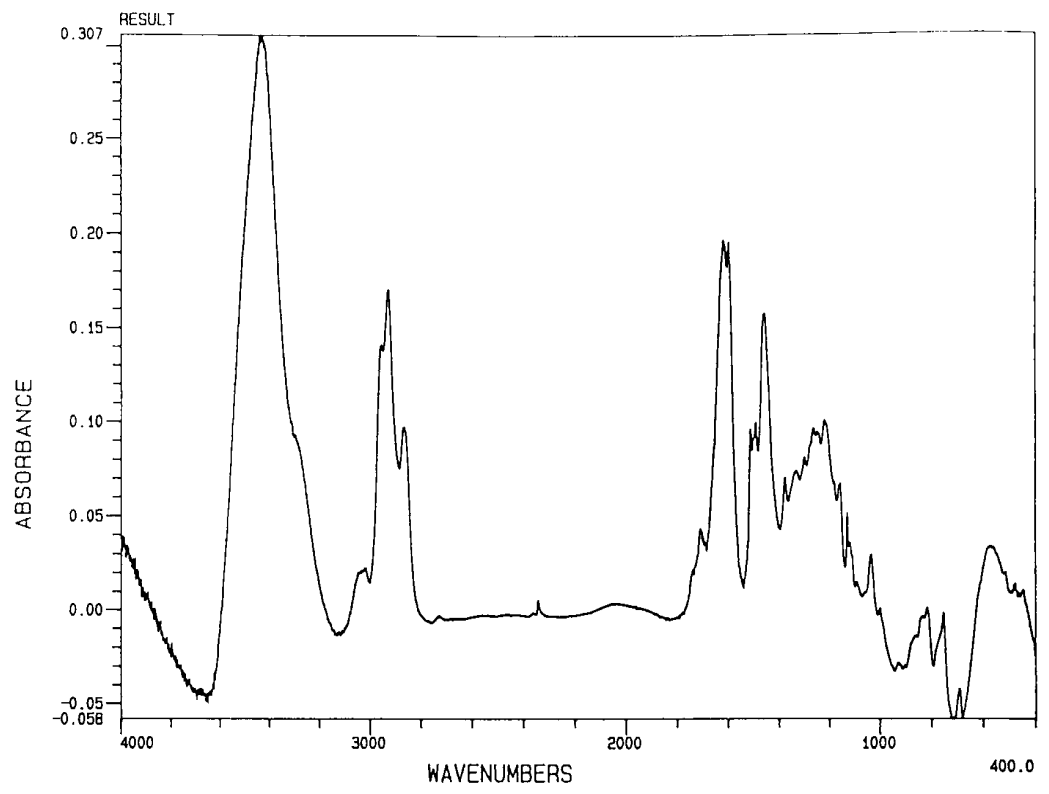

Figure 4.50 - Infrared Spectrum of the Polar Compound Class 


\subsection{Reaction Kinetics}

\subsubsection{Overview of the Degradation Reaction}

At a recent symposium on storage stability of fuels, Dorbon [64] stated, "The degradation mechanisms have not yet been clearly identified despite many interesting investigations already conducted." Active nitrogen compounds are known to induce instability by promoting the formation of sediment. Frankenfeld and co-workers [7] from their study of storage stability of synfuels from oil shale, investigated the structure of sediments obtained from sedimentation in model petroleum-derived fuel by using pure nitrogen compounds. They analyzed the sediment by infrared and mass spectrometric methods and reported that the sediment consisted mainly of partly-oxidized trimers and tetramers of the parent nitrogen compound. Brinkman et al. [6] found that the effects of nitrogen compounds on gum formation depend upon their structure. The position of the alkyl groups on the heterocyclic ring plays an important role. Nonbasic nitrogen heterocyclics with alkyl groups on the ring carbon adjacent to nitrogen were highly reactive. They also concluded that interactions between nitrogen compounds can have significant effects on sediment formation.

The effect of sulfur-containing compounds on the storage stability of Jet A fuels was investigated by Heneman [34]. It was found that thiols and thiophene derivatives added to jet fuels increased the rate of gum formation compared to that of pure jet fuel. Hiley and Pedley [69] reported that organic sulfonic acids added to distillate fuel blends containing catalytically cracked stock increase deposit 
formation, sometimes very dramatically. They also found that thiophenol is a potent sediment promoter. In a continuing study, Pedley et al. [70] found that a major source of strongly-colored sediments was the oxidation of phenalene to phenalenone, followed by the reaction of phenalenone with alkyl indoles, yielding additional products that precipitate under the influence of sulfonic acids.

Bett et al. [71] reported that the oxygen compounds found in Rundle shale oil (Queensland, Australia) were mainly phenolic in nature; alkyl-substituted phenols were the major components. Most workers $[41,43,67,68]$ agree that phenols are the most damaging of the oxygen-containing polar compounds. Hara et al. [43] proposed an oxidative coupling of phenols as the aging mechanism for SRC I and II middle distillates. Brown and Karn [41] suggested that the phenols present in coal liquids undergo a free-radical mechanism to form instability products.

It has been known that oxygen gas has a deleterious effect on fuel stability $[9,22,43,44]$. However, other investigations have shown that there are no significant differences in storing fuels with or without oxygen. Hara et al. [42] found that the viscosity of SRC II middle distillate was not siginificantly increased by bubbling with oxygen for 5 days. Dorbon et al. [64], in their study of the stability of SRC and LCO's, found that there is no difference in the trend of gum formation with or without bubbling pure oxygen during storage. The response curves of total insolubles as a function of time exhibit a period of rapid formation of total insolubles and finally a leveling off to some final value. Similar trends were obtained by White [63] when he studied the stability of 21 fuel stocks for up to 96 hours stored at 95 ${ }^{\circ} \mathrm{C}$ while oxygen is being bubbled through the sample at a rate of $3 \mathrm{~L} / \mathrm{h}$. 
The proposed mechanism in this study is similar to that suggested by Bhan et al. [66] wherein the polar and aromatic compound classes are the most active in sediment formation. As it was discussed in Section 4.6, the main reactants in gum formation were found to be polars and aromatics. In the quantification of both the aromatic and polar compounds, each was measured as one group belonging to their respective compond class. Therefore, all the multi-ring aromatics are assumed to decrease at the same rate and all polar compounds decrease at the same rate. The degradation reaction may be represented as shown in Equation 4.1.

$$
\text { Aromatics + Polars } ₹ \text { Insoluble Gum }
$$

\subsubsection{The Proposed Kinetic Model}

The kinetic model was developed to establish a relationship between gum formation and the decrease in amount of aromatic and polar compound classes for the coal hyropyrolysis liquids being studied. The model also shows the effect of temperature on the rate of gum formation.

Figure 4.11 shows that as time progresses, the concentration approaches an equilibrium value. Moreover, at each temperature the conversion levels off at a different value and does not approach $100 \%$ conversion. This indicates a reversible process. The reverse reaction is the dissolution of the precipitated gum. However, it is important to note that the process is not an equilibrium phenomenon even though the term "equilibrium" is used here. The insoluble gums that were measured are the ones that did not dissolve when washed with hexane after the filtration of the 
coal liquid sample. Apparently, there is a strict path-dependency associated with gum formation that is not a characteristic of a true equilibrium system.

\subsubsection{Developing the Kinetic Model}

From Equation 4.1, the rate equation for the forward reaction can be written as,

$$
\frac{d[G]}{d t}=k_{1}[A][P]
$$

where the $[G]=$ the concentration of insoluble gum (wt. \%), $[A]=$ the concentration of aromatic compounds (wt. \%), [P] = the concentration of polar compounds (wt. \%), and, $k_{1}=$ rate constant for the forward reaction. And the rate equation for the backward reaction can be written as,

$$
-\frac{d[G]}{d t}=k_{2}[G]
$$

where $\mathrm{k}_{2}=$ rate constant for the backward reaction .

At any time, $t$, the overall rate expression for the degradation reaction can be written as follows,

$$
\frac{d[G]_{t}}{d t}=k_{1}[A]_{t}[P]_{t}-k_{2}[G]_{t}
$$

Since the concentrations of the aromatic and polar compounds were measured by ELSD, only the components with boiling point greater than $315^{\circ} \mathrm{C}$ were reported. 
Hence, the concentrations of the aromatic and polar compounds were expressed in terms of the gum concentration. From a simple material balance, the concentration of aromatic compounds at any time, $[A]_{t}$, can be written as,

$$
[A]_{t}=[A]_{o}-[G]_{t}
$$

where $[A]_{0}=$ initial concentration of aromatic compounds. Similarly, the concentration of polar compounds at any time, $[\mathrm{P}]_{\mathrm{t}}$, can be written as,

$$
[P]_{t}=[P]_{o}-[G]_{t}
$$

where $[\mathrm{P}]_{0}=$ initial concentration of polar compounds.

Substituting Equations 4.5 and 4.6 into Equation 4.4 gives,

$$
\frac{d[G]_{t}}{d t}=k_{1}\left([A]_{O}-[G]_{t}\right)\left([P]_{O}-[G]_{t}\right)-k_{2}[G]_{t}
$$

where $[G]_{t}=0$ at $t=0$, and $[G]_{t}=[G]_{t}$ at $t=t$. Rearranging Equation 4.7 and integrating from 0 to $t$ gives,

$$
\int_{o}^{[G]_{t}} \frac{d[G]_{t}}{k_{1}\left([A]_{O}-[G]_{t}\right)\left([P]_{O}-[G]_{t}\right)-k_{2}[G]_{t}}=\int_{o}^{t} d t
$$

Integration of Equation 4.8 was performed by an integration software (www.integrals.com). Equation 4.9 shows the result of the integration of Equation 4.8. The $[G]_{t}$ terms cannot be completely isolated from the $k_{1}$ and $k_{2}$ terms. 


$$
\frac{-2 \operatorname{Arctan}\left[\frac{\left(\sqrt{k_{1}}\left([A]_{o}+[P]_{o}-2[G]_{t}\right)\right.}{\sqrt{-[A]_{o}^{2} k_{1}-4 k_{2}[G]_{t}+2[A]_{o} k_{1}[P]_{o}-k_{1}[P]_{o}^{2}}}\right]}{\sqrt{k_{1} \sqrt{-[A]_{o}^{2} k_{1}-4 k_{2}[G]_{t}+2[A]_{o} k_{1}[P]_{o}-k_{1}[P]_{o}^{2}}}}+\frac{2 \operatorname{Arctan}\left[\frac{\sqrt{k_{1}}\left([A]_{o}+[P]_{o}\right)}{\sqrt{-k_{1}}\left([A]_{o}-[P]_{0}\right)}\right]}{\sqrt{k_{1}} \sqrt{-k_{1}}\left([A]_{o}-[P]_{o}\right)}=t
$$

Thus, Equation 4.7 was solved by iteration using the SigmaPlot Software (Version 3.0). The kinetic data (time, $t$, gum concentration, [G], and change in gum concentration, $\mathrm{d}[\mathrm{G}] / \mathrm{dt}$ ) at each temperature (Appendix 4) were fitted in Equation 4.7. The absolute amount of the unaccounted components of the coal liquid sample with boiling point less than $315^{\circ} \mathrm{C}$ was estimated by taking the difference between $100 \%$ and the total weight percent of the saturate, aromatic and polar compounds. The result was divided into three and each was added to the initial concentrations of the saturate, the aromatic and the polar compounds. On the average, only $3 \%$ was added to each compound class indicating a very small fraction of unaccounted material.

SigmaPlot Software was used to solve for $k_{1}$ and $k_{2}$. SigmaPlot and SigmaStat nonlinear regression uses the Marquardt-Levenberg algorithm to find the coefficients and parameters of the independent variables that give the "best fit" between the equation and the data. This algorithm seeks the values of the parameters that minimize the sum of the squared differences between the observed and predicted values of the dependent variable.

This process is iterative-SigmaPlot and SigmaStat begin with a "guess" of the parameters, check to see how well the equation fits, then continue to make better guesses until the differences between the residual sum of squares no longer decreases significantly. This condition is known as convergence. 
The reaction rate constants obtained from Equation 4.7 at different storage temperatures are shown in Table 4.10. The forward reaction rate constant is more sensitive to storage temperature than the backward reaction rate constant. The $\mathrm{R}^{2}$ values in Table 4.10 show that it is a good fit.

Table 4.10. Reaction Rate Constants Obtained from Equation 4.7 at Different Storage Temperatures

\begin{tabular}{|c|c|c|c|}
\hline Storage Temperature $\left({ }^{\circ} \mathrm{C}\right)$ & $\mathrm{k}_{1}\left(\mathrm{hr}^{-1}\right)$ & $\mathrm{k}_{2}\left(\mathrm{hr}^{-1}\right)$ & $\mathrm{R}^{2}$ \\
\hline 2 & $3.935 \times 10^{-4}$ & $2.800 \times 10^{-2}$ & 0.999 \\
\hline 21 & $1.141 \times 10^{-3}$ & $3.148 \times 10^{-2}$ & 0.996 \\
\hline 32 & $2.132 \times 10^{-3}$ & $4.224 \times 10^{-2}$ & 0.994 \\
\hline
\end{tabular}

Figures 4.51 - 4.53 show the comparison between the $d[G] / d t$ from data versus the $d[G] / d t$ from the model for all storage temperatures. The fact that the plot of the data points versus the points predicted by the model scatter around the diagonal line indicates that the model is very good. Figure 4.54 shows the comparison of the actual data and the data obtained from the model.

The Arrhenius equation (Equation 4.10) describes the temperature dependence of $k_{1}$ and $k_{2}$,

$$
k=k_{o} e^{-\frac{E}{R T}}
$$

where $\mathrm{k}=$ reaction rate constant (forward or backward), $k_{o}=$ frequency factor, $E=$ activation energy, $\mathrm{R}$ = gas constant, and $\mathrm{T}$ = absolute temperature. Equation 4.10 can be rearranged into Equation 4.11. 


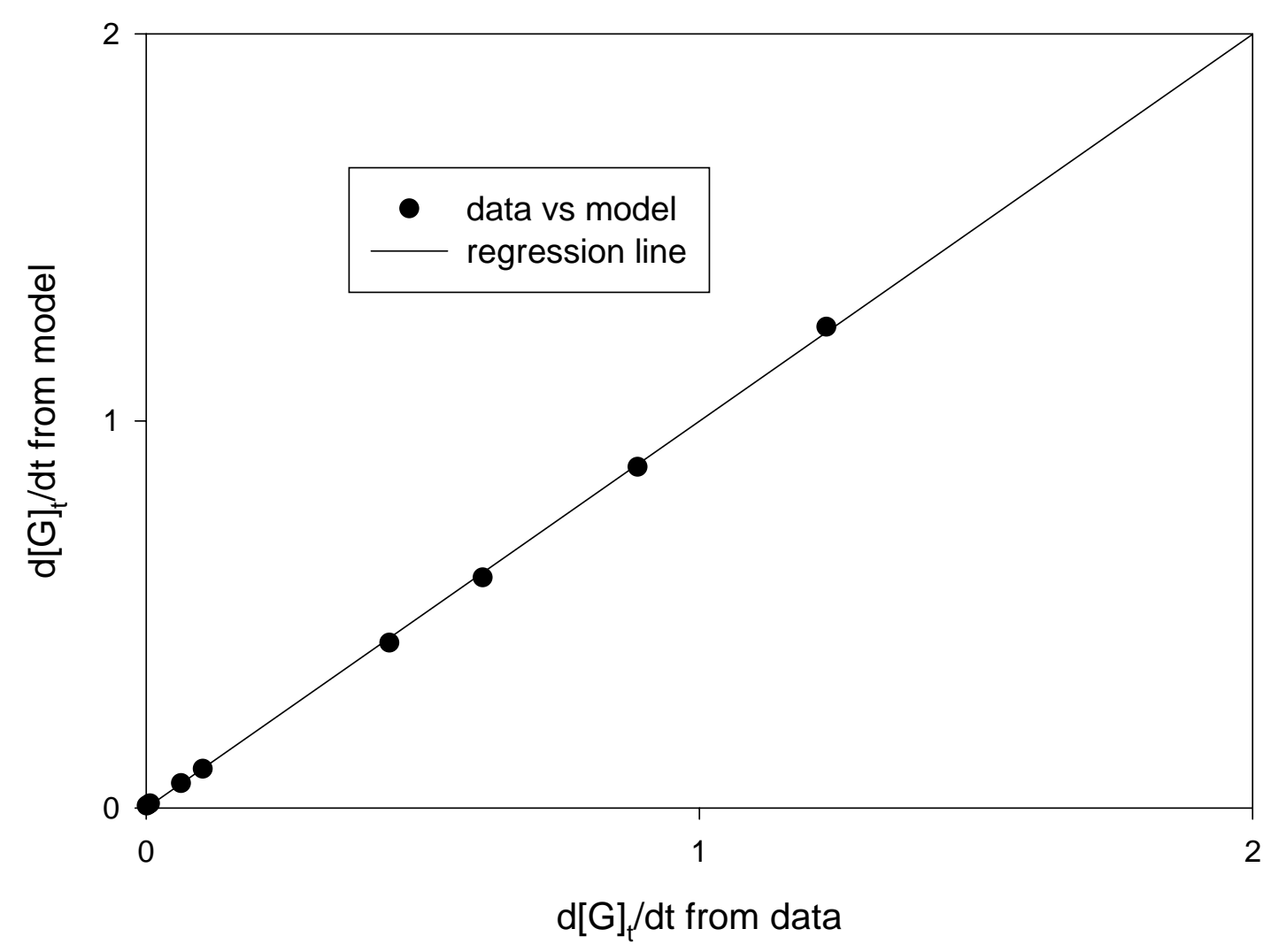

Figure 4.51 - Plot of the Rate of Gum Formation from Experimental Data versus Kinetic Model at $\mathrm{T}=2^{\circ} \mathrm{C}$ 


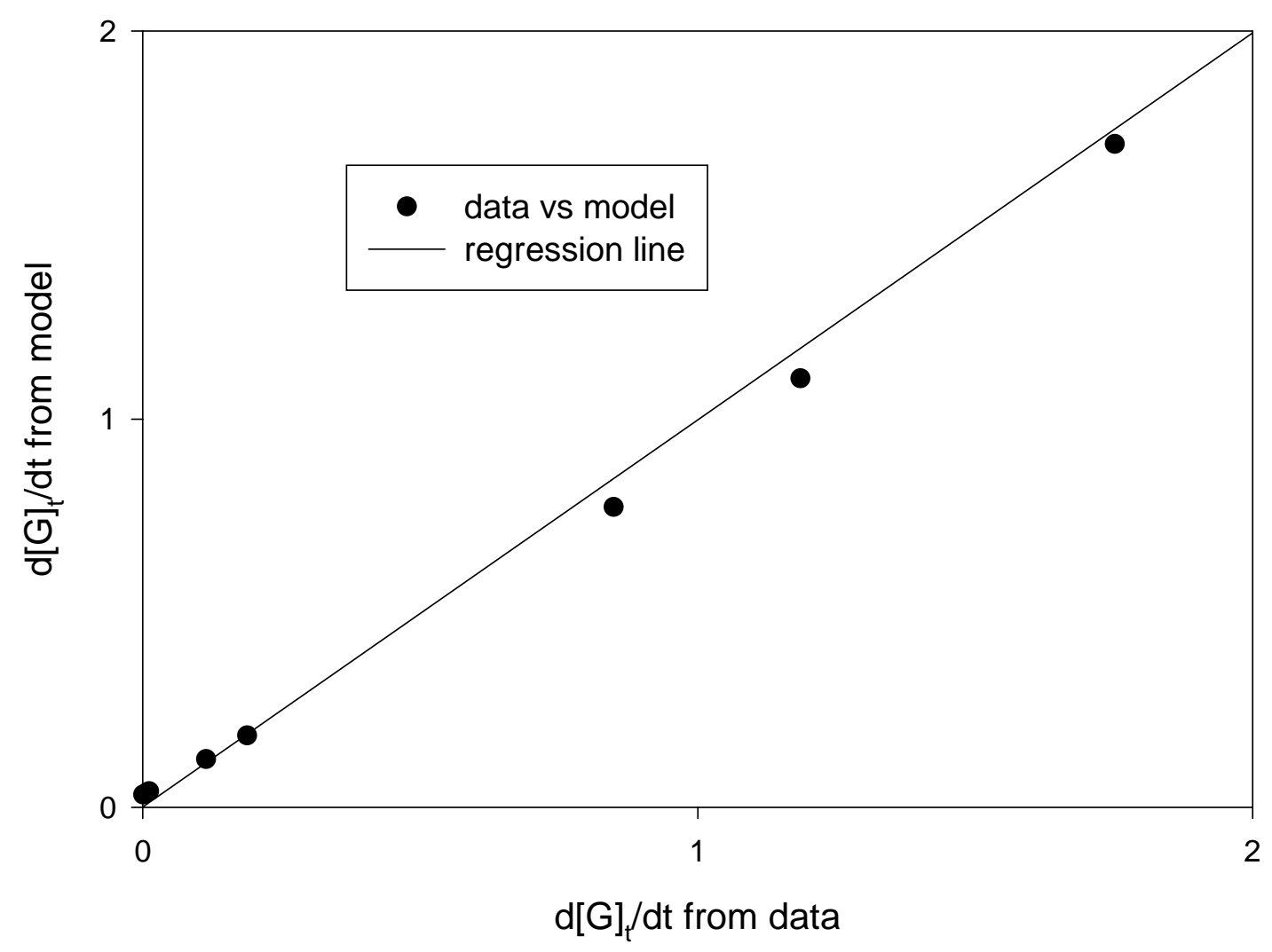

Figure 4.52 - Plot of the Rate of Gum Formation from Experimental Data versus Kinetic Model at $\mathrm{T}=21^{\circ} \mathrm{C}$ 


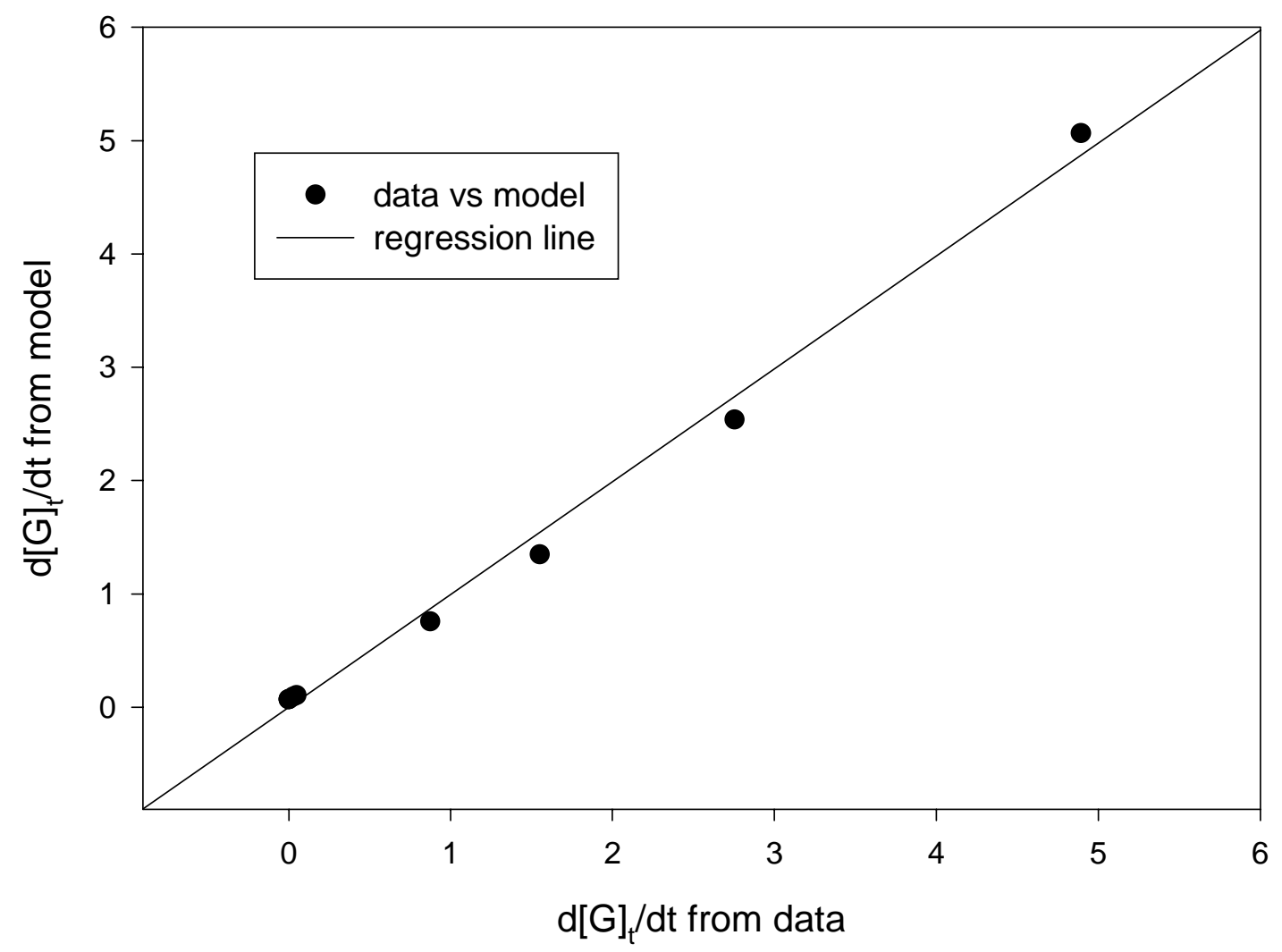

Figure 4.53 - Plot of the Rate of Gum Formation from Experimental Data versus Kinetic Model at $\mathrm{T}=32{ }^{\circ} \mathrm{C}$ 


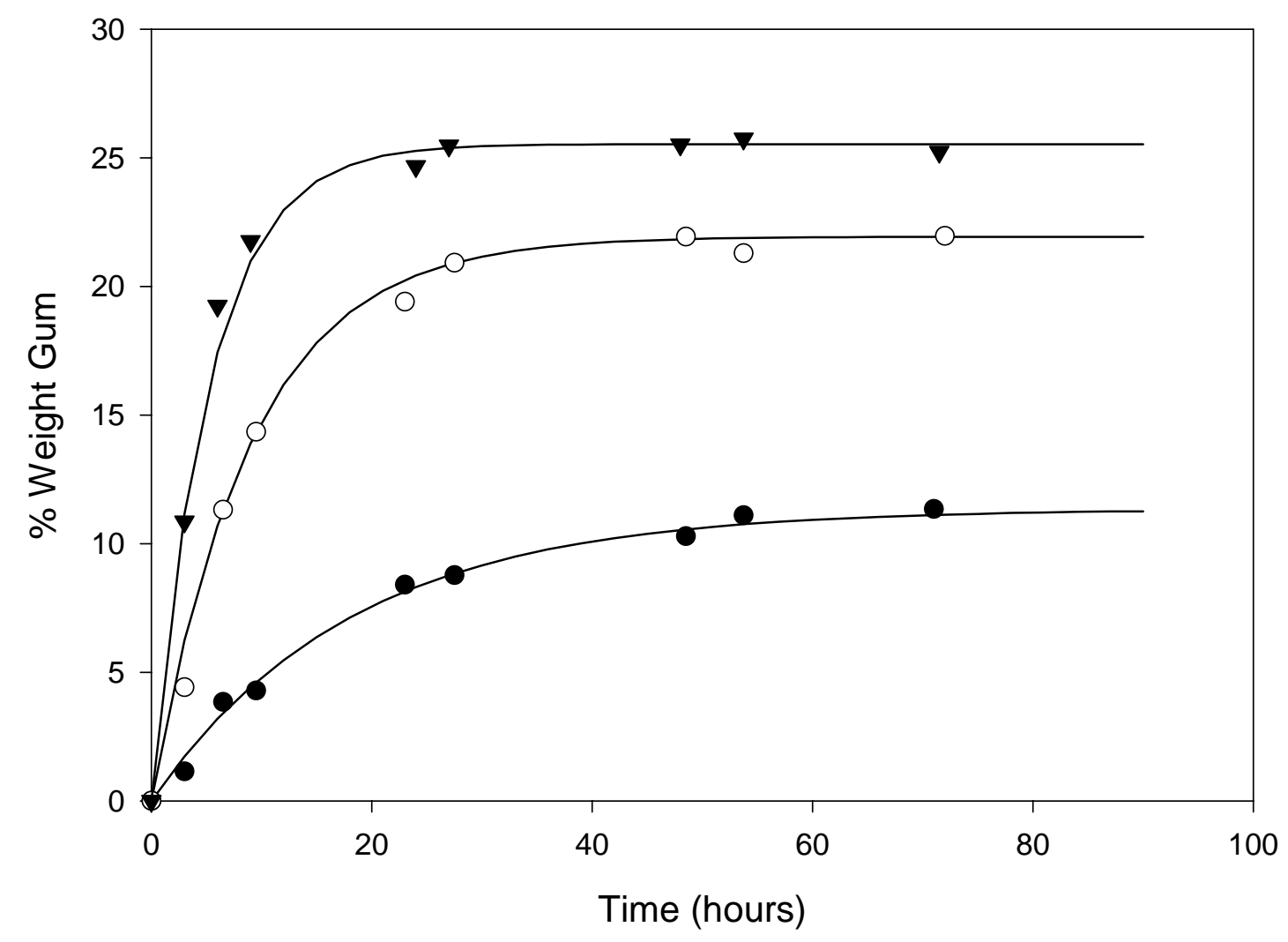

Figure 4.54 - Plot of Gum Formation from Experimental Data versus Data from the Model at 2,21 , and $32{ }^{\circ} \mathrm{C}$

$$
\begin{aligned}
& -\quad 2{ }^{\circ} \mathrm{C} \text { from model } \\
& -\quad 2{ }^{\circ} \mathrm{C} \text { from data } \\
& -\quad 21^{\circ} \mathrm{C} \text { from model } \\
& \mathrm{O} 21^{\circ} \mathrm{C} \text { from data } \\
& \hline \quad 32{ }^{\circ} \mathrm{C} \text { from model } \\
& \nabla \quad 32{ }^{\circ} \mathrm{C} \text { from data }
\end{aligned}
$$




$$
\ln k=\ln k_{o}-\frac{E}{R}\left(\frac{1}{T}\right)
$$

Figure 4.55 shows the Arrhenius plot of the rate of gum formation of coal pyrolysis liquids. The data for the Arrhenius plot are presented in Appendix 5. These data are fitted to a straight line by performing a linear regression. The Arrhenius plot for the forward reaction yields a straight line with a slope of $-4703 \mathrm{~K}$ and $R^{2}$ value of 0.999 , which corresponds to an activation energy of $9.3 \mathrm{kcal} / \mathrm{mol}$. The Arrhenius plot for the backward reaction results in a straight line with a slope of - $1064 \mathrm{~K}$ and $R^{2}$ value of 0.939 , which corresponds to an activation energy of 2.1 $\mathrm{kcal} / \mathrm{mol}$. The activation energy of the backward reaction is found to be smaller than that for the forward reaction. This suggests that the backward reaction is less temperature sensitive than the forward reaction as observed above. The low value of activation energy suggests that the amount of energy that must be overcome to form gum is low. This supports the observation that gum readily forms within a few hours into the storage.

At equilibrium, $\mathrm{d}[\mathrm{G}]_{\alpha} / \mathrm{dt}=0$ and Equation 4.7 becomes,

$$
k_{1}\left([A]_{\circ}-[G]_{\infty}\right)\left([P]_{\circ}-[G]_{\infty}\right)=k_{2}[G]_{\infty}
$$

The equilibrium constant, $\mathrm{K}$, can be expressed as,

$$
K=\frac{k_{1}}{k_{2}}=\frac{[G]_{\infty}}{\left([A]_{\circ}-[G]_{\infty}\right)\left([P]_{\circ}-[G]_{\infty}\right)}
$$




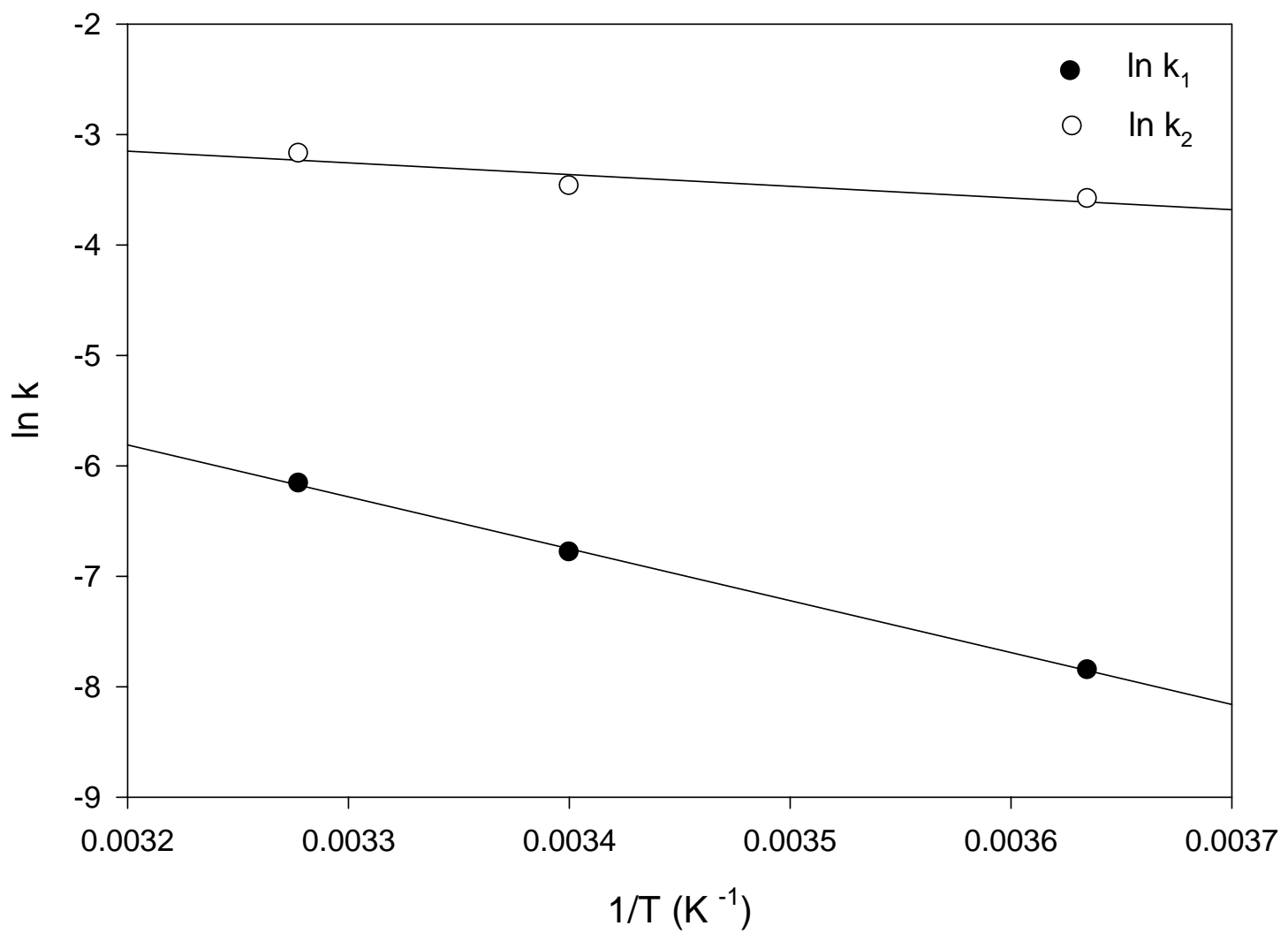

Figure 4.55 - Arrhenius Plots for the Forward and Backward Reactions of Coal Pyrolysis Liquids 
Table 4.11 shows the values for the equilibrium constants at different storage temperatures. The results show that $\mathrm{K}$ increases as the storage temperature increases. This suggests that the reaction is endothermic.

Table 4.11. Equilibrium Constants Obtained from Equation 4.13 at Different Storage Temperatures

\begin{tabular}{|c|c|}
\hline Storage Temperature $\left({ }^{\circ} \mathrm{C}\right)$ & Equilibrium Constant, $\mathrm{K}$ \\
\hline 2 & 0.014 \\
\hline 21 & 0.036 \\
\hline 32 & 0.050 \\
\hline
\end{tabular}




\subsection{Summary and Conclusions}

A direct method for determining the molecular weight distribution of coal and petroleum-derived heavy oils by using the evaporative light-scattering detector has been developed. A series of alkane and aromatic standards with increasing molecular weight were tested. For the alkane series, when the detector temperature is set at $40,80,115$, and $150^{\circ} \mathrm{C}$, sample components with $\mathrm{MW}$ greater than $254,324,394$, and 464 are detected, respectively. For the aromatic series, when the detector temperature is set at $40,80,115$, and $150{ }^{\circ} \mathrm{C}$, sample components with MW greater than 193, 215, 226, and 278 are detected, respectively. The average MW detection limits of the alkane and aromatic series were taken for all detector temperatures. Different mixtures of alkane and aromatic standards were tested and results show that the average MW detection limits chosen were incorrect. There are significant differences in the MW detection limits between the alkane and aromatic series. This difference increases as the detector temperature increases.

However, the alkane MW detections limits can be used to measure the molecular weight distribution of samples which consist of mainly saturates, like petroleum or wax. Similarly, the aromatic MW detection limits can be used to measure the molecular weight distribution of samples which consist mainly of polyaromatic hydrocarbons, like coal-tar pitch or polycyclic aromatic compounds. The advantages for using this method are: 1) the sample size for the determination is small; 2) the separation and adsorption problems are avoided because no columns are used in the analysis; and, 3) the detection limits are linear which will 
permit determination of MW detection limits at temperatures not investigated.

The aging of coal liquids has been studied. Coal liquid products were placed in preweighed sample vials and stored under air and fluorescent light at different storage temperatures $\left(2,21,32^{\circ} \mathrm{C}\right)$. The weight of gum formed was determined at specified sampling times. The HPLC-Hydrocarbon Compound Class Analysis [49] was used to monitor the changes in hydrocarbon class composition with storage time. The changes in boiling point distribution were also determined using the Simulated Distillation by ELSD [48].

All coal liquid samples became darker in color with time. The gum deposited on the walls and at the bottom of the sample vials became thicker in appearance as time progressed. Results show that the rate of gum formation is fastest at the highest storage temperature. The gum formed rapidly at the early stage of storage and then remained fairly constant with time. At each temperature, the final gum formation levelled off at a different value and not at $100 \%$ conversion. This phenomenon is consistent with a reversible reaction. The gum that is formed approaches an equilibrium value. However, it is important to note that the process is not an equilibrium phenomenon even though the term "equilibrium" is used here. Apparently, there is a strict path-dependency associated with gum formation that is not a characteristic of a true equilibrium system.

It was found that the saturate compound class remained constant with time at different storage temperatures. The increase in gum formation with time was correlated with the corresponding decrease in aromatic and polar compound classes. It was hypothesized that the aromatic compounds may be reacting with the 
polar compounds to produce the insoluble gum. This hypothesis was put to the test when fresh coal liquid samples were passed through a solid phase extraction tube containing hydroxyl groups that traps polar compounds. The aromatic and saturate compound classes passed through the extraction tube while the polar compounds were retained in the tube. The polar compounds were eluted using methylene chloride. No gum formation was observed for either the polar compounds or the aromatic-saturate mixture. Results from the Hydrocarbon Compound Class Analysis and Simulated Distillation Method show no signs of changes in their chemical composition and boiling point distribution. The polar compounds and the aromatic-saturate mixture were mixed together once again and gum formation was observed after four days. Based on these results, the main reactants in gum formation in coal liquids are the aromatic and polar compound classes.

Results from GC/MS show that the polar compounds consist mainly of phenols. This finding was confirmed by FTIR results which show that the polar compounds contain -OH groups. Hara et al. [43] also identified the presence of phenols in their study of the aging characteristics of SRC I and SRC II middle distillates. Brown and Karn [41] found an appreciable concentration of phenols in coal liquids. Ueda et al. [67] detected the presence of phenols when they characterized the chemical composition of coal-derived liquids using field ionization MS.

The effect of air on the stability of coal liquids was determined by storing coal liquid samples in the absence of air. Results show that the saturate compound class remained constant with time and is not affected by the absence of air. 
However, the gum formation was affected by the absence of air. There was a slower but steady increase in gum formation compared to the samples stored with air which have an abrupt increase in gum formation early into the storage period. The amount of gum formed is also significantly lower compared to those samples stored with air. Both the aromatic and polar compounds decreased gradually in the absence of air. The rate of decrease for both compound classes was significantly slower compared to those samples stored in air. It is then concluded that the absence of air does not prevent the formation gum; instead, it slows down the rate of gum formation.

The slow increase in gum formation and the slow decrease in aromatic and polar compounds in the absence of air suggests that the oxygen in air plays an important role in gum formation. However, other studies showed that even with continuous supply of oxygen during storage, the rate of degradation levels off [ $[42$, 64]. Other degradation reactions in the absence of oxygen can also occur as shown in the Figure 4.29 where gum formed at a steady but slow rate. Acidic components in the sample mixture may have catalyzed the degradation reaction [65]. Thus, it is possible that the oxygen in air only plays a role in the initiation or acts as a catalyst of the degradation reaction. The effect of oxygen was not investigated in this study. This would be an interesting topic for future research.

The kinetic model was developed to establish a relationship between gum formation and the decrease in amount of aromatic and polar compound classes for the coal hyropyrolysis liquids being studied. The proposed degradation reaction for coal liquids is: 


$$
\text { Aromatics + Polars } ₹ \text { Insoluble Gum }
$$

Equation 4.1 was chosen for the following reasons:

1) There is a strong evidence that there is a reaction between the polars and aromatics.

2) The insoluble gums that are formed are the ones that did not dissolve when washed several times with hexane after filtration of the coal liquid sample. These insoluble gums were measured to obtain the response curves of gum formation as a function of time.

3) The kinetic data have a characteristic feature of a reversible process. The reverse reaction is the dissolution of the precipitated gum. However, it is important to note that the process is not an equilibrium phenomenon. Apparently, there is a strict path-dependency associated with gum formation that is not a characteristic of a true equilibrium system.

The reaction rate constants for the forward reaction, $\mathrm{k}_{1}\left(\mathrm{hr}^{-1}\right)$, for storage temperatures 2,21 and $32{ }^{\circ} \mathrm{C}$ are $3.935 \times 10^{-4}, 1.141 \times 10^{-3}$, and $2.132 \times 10^{-2}$, respectively. The reaction rate constants for the backward reaction, $\mathrm{k}_{2}\left(\mathrm{hr}^{-1}\right)$, for storage temperatures 2,21 and $32^{\circ} \mathrm{C}$ are $2.800 \times 10^{-2}, 3.148 \times 10^{-2}$, and $4.224 \times 10^{-2}$, respectively. Plots of experimental data points vs data points from the kinetic model for storage temperatures 2,21 and $32{ }^{\circ} \mathrm{C}$ show linearity with $R^{2}$ values of 0.999 , 0.996, and 0.994 , respectively. This suggests that the model is very good.

Arrhenius plot for the forward reaction yields an activation energy of 9.3 $\mathrm{kcal} / \mathrm{mol}$. The Arrhenius plot for the backward reaction results in an activation 
energy of $2.1 \mathrm{kcal} / \mathrm{mol}$. The activation energy of the backward reaction is found to be smaller than that for the forward reaction. This suggests that the backward reaction is less temperature sensitive than the forward reaction.

\subsection{Future Work}

Research in the following areas would provide good follow-up for the work that was presented in this dissertation:

1) Molecular Weight Distribution

Very little work has been done on the determination of molecular weight distribution for coal and petroleum-derived heavy oils. It would be very interesting to research a way to refine the molecular detection limits for mixtures that contain saturates and aromatic compounds. The efficiency of the method developed in this study can be tested further by using it on different types of heavy oils and comparing the results with the existing and commonly used methods for determining the molecular weight distribution.

2) Gum Formation

The changes in gum composition with storage time would give information of what is being formed during the storage period. This would provide an idea of what types of compounds are incorporated into the gum. 


\section{3) The Effect of Oxygen}

Although it is well known that oxygen has a detrimental effect on the stability of fuels, it would be interesting to know the effect of bubbling oxygen gas into the coal liquid sample. Monitoring the gum composition during storage time would indicate whether oxygen is participating in gum formation.

4) Kinetic Model

Incorporation of oxygen during storage would improve the kinetic model if it can be shown that oxygen participates in gum formation. This could be proved by using isotopically labeled oxygen and analyzing the products by GC/MS. An ultimate analysis will also check what happens to oxygen and nitrogen during the reaction. 


\subsection{REFERENCES}

1. Fathoni, A. Z. And Batts, B. D., A Literature Review of Fuel Stability Studies with a Particular Emphasis on Shale Oil, Energy and Fuels, 1992, 6(6), 681.

2. Frankenfeld, J. W. and Taylor, W. F., Storage Stability of Synfuels from Oil Shale---General Features of Sediment Formation in Model Fuel Sytems, Ind. Eng. Chem. Prod. Res. Dev., 1983, 22, 608.

3. Skidmore, D. R., Fundamentals of Coal Liquefaction, Coal Research Bureau, Report No. 145, 1977.

4. National Reasearch Council, Committee on Processing and Utilization of Fossil Fuels Ad Hoc Panel on Liquefaction of Coal, Assessment of Technology for the Liquefaction of Coal: Summary (Washington, D.C.: National Academy of Sciences, 1977).

5. Fischer, R. H. and Hildebrand, R. E., Refining of Coal Derived Syncrudes, ACS Symposium, 1981, 156, 251.

6. Brinkman, D.W., Bowden, J. N., Frankenfeld, J. and Taylor, B., Synfuel Stability: Degradation Mechanisms and Actual Findings, ACS Symposium Series, 1981, 163 (20), 297.

7. Frankenfeld, J.W., Taylor, W. F. and Brinkman, D.W., Storage Stability of Synfuels from Oil Shale---Effects of Nitrogen Compound Type and the Influence of Other Nonhydrocarbon on Sediment Formation in Model Fuel Systems, Ind. Eng. Chem. Prod. Res. Dev., 1983, 22, 615.

8. Frankenfeld, J.W., Taylor, W. F. and Brinkman, D.W., Storage Stability of Synfuels from Oil Shale---Studies with Actual Shale-Derived Middle Distillates, Ind. Eng. Chem. Prod. Res. Dev., 1983, 22, 622.

9. Batts, B. D. And Fathoni, A. Z., A Literature Review on Fuel Stability Studies with Particular Empahasis on Diesel Oil, Energy and Fuels, 1991, 5, 2.

10. Mushrush, G. W., Beal, E. J., Watkins, J. M., Morris, R.E., and Hardy, D.R., Interactive Chemical Effects and Instability of Shale Derived Middle Distilallate Fuels, Oil Shale Symp. Proc. , 1989, 22nd, 172. 
11. White, E.W., Storage Stability of Distillate Fuels for Ships, Manual on Requirements, Handling, and Quality Control of Gas Turbine Fuels, ASTM STP, 1973, 531, 143.

12. Bowden, J.N. and Brinkman, D.W., Stability of Alternate Fuels, Hydrocarbon Process, 1980, 59(7), 77.

13. Furcola, N.C., Ed., Standard Test Method for Existent Gum in Fuels by Jet Evaporation, Annual Book of ASTM Standards, ASTM Philadelphia, PA, 1995, 150.

14. Furcola, N.C., Ed., Standard Method for ASTM Color of Petroleum

Products, Annual Book of ASTM Standards, ASTM Philadelphia, PA, 1995, 520.

15. Furcola, N.C., Ed., Standard Method for Distillate Fuel Storage stability at $43^{\circ} \mathrm{C}$, Annual Book of ASTM Standards, ASTM Philadelphia, PA, 1995, 913.

16. Furcola, N.C., Ed., Standard Test Method for Oxidation Stability of Distillate Fuel Oil (accelerated method), Annual Book of ASTM Standards, ASTM Philadelphia, PA, 1995, 776.

17. Stavinoha, L.L., Westbrook,R., and Brinkman, D.W., Accelerated Stability Test Techniques for Middle Distillate Fuels, U.S. Dept of Energy Report No. DOE/BC/10043-12, 1980.

18. Goetzinger, J. W., Thompson, C.J., and Brinkman, D.W., A Review of Storage Stability Characteristics of Hydrocarbon Fuels, U.S. Department of Energy Report No. DOE/BETC/IC-83/3, 1983.

19. Bowden, J.N., Stability Survey of Hydrocarbon Fuels, Final Report, DOE/BETC/1778-4, 1979.

20. Nixon, A.C., Minor, H.B. and Calhoun, G.M., Effect of Alkyl Phenols on Storage and Manifold Stability of Gasolines, Ind. and Eng. Chem., 1956, 48, 1874.

21. Schwartz, F.G., and Ward, C.C., Fundamentals of Fuel Stability, Tech. Report No. 15, Department of Army Project 1A024401A106, Bureau of Mines, 1965. 
22. Schwartz, F.G., and Ward, C.C., Fundamentals of Fuel Stability, Tech. Report No. 17, Department of Army Project 1A024401A106, Bureau of Mines, 1966.

23. Kawahara, F.K., Composition of Gum in Cracked Naphtha, Ind. Eng. Chem. Prod. Res. Dev., 1965, 4, 7.

24. Schwartz, F.G., Allbright, C.S. and Ward, C.C., Storage Stability of Gasoline: Oven Test for Prediction of Gasoline Storage Stability, BuMines RI 7197, 1968.

25. Bassler, G.C. and Smith, J.R., Gum and Haze Formation in Motor Gasoline, Tech. Reports No. 1 to 11, Dept, of Army Ordnance Corps Contact No. DA-04-200-ORD-349, May 1955 to December 1958.

26. Schwartz, F.G., and Ward, C.C., Fundamentals of Fuel Stability, Tech. Report No. 16, Department of Army Project 1A024401A106, Bureau of Mines, 1965.

27. Ward, C.C. and Schwartz, F.G., Motor Gasoline Stability, Tech. Report No. 4, Ordnance Project TB5-0010C, Department of Army Project 5-B5101-010, Bureau of Mines, 1957.

28. Ward, C.C. and Schwartz, F.G., Motor Gasoline Stability, Tech. Report No. 5, Ordnance Project TB5-0010C, Department of Army Project 5-B5101-010, Bureau of Mines, 1958.

29. Ward, C.C. and Schwartz, F.G., Fundamentals of Fuel Stability, Tech. Report No. 6, Ordnance Project TB5-0010C, Department of Army Project 5B51-01-010, Bureau of Mines, 1958.

30. Allbright, C.S., Whisman, M.L. and Schwartz, F.G., Use of Tritium Tracer Techniques in Studies of Gasoline Storage Stability, BuMines RI 6373, 1964.

31. Christian, J.G., Chiantella, A.J., Johnson, J.E., and Carhart, H.W., The Glass Effect in Distillate Fuel Stability, Ind. and Eng. Chem., 1958, 50(8), 1153.

32. Johnson, J.E., Chiantella, A.J., and Carhart, H.W., Application of Light Scattering to Diesel Fuel Stability Problems, Ind. and Eng. Chem., 1955, 47 (6), 1226. 
33. Worstell, J.H. and Daniel, S.R., Deposit Formation in Liquid Fuels 2. The Effect of Selected Compounds on the Storage Stability of Jet A Turbine Fuel, Fuel, 1981, 60, 481.

34. Heneman, F.C., The Effects of Organosulfur Compounds Upon the Storage Stability of Jet A Fuel, Master's Thesis, Colorado School Mines, Golden, CO, August 1981.

35. Nixon, A.C., Cole, C.A., and Minor, H.B., Effect of Composition and Storage on the Properties of Jet Fuels, J. Chem. and Eng. Data, 1959, 4(2), 187.

36. Gyarth, F.W., Dunn, F.R. Jr, and Smith, A.C. Jr., One-Day Stability Test for Distillate Fuel Oils, Am. Chem. Soc., Div. Petrol. Chem., Preprints, 1958, 3(3), 135.

37. Konrad, W.A., Tutwiler, T.S. and Shipley, N.L., New Fast Test Method for Distillate Storage Stability, Petrol. Proc., 1956, 11(9), 145.

38. Loveland, J.W., Dimeler, G.R., Bostwick, L.G. and Cali, L.J., Use of Light Scattering Electron Microscopy and Polarography in Oil Stability Studies, Proc., Am. Petrol. Inst., 1960, 40, 410.

39. Milsom, D. and Rescorla, A.R., Determination of Fuel Oil Storage Stability, Am. Chem. Soc., Div. Petrol. Chem., Preprints, 1956, 1(3), 239.

40. Walker, A.C., and Stanton, J.P., Examine Fuel Oils with Microscope, Petrol. Refiner, 1954, 33(11), 187.

41. Brown, F.R. and Karn, F.S., Stability Studies of Coal-derived Liquids, Fuel, 1980, 59, 431.

42. Karn, F.S., Brown, F.R., and Sharkey, A.G. Jr., Aging, Characteristics of Coal Liquids, Am. Chem. Soc., Div. Fuel. Chem., Preprints, 1977, 22 (2), 227.

43. Hara, T. L., Jones, L., Li, N.C. and Tewari, K.C., Aging of SRC Liquids, Fuel, 1981, 60, 1143.

44. Kershaw, J.R. and Gray, D., Aging Studies of Coal Hydropyrolysis Liquids, Fuel, 1980, 59, 436. 
45. Brinkman, D.W., Synfuel Storage and Thermal Stability Test Results, Proc. Naval Air Systems Command-Naval Research Laboratory Work-shop on Basic Research Needs for Synthetic Hydrocarbon Jet Aircraft Fuels, Washington, D.C., 1978, 155.

46. Brinkman, D.W. and Bowden, J.N., Stability of Some Coal and Tar Sands Syncrude Fractions, Fuel, 1982, 61(11), 1141.

47. Wright, C.W., and Weimer, W.C., Chemical and Biological Stability of Solvent Refined Coal Liquids, Prepared for the U.S. Department of Energy under Contract DE-AC06-76RL0 1830, Richland, Washington, 1984.

48. Padlo, D.M., and Kugler, E.L., Simulated Distillation of Heavy Oils Using an Evaporative Light Scattering Detector, Energy and Fuels, 1996, 10(5), 1031.

49. Padlo, D.M., Subramanian, R.B., and Kugler, E.L., Hydrocarbon Class Analysis of Coal-Derived Liquids Using High Performance Liquid Chromatography, Fuel Processing Technology, 1996, 49, 247.

50. Hoesterey, B.L., McClennen, W.H., Wimdig, W. and Meuzelaar, H.L.C., Computer Enhanced Separation of Compound Classes in Fuel Mixtures Using a Combined LC-MS Approach, Am. Chem. Soc., 1985, 30, 85 .

51. Thomson, J.S., and Reynolds, J.W., Separation of Aromatic Hydrocarbons, Using Bonded-Phase-Charge-Transfer Liquid Chromatography, Anal. Chem, 1984, 56, 2434.

52. Lamey, S.C., Hesbach, P.A., and White, K.D., Liquid Fuel Analysis Using High Performance Liquid Chromatography and Gas ChromatographyMass Spectroscopy, Energy and Fuels, 1991, 5, 222.

53. McKinney, D.E., Clifford, D.J., Bogdan, M.R., and Hatcher, P.G., High Performance Liquid Chromatography of Coal Liquefaction Process Streams Using Normal Phase Separation with Diode Array Detection, Energy and Fuels, 1995, 9, 90.

54. Sax, N. I., Dangerous Properties of Industrial Materials 5th Edition, Litton Education Publishing, Inc, New York, New York, 1979. 
55. Hueper, W.C., Medicolegal Considerations of Occupational and Nonoccupational Environmental Cancers, in Lawyers' Medical Cyclopedia, Vol. 5B, C.J. Frankel and R.M. Patterson, (editors) The Allen Smith Co., Indianapolis, Indiana, 1972.

56. Bartle, K. D., Collin, G., Stadelhofer, J. W. And Zander, M., J. Chem. Tech. Biotechnol., 1979, 29, 531.

57. Cebolla, V. L., Memrado, L., Vela, J., Ferrrando, A. C., and Romero, C., American Chemical Society, Division of Fuel Chemistry, Preprints, 1996, 41, 1152.

58. Mulligan, M. J., Bartle, K. D., Gibson, C. Taylor, N. and Mills, D., Proc. Int. Conf. Coal Science, 1981, p.882.

59. Bartle, K. D., Taylor, N., Mulligan, M. J., Mills, D. G. and Gibson, C., Fuel, 1983, 62, 1181.

60. Coulombe, S., Journal of Chromatographic Science, 1988, 26, 1.

61. Wyatt, P. J. and Papazian, L. A., LC-GC, 1993, 11, 862.

62. Snyder, L. R., and Kirkland, J.J., Introduction to Modern Liquid Chromatography 2nd Ed., 199, New York, 483.

63. White, E. W., A Fuel Stability Study: Total Insolubles as a Function of Time, American Chemical Society, Division of Fuel Chemistry, Preprints, 1990, 35 (4), 1184.

64. Dorbon, C. B., Gaillard, J., Denis, J., Storage Stability of Fuels, Symposium on Stability and Oxidation of Middle-Distillate Fuels, Washington, D.C., August 26-31, 1990, 1125.

65. White, E. W., A Fuel Stability Study of Filterable and Adherent Insolubles as Functions of Time, 208th ACS National Meeting, Washington, D.C., August 21-26, 1994, 648.

66. Bhan, O. K., Brinkman, D. W., Green, J. B., Storage Stability of Marine Diesel Fuels, Fuel, 1987, 66, 1200.

67. Ueda, K., Matsui, H., Malhotora, R., and Nomura, M., Chemical Characterization of Coal Tar and Coal Derived Liquid Based on Their Separation by High Performance Liquid Chromatography Analyzed by FI-MS Measurements, Sekiyu Gakkaishi, 1991, 34, 53. 
68. White, C. M., Jones, L., and Li, N. C., Ageing of SRC-II Middle Distillate from Illinois No. 6 Coal, Fuel, 1983, 62, 1397.

69. Hiley, R. W., and Pedley, J.F., Storage Stability of Petroleum-Derived Diesel Fuel, Fuel, 1988, 67, 469.

70. Pedley, J. F., Hiley, R.W., and Hancock, R.A., Storage Stability of PetroleumDerived Diesel Fuel, Fuel, 1988, 67, 1124.

71. Bett, G., Harvey, T. G., Matheson, T.W., Pratt, K.C., Determination of Polar Compounds in Rundle Shale Oil, Fuel, 1983, 62, 1445. 
APPENDIX 1:

\section{PROCEDURE USED IN COAL LIQUEFACTION}




\section{Tubing Bomb Reactor}

1) Clean the reactor before using it. Brush and scrape the inside of the reactor to remove the unreacted coal that was left from the previous reaction. Blow off the remaining coal particles in the reactor with compressed air.

2) Put lubricant on the sidearms of the reactor. Screw and unscrew the caps of the sidearms several times until the lubricant is evenly distributed on the sidearms. Wipe out all excess lubricant that went inside and outside the reactor.

\section{Coal}

1) Weigh approximately 3 to 5 grams of coal and record the weight. Pour the weighed coal sample into the previously cleaned tubing reactor. Close and tighten both sidearms of the reactor.

2) Attach the reactor to a hydrogen source. Purge hydrogen gas into the reactor at least three times. Check for leaks while purging by immersing the reactor into a bucket of water. After purging, put 1100 psig hydrogen gas into the reactor. Attach and tighten the valve of the reactor.

\section{Reaction}

1) Attach the tubing reactor to the shaker system.

2) The sandbath should be preheated to $487^{\circ} \mathrm{C}$ prior to its use.

3) Immerse the reactor attached to the shaker into the sandbath and lower the temperature to $450^{\circ} \mathrm{C}$. Set the shaker to $100 \mathrm{rpm}$ and let the reaction proceed for one hour. 


\section{Extraction}

1) After the reaction, quench the reactor in cold tap water.

2) Attach the reactor to the gas collector. Slowly release the valve and let the gas flow into the flask.

3) Unscrew the caps of the sidearms and collect the product mixture into a preweighed beaker.

4) Wash the reactor with hexane solution to facilitate the collection of the product mixture. Collect all the washings with hexane

5) Transfer everything to the preweighed extraction flask with boiling chips.

6) Reflux overnight in a Soxhlet extractor.

7) Tare and weigh beaker, filter and flask.

\section{Recovery}

1) Cool and filter the extracted mixture using a vacuum filter.

2) Wash the extraction flask with hexane and filter all the washings.

3) After filtration, place the used filter in the preweighed beaker and cover it with perforated aluminum foil. Vacuum dry the filter and beaker as well as the extraction flask at $250^{\circ} \mathrm{C}$ overnight. Cool and weigh until constant weight.

3) Transfer the filtrate into a preweighed flask.

4) Evaporate the hexane from the filtrate using a rotary evaporator.

5) Collect the concentrated hexane-soluble oil for stability studies. 


\section{APPENDIX 2:}

\section{SAMPLE CALCULATION OF TOTAL CONVERSION}




\section{Sample Data (Run \#4):}

1) Weight of Coal $=3.9241$ grams

2) Weight of Residue

Weight of Extraction Flask + Residue + Boiling Chips $=171.9842$ grams

Weight of Extraction Flask + Boiling Chips $\quad=\underline{169.1529 \text { grams }}$

Weight of Residue

$=2.8313$ grams

Weight of Beaker + Residue

$=83.0325$ grams

Weight of Beaker

$=\underline{82.7564 \text { grams }}$

Weight of Residue

$=0.2761$ grams

Total Weight of Residue $=2.8313+0.2761$

$=3.1074$ grams

3) Percent Conversion (as received basis)

$\%$ Conversion $=\underline{\text { Total Coal }- \text { Total Residue }} \times 100 \%$ Total Coal

$\%$ Conversion $=\underline{3.9241-3.1074} \times 100 \%$

3.9241

$=20.81 \%$ 
APPENDIX 3:

THREE-DIMENSIONAL UV SPECTRUM PLOT OF THE AROMATIC STANDARDS USED 


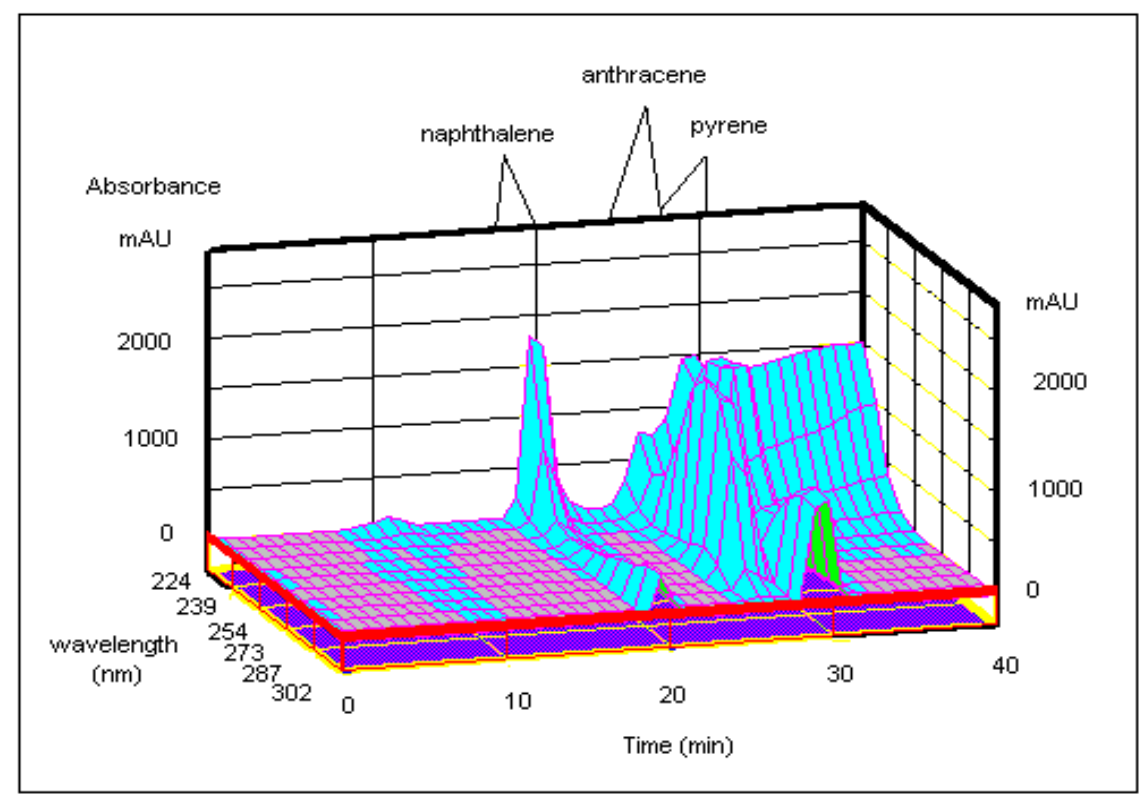

Appendix 3 - Three-Dimensional UV Spectrum Plot of Naphthalene, Anthracene, and Pyrene 
APPENDIX 4:

KINETIC DATA FOR COAL PYROLYSIS LIQUIDS 


\begin{tabular}{|c|c|c|}
\hline \multicolumn{3}{|c|}{ Kinetic Data at $\mathrm{T}=2^{\circ} \mathrm{C}$} \\
\hline Time (hours) & [G] (wt. \%) & $\underline{\mathrm{d}}[\mathrm{G}] / \mathrm{dt}$ (wt. \%/ hour) \\
\hline 0.0000 & 0.0000 & 0.6242 \\
\hline 3.0000 & 1.7264 & 0.5292 \\
\hline 6.5000 & 3.4115 & 0.4366 \\
\hline 9.5000 & 4.6190 & 0.3702 \\
\hline 23.0000 & 8.1465 & 0.1762 \\
\hline 27.5000 & 8.8489 & 0.1376 \\
\hline 48.5000 & 10.5620 & 0.0433 \\
\hline 53.7500 & 10.7596 & 0.0325 \\
\hline 71.0000 & 11.1214 & 0.0126 \\
\hline$[A]_{0}=48.37 \%$ & & \\
\hline$[P]_{0}=37.00 \%$ & & \\
\hline
\end{tabular}




\begin{tabular}{|c|c|c|}
\hline \multicolumn{3}{|c|}{ Kinetic Data at $\mathrm{T}=21^{\circ} \mathrm{C}$} \\
\hline Time (hours) & [G] (wt. \%) & $\underline{\mathrm{d}}[\mathrm{G}] / \mathrm{dt}$ (wt. \%/ hour) \\
\hline 0.0000 & 0.0000 & 2.4485 \\
\hline 3.0000 & 6.2423 & 1.7519 \\
\hline 6.5000 & 11.3182 & 1.1854 \\
\hline 9.5000 & 14.3403 & 0.8481 \\
\hline 23.0000 & 20.2554 & 0.1880 \\
\hline 27.5000 & 20.9205 & 0.1138 \\
\hline 48.5000 & 21.8421 & 0.0109 \\
\hline 53.7500 & 21.8855 & $6.0783 e-3$ \\
\hline 72.0000 & 21.9329 & $792.97 e-6$ \\
\hline \multicolumn{3}{|l|}{$[A]_{0}=47.48 \%$} \\
\hline$[\mathrm{P}]_{\mathrm{o}}=46.70 \%$ & & \\
\hline
\end{tabular}




\begin{tabular}{|c|c|c|}
\hline \multicolumn{3}{|c|}{ Kinetic Data at $\mathrm{T}=32{ }^{\circ} \mathrm{C}$} \\
\hline Time (hours) & [G] (wt. \%) & $\underline{\mathrm{d}[\mathrm{G}] / \mathrm{dt} \text { (wt. \%/ hour) }}$ \\
\hline 0.0000 & 0.0000 & 4.8935 \\
\hline 3.0000 & 11.1656 & 2.7541 \\
\hline 6.0000 & 17.4499 & 1.5501 \\
\hline 9.0000 & 20.9867 & 0.8724 \\
\hline 24.0000 & 25.2829 & 0.0493 \\
\hline 27.0000 & 25.3953 & 0.0277 \\
\hline 48.0000 & 25.5374 & $496.02 e-6$ \\
\hline 53.0000 & 25.5390 & $190.30 \mathrm{e}-6$ \\
\hline 72.0000 & 25.5400 & $4.9939 \mathrm{e}-6$ \\
\hline \multicolumn{3}{|l|}{$[A]_{0}=48.93 \%$} \\
\hline$[P]_{0}=48.56 \%$ & & \\
\hline
\end{tabular}


APPENDIX 5:

DATA FOR ARRHENIUS PLOTS 
Data for Arrhenius Plots for $k_{1}$ and $k_{2}$

Temperature $(\mathrm{K})$

275.15

294.15

305.15 $\underline{1 / T(1 / K)}$

$3.6344 \mathrm{e}-3$

$3.3996 \mathrm{e}-3$

$3.2771 \mathrm{e}-3$ $\underline{\mathrm{k}}_{1}\left(\mathrm{hr}^{-1}\right)$

$3.9350 \mathrm{e}-4$

$1.1410 \mathrm{e}-3$

$2.1320 \mathrm{e}-3$ $\underline{\mathrm{k}}_{2}\left(\mathrm{hr}^{-1}\right)$

0.0280

0.0315

0.0422 
APPENDIX 6:

GC/MS METHODS USED FOR THE ANALYSIS 


\section{[ File Control Segment}

Parameters

Conf iguration

Exit

GC Method: METHODS $\backslash$ POLAR2

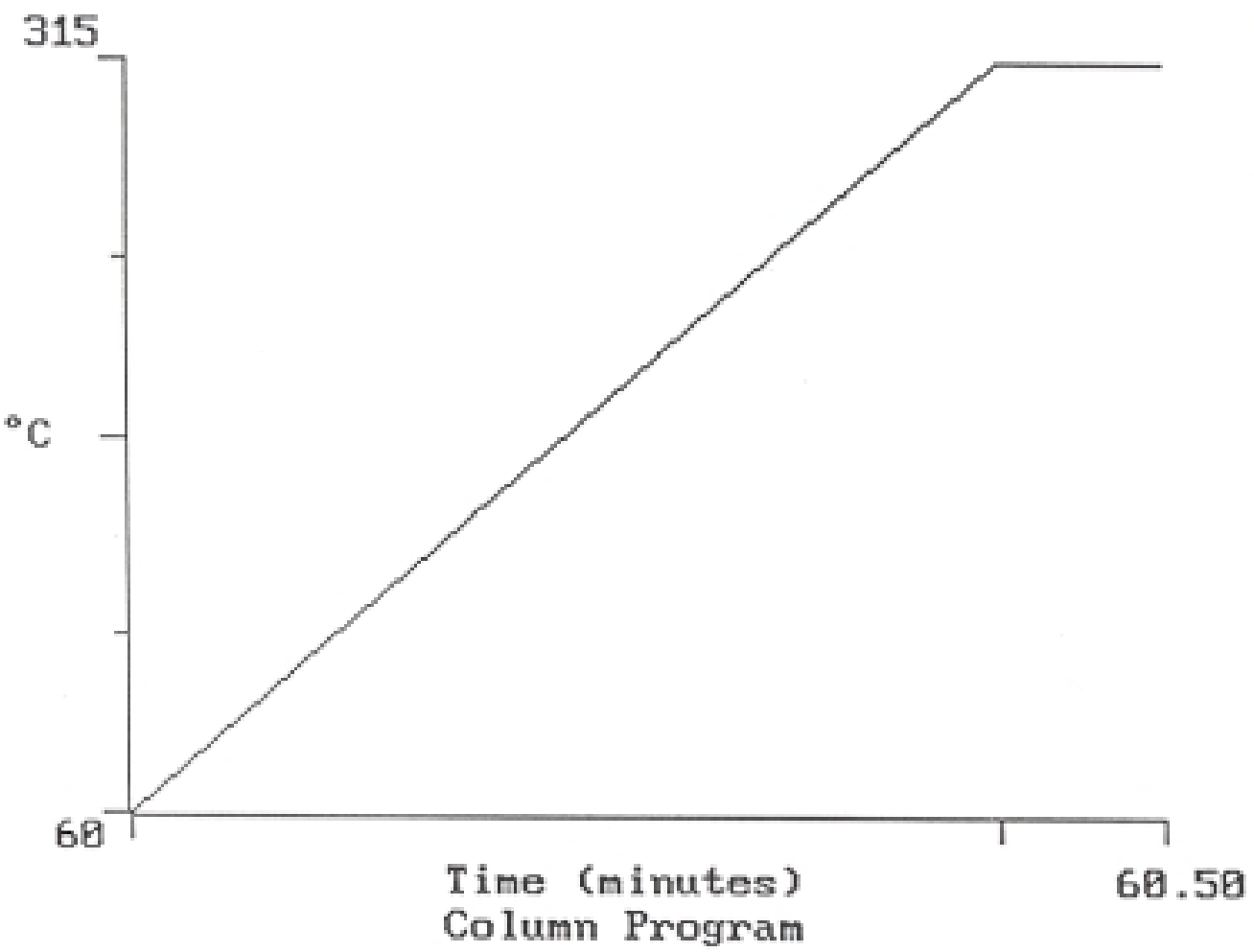

\begin{tabular}{|c|r|r|r|r|}
\hline Seg & Temp & Rate & Time & Total \\
\hline \hline 1 & 315 & 5.0 & 51.90 & 51.80 \\
\hline 2 & 315 & 8.0 & 9.50 & 60.50 \\
\hline
\end{tabular}

Column Program

Start $\quad 60{ }^{\circ} \mathrm{C}$
End $\quad 315{ }^{\circ} \mathrm{C}$
Time $51.00 \mathrm{~min}$
Rate $5.0{ }^{\circ} \mathrm{C} / \mathrm{min}$

1077

Column Injector Xfer Line Detector

Set

$60^{\circ} \mathrm{C} 250{ }^{\circ} \mathrm{C}$

$270{ }^{\circ} \mathrm{C} \quad 270{ }^{\circ} \mathrm{C}$ 
D File Segment

Bxit

Acquisition Method: METHODS $\backslash E I-P O L A R$

Current Segment: 1 of 1

Method End Time: 127.00 Segment Time: 0.00 to 127.00

\begin{tabular}{|c|c|c|c|c|c|c|}
\hline Gass Range & to & $m / z$ & Ion Mode & EI & SECI & $\mathrm{MICI}$ \\
\hline $\begin{array}{l}\text { Scan Time } \\
(2 \text { uScans })\end{array}$ & 1.000 & seconds & Ion Preparation & $\mathrm{MS} / \mathrm{MS}$ & Kone & \\
\hline & & & Ion Control & Auto & Fixed & \\
\hline Segment Length & 127.00 & minutes & Cal Gas & On & orf & \\
\hline Fil/Mul Delay & $\theta$ & minutes & & & & \\
\hline Peak Threshold & 1 & counts & & & & \\
\hline Mass Defect & 8 & $\mathrm{mu} / 180 \mathrm{u}$ & & & & \\
\hline Background Mass & 45 & $\mathrm{~m} / \mathrm{z}$ & & & & \\
\hline Tune File & \multicolumn{3}{|c|}{ 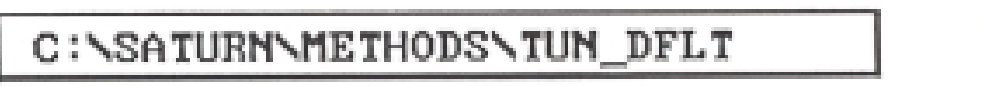 } & Use $\mathrm{L}$ & st Acti & File \\
\hline Description & DEFAULT & TUNE PARA & TERS F ILE & & Edit & Load \\
\hline
\end{tabular}

PREUIOUS SEGMENT

NEXT SEGMENT 
APPENDIX 7:

CONDITIONS USED IN HPLC ANALYSIS 
Autosampler: $\quad$ VARIAN 9095

Solvent Delivery System: VARIAN 9010

Columns: Partisil-amino-cyano (PAC), DIOL-silica (DIOL), and 2, 4dinitroanilinpropyl silica (DNAP) columns

Detectors: Evaporative Light-Scattering Detector (Varex Mark II A) Polychrome 9065 Diode Array Detector

Solvent Carrier Used: $\quad$ Pentane, Hexane, Methylene Chloride, Isopropyl Alcohol

Solvent Flowrate: $\quad 1.2 \mathrm{~mL} / \mathrm{min}$

Gas Used for ELSD: Nitrogen

ELSD Gas Flowrate: $\quad 6.3 \mathrm{~L} / \mathrm{min}$

Sample Size Injected: $\quad 80 \mu \mathrm{L}$ 


\section{BIOGRAPHICAL SKETCH}

Lawrence Norcio was born on December 25, 1968 in Los Banos, Laguna, Philippines. He is the only son of five children of Felipe and Lourdes. He completed his high school education in Letran College in 1985. He was admitted to the chemical engineering undergraduate program of the University of the Philippines (U.P.) in 1985 with a full-tuition scholarship from the Grants-in-Aid Foundation. During the first two years of his undergraduate studies, he enlisted and completed the Citizen's Military Training held at U.P. by the Philippine Army. After he received his B.S. in Chemical Engineering in 1990, he was employed by the same university as a researcher at the Institute of Biotechnology. After six months, he was hired as an instructor in chemistry at the Institute of Chemistry in U.P. He received the "Top Chemical Engineer Award" from the Professional Regulation Commission-Board of Chemical Engineers in October 15, 1991 which were given to the top 20 of 781 chemical engineers who took the chemical engineering licensure examination that year.

Lawrence was admitted to the graduate program in chemical engineering of Ohio University, Athens, Ohio in Fall 1993. He received a teaching assistant position from the Department of Chemical Engineering. He obtained his M.S. in

Chemical Engineering in August 1995. Since then, he has been a Ph.D. student at the Department of Chemical Engineering at West Virginia University, Morgantown, West Virginia. 